\title{
3. THE BENTHIC FORAMINIFERAL FAUNAS OF SITES 725, 726, AND 728 (OMAN MARGIN, NORTHWESTERN ARABIAN SEA) ${ }^{1}$
}

\author{
J. Otto R. Hermelin ${ }^{2}$
}

\begin{abstract}
The relative abundances of benthic foraminifers from the Oman margin have been analyzed from ODP Sites 725 and 726 near the upper boundary of the oxygen-minimum zone (OMZ) and 728 near the lower boundary. The relative abundance pattern of the benthic foraminiferal species in the two shallow sites show synchronous changes, which, together with variations in the faunal composition, may be attributed to changes in the location of the upper boundary of the OMZ during the last 7 million years. At the deeper site, the relative abundance pattern shows considerable variation in the faunal composition during the last 8 million years. The strong dominance of the shallow-water species Ammonia beccarii during the early Pliocene at Site 728 suggests a water depth less than $400 \mathrm{~m}$ during the early Pliocene and subsequent subsidence during the middle and late Pliocene to the present $>1400 \mathrm{~m}$ water depth.
\end{abstract}

\section{INTRODUCTION}

A major part of ODP Leg 117 was dedicated to studying the continental margin off the coast of Oman (Fig. 1A). The goal was to understand the origin and variability of organic-carbonrich sediments that result from the combined effects of monsoon-induced upwelling and the related OMZ. The main objectives of this study are to investigate the benthic foraminiferal fauna and, if possible, relate changes in the faunal assemblages to variations in the monsoonal and/or upwelling intensity.

\section{The Geological and Oceanographic History of the Oman Margin}

Much of the Oman margin is characterized by a narrow shelf bordered by an extremely steep continental slope, which is thought to be a mega-shear associated with formation of the margin (Whitmarsh, 1979; Stein and Cochran, 1985). However, the margin between Ra's Sharbithat and Ra's Madrakah has a relatively wide continental shelf (about $80 \mathrm{~km}$ wide) and a series of linear sedimentary basins along the upper continental slope. These basins lie at water depths between 500 and $1500 \mathrm{~m}$ and thus accumulate sediments coincident with the intermediate water masses of the Arabian Sea.

One of the important features of the Arabian Sea oceanography is the active oceanic upwelling and enhanced biological productivity (Currie et al., 1973; Kuz'menko, 1974; Deuser et al., 1978; Prell and Curry, 1981; Naqvi et al., 1982; Quasim, 1982). The cause of this high productivity may be attributed to an upwelling system driven by the southwest monsoon which reaches an acme in the summer months (Wyrtki, 1971; Krey and Babenerd, 1976; Prell and Streeter, 1982). Resulting divergence and Ekman transport of surface water in a southeasterly direction allows deeper, nutrient-rich, waters to well up into the euphotic zone and promote high rates of primary and secondary productivity along the coast of Oman. The underlying sediments are partially composed of microfossils and organic matter that are produced in response to this euphotic activity.

The semi-enclosed character of the northwestern Indian Ocean and the resulting sluggish circulation of highly saline North In-

\footnotetext{
${ }^{1}$ Prell, W. L., Niitsuma, N., et al., 1991. Proc. ODP, Sci. Results, 117: College Station, TX (Ocean Drilling Program).

2 Deep Sea Geology Division, University of Stockholm S-106 91, Stockholm,
}

dian Intermediate Water (NIIW) together with high rates of productivity cause extremely low oxygen concentrations of less than $0.5 \mathrm{~mL} / \mathrm{L}$ and form an oxygen minimum zone (OMZ) that extends from about 200 to $1500 \mathrm{~m}$ in the Arabian Sea (Wyrtki, 1971, 1973; Slater and Kroopnick, 1984). The most severe depletion of oxygen occur in the northern part of the Owen Basin (Price and Shimmield, 1987; Hermelin and Shimmield, 1990).

\section{Previous Work}

The Recent benthic foraminiferal fauna in this area has been studied by Hermelin and Shimmield (1990). They found that the benthic foraminiferal fauna was strongly related to the geochemistry of the sediment and the depositional environment. In short, their results revealed six different foraminiferal assemblages, each related to a specific depositional environment.

1. The species Bolivina pygmaea, Bulimina sp. 1, and Lenticulina iota are good indicators for organic-rich sediment deposited on the shelf in the upper bathyal zone within the upper part of the OMZ.

2. The benthic foraminiferal fauna in the lower part of the OMZ (middle bathyal zone), where the carbonate content is high, is dominated by the species Ehrenbergina trigona, Hyalinea balthica, Tritaxia sp. 1, and Uvigerina peregrina.

3. Just below the $\mathrm{OMZ}$, in the lower bathyal zone, the fauna is dominated by Bulimina aculeata and Uvigerina hispida.

4. The depositional area furthest from the continent on the ridges and on the outer Indus fan (abyssal zone) is dominated by Bulimina aculeata, Oridorsalis umbonatus, and Uvigerina spinicostata.

5. The sediment deposited closer to the continent at the base of the continental slope and in the Owen Basin is characterized by a high content of hydrogenous metals in the fine-grained clay, and the benthic foraminiferal fauna is dominated by the arenaceous species Reophax bilocularis and Reophax dentaliniformis.

6. The Gulf of Oman, characterized by a largely terrigenous sediment type with (presumably) high sediment accumulation rates, exhibits a different faunal composition with the arenaceous form Cribrostomides wiesneri as the dominant species. The species Reophax bilocularis is also an important species in this depositional environment.

Other studies of Recent and late Neogene benthic foraminifers of this area are scarce, although some have been carried out 
in other parts of the Indian Ocean: Ninetyeast Ridge (Boltovskoy, 1978); southeast Indian Ocean (Corliss, 1979a, b).

\section{MATERIAL AND METHODS}

\section{Locations}

A series of eight sites was drilled in the northwestern Arabian Sea on the continental margin off Oman. Three of these sites $(725,726$, and 728$)$ are discussed in this report.

Site 725 is located in $311 \mathrm{~m}$ of water at $18^{\circ} 29.200^{\prime} \mathrm{N}$ and $57^{\circ} 42.030^{\prime} \mathrm{E}$ (Fig. 1B). It is near the upper limit of the OMZ, in a transitional zone between the continental shelf and the landward edge of a slope basin (Fig. 2). Because of the location of this site at the shelf edge, winnowing of fine sediment can be expected.

Site 726 is located in $330 \mathrm{~m}$ of water at $17^{\circ} 48.945^{\prime} \mathrm{N}$ and $57^{\circ} 22.290^{\prime} \mathrm{E}$ on the continental shelf of Oman (Fig. 1B). It is positioned near the shelf-edge transition on the landward flank of a slope basin and, like Site 725, close to the upper limit of the OMZ (Fig. 2).

Site 728 is located in $1436 \mathrm{~m}$ of water at $17^{\circ} 40.790^{\prime} \mathrm{N}$ and $57^{\circ} 49.553^{\prime} \mathrm{E}$ on the continental margin of Oman (Fig. 1B). That water depth correspond in this area to the lower part of the pronounced mid-water OMZ. The site is located in a deep slope basin formed by a narrow half-graben in what is presumed to be ophiolitic basement (Fig. 2).

\section{Benthic Foraminifers}

Approximately $10 \mathrm{~cm}^{3}$ of sediment was washed through a 63 $\mu \mathrm{m}$ sieve and the larger fraction was dried. The larger fraction was thereafter dry-sieved through a $125 \mu \mathrm{m}$ sieve, and the benthic foraminifers were picked from the larger fraction. The counting procedure consisted of splitting the $>125 \mu \mathrm{m}$ fraction into an aliquot containing 300-400 specimens. Chang (1967) showed that identification of 300 randomly selected specimens from a larger assemblage provides a valid data base for statistical analysis, and that the results are not significantly improved by examining greater number of specimens. The specimens were then identified to species level, counted, and statistically analyzed. Note that the relative abundance calculated for Sites 725 and 726 is given in 5\%-intervals (Table 1 and 2), whereas it is given to one decimal for Site 728 (Table 3). All species recognized are presented in the "Systematic Paleontology" section and the most important species are figured on Plates 1-3. Additionally the number of specimens per cubic centimeter was calculated.

\section{Age Models}

The age models for Sites 725,726 , and 728 used here are based on biostratigraphic and paleomagnetic information from the Initial Reports of ODP Leg 117 (Prell, Niitsuma, et al., 1989). The "absolute" ages for nannofossil, radiolarian, planktonic foraminiferal datum levels, magnetostratigraphic boundaries, and zonal boundaries are derived from various sources. The datum markers used, their location in Sites 725, 726, and 728 , and their age assignments with references are presented in Table 4. In the Initial Reports of ODP Leg 117 (Prell, Niitsuma, et al., 1989), additional biostratigraphic datum levels were recognized but these were excluded here due to their unreliability as "absolute" age markers. Therefore, the age models used here are slightly different from those initially presented (Prell, Niitsuma, et al., 1989).

\section{Sediment Accumulation Rates}

The rate of sedimentation at Site 725 is calculated to be 11.1 $\mathrm{cm} / \mathrm{k}$.y. based on interpolations between the sediment-water interface and LAD Pseudoemiliania lacunosa (0.46 Ma) and extrapolations prior to the biostratigraphic boundary (Fig. 3).
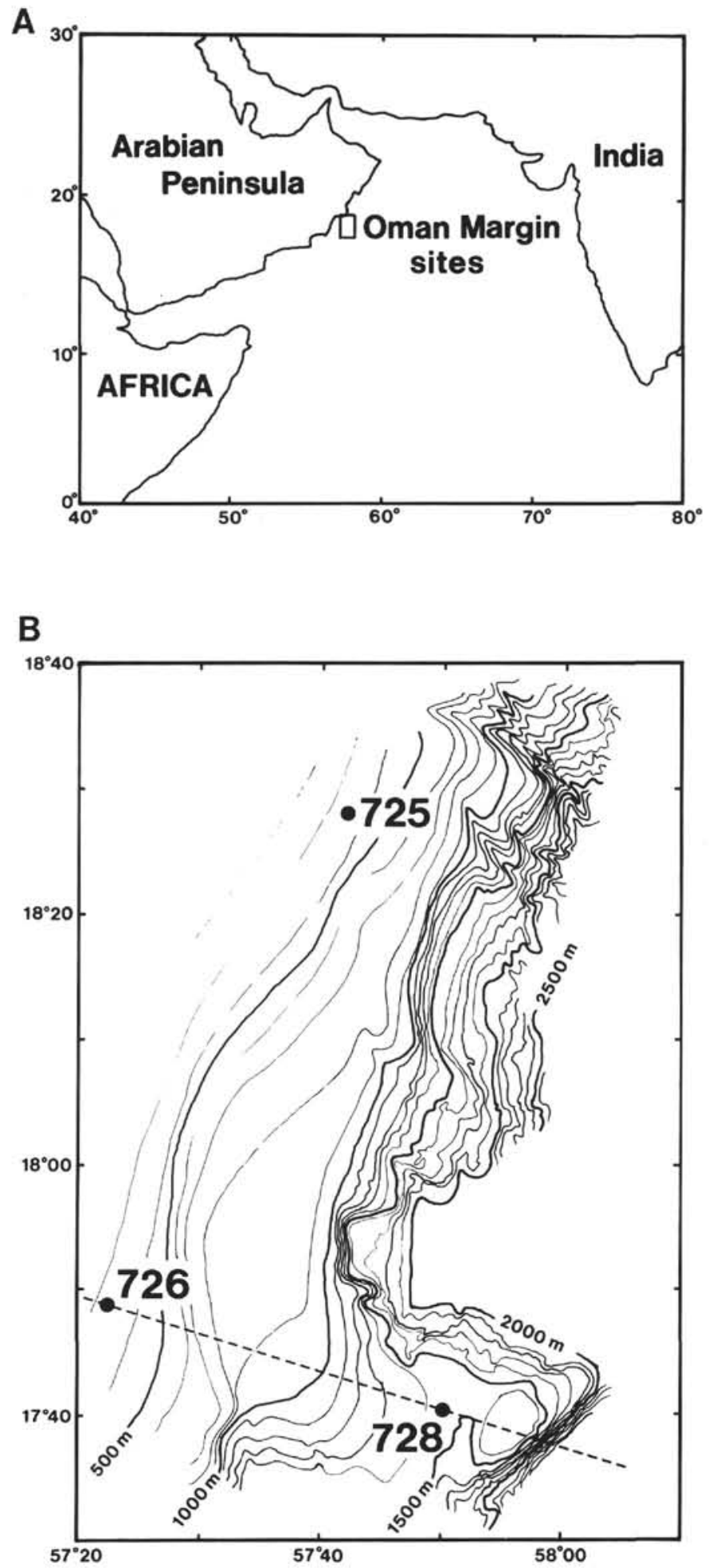

Figure 1. Location of Ocean Drilling Program Leg 117 Oman margin sites. A. Location map of the Oman margin in the northwestern Indian Ocean. B. Location of Sites $725, \mathbf{7 2 6}$, and 728 on the Oman margin. The broken line shows the approximate location of the idealized depth transect depicted in Figure 2. Bathymetry is from Mountain and Prell (1989). 


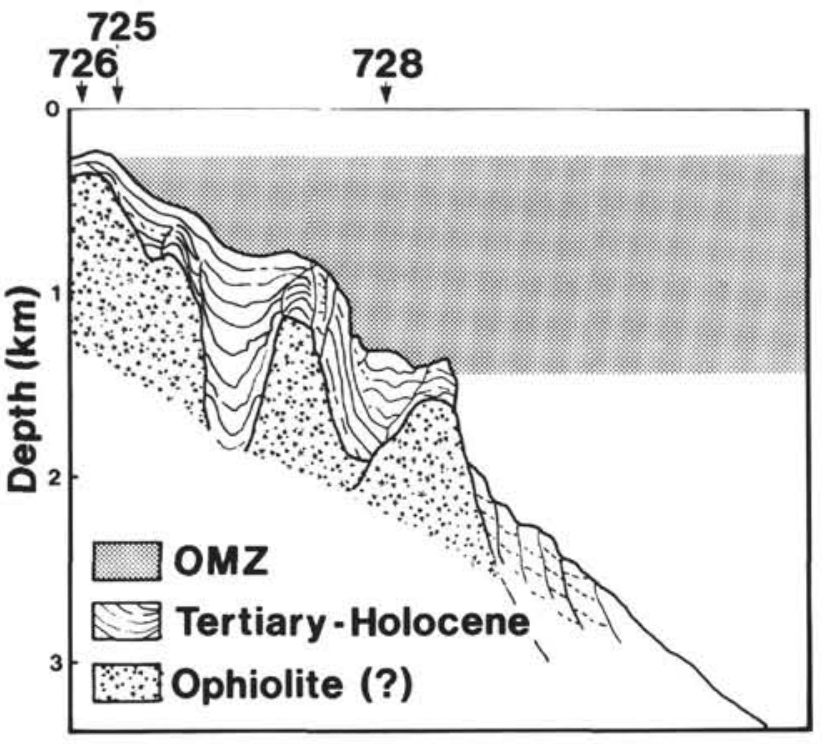

Figure 2. Schematic profile showing the structure of the Oman margin and the oxygen-minimum zone (OMZ).

The sedimentation rates at Site 726 were calculated as interpolations between the sediment-water interface, three calcareous nannofossil datum levels, one magnetostratigraphic boundary, and one planktonic foraminiferal datum level. The sedimentation rate is calculated to be $1.5 \mathrm{~cm} / \mathrm{k}$.y. prior to $3.5 \mathrm{Ma}$. In the interval $3.5-1.45 \mathrm{Ma}$ the sedimentation rate decreases to $0.6 \mathrm{~cm} / \mathrm{k}$.y. whereafter it increases and varies between 5.7 and $2.5 \mathrm{~cm} / \mathrm{k}$.y. in the interval 1.45-0.0 Ma (Fig. 4).

The rates of sediment accumulation in Site 728 were calculated as interpolations between the sediment-water interface, two magnetostratigraphic boundaries, two calcareous nannofossil datum levels, and two radiolarian datum levels. The sedimentation rate varies highly in the studied sequence with a sedimentation rate calculated to be $2.6 \mathrm{~cm} / \mathrm{k}$.y. prior to $5.70 \mathrm{Ma}$. It increases to $8.7 \mathrm{~cm} / \mathrm{k} . \mathrm{y}$. in the interval $5.70-3.56 \mathrm{Ma}$, whereafter it decreases to $1.4 \mathrm{~cm} / \mathrm{k}$.y. in the interval $1.89-2.92 \mathrm{Ma}$. After $2.92 \mathrm{Ma}$ the sedimentation rate increases again and reaches $4.2 \mathrm{~cm} / \mathrm{k} . \mathrm{y}$. in the interval 0.46-Recent (Fig. 5).

\section{RESULTS}

\section{ODP Site 725}

The analyzed section of Hole $725 \mathrm{C}$ represents Pleistocene through Recent. The number of specimens per cubic centimeter is variable at this site, with a high of nearly 28,000 and a low less than 10 (Fig. 6).

Analysis of the benthic foraminifers reveals three different faunal assemblages (Fig. 7). The oldest assemblage is characterized by Bolivina ordinaria, Bolivina seminuda, Bolivina sp. 2, Bulimina denudata, Bulimina subornata, Buliminella elegantissima, Globobulimina sp. 1, Hyalinea balthica, Lenticulina iota, and Uvigerina peregrina. A change in the faunal composition occurs at about $\approx 105 \mathrm{mbsf}(0.90 \mathrm{Ma})$. A faunal assemblage characterized by Bolivina ordinaria, Bolivina seminuda, Bulimina marginata, Buliminella elegantissima, Florilus sp. 2, Hyalinea balthica, Trifarina angulosa, and Uvigerina peregrina dominates the fauna until the youngest faunal assemblage appears at about $\approx 50 \mathrm{mbsf}(0.45 \mathrm{Ma})$ and continues to the $\mathrm{Re}$ cent. This assemblage is characterized Bolivina ordinaria, Bolivina spathulata, Bulimina sp. 2, Cassidulina laevigata, Elphi- dium advenum, Florilus sp. 3, Globobulimina sp. 1, Hanzawaia concentrica, Hoeglundina elegans, Spiroloculina sp. 1, Trifarina angulosa, Uvigerina peregrina, and Virgulina sp. 1. The transition to the youngest faunal assemblage is characterized by low frequencies of all species, except for Bolivina seminuda, which shows a strong dominance with a relative frequency of $85 \%$.

\section{ODP Site 726}

The analyzed section of Hole $726 \mathrm{~A}$ represents Eocene through Recent, with a major hiatus between the Eocene and upper Miocene. The number of specimens per cubic centimeter is difficult to calculate for the Eocene sequence due to strong lithification. It is very high in the Miocene interval with frequencies of up to 50,000 specimens $/ \mathrm{cm}^{3}$ (Fig. 8). At the Miocene/Pliocene boundary the relative number of specimens decreases to about 10,000 specimens $/ \mathrm{cm}^{3}$. At about $3 \mathrm{Ma}$ another decrease in the number of specimens occurs, down to an average of about 1,500 specimens $/ \mathrm{cm}^{3}$ (it varies between 300 and 6,600) (Fig. 8). The benthic foraminifers at Site 726 do not show any significant clustering into faunal assemblages through time, although significant variation in the relative abundance pattern of several species occurs.

The bottom part of the cored section (below $131.1 \mathrm{mbsf}$ ) is composed of partly dolomitized shallow-water limestone, greater than $96 \%$ carbonate. It contains abundant Eocene foraminifers with numerous nummulitiids. Among species recognized are Nummulites $\mathrm{cf}$. planulatus and Nummulites $\mathrm{cf}$. pratti. The presence of specimens of the genus Actinocyclina in a thin section from Sample 117-726A-16X-CC (148.1 mbsf) confirms this age.

Above the hiatus at $131.1 \mathrm{mbsf}$, the sediment is upper Miocene to Recent in age. In the interval 131.1-32.1 mbsf (approximately $7.5-0.7 \mathrm{Ma}$ ), it consists of a suite of organic-rich silty clays to marly nannofossil ooze to chalk with intercalated foraminifer-rich muds. The foraminifer-rich muds represent lag deposits and these form a composite lag bed in the lower part of this interval which is late Miocene in age. The lag deposits consist of phosphorite concretions, fish bones and fish teeth, and foraminifer tests with phosphatic incrustations. Genera present are Bolivina, Bulimina, and Uvigerina.

In the interval 121.4-111.8 mbsf (6.6-6.0 Ma), the benthic foraminiferal fauna is strongly dominated by Bolivina seminuda (relative abundance of about 95\%) (Fig. 9). The foraminiferal tests show signs of severe etching and occasional overgrowth.

In the interval 102.1-73.2 mbsf (5.4-3.4 Ma), the benthic foraminiferal fauna is strongly dominated by Bolivina ordinaria. This species is also present above this interval, although in much lower frequency (Fig. 9). Sample 9X-2, 57-59 cm (75.3 mbsf; 3.6 Ma) consists of a typical lag deposit with heavily abraded foraminifers, fish bones, and fish teeth with phosphatic incrustations together with phosphoritic concretions.

The interval above $68.7 \mathrm{mbsf}$ (2.6 Ma-Recent) is characterized by relatively high percentages of the species Uvigerina peregrina. Other species are also present, if not in the entire interval, they exhibit single peaks in their relative abundance patterns at various time intervals. Species showing high relative abundances within this interval are Bolivina seminuda, Bolivina spathulata, Bulimina denudata, Buliminella elegantissima, Globobulimina spp., Lenticulina iota, and Trifarina angulosa (Fig. 9). This interval has a higher clay content and a higher content of organic carbon than the other intervals.

\section{ODP Site 728}

The analyzed section of Hole $728 \mathrm{~A}$ represent the late Miocene through Pleistocene. The number of specimens per cubic centimeter is low in the Miocene/early Pliocene interval (about 100 ), increases slightly at $4.0 \mathrm{Ma}$, and reaches about 400 at approximately $2.5 \mathrm{Ma}$ (Fig. 10). 
Table 1. Census data for the benthic foraminiferal species from ODP Hole $725 \mathrm{C}$ in the $>125 \mu \mathrm{m}$ fraction. Frequencies are expressed as percent of total benthic foraminiferal fauna in increments of $5 \%$, i.e., $10 \%$ covers the frequency interval $7.5 \%-12.5 \%$.

\begin{tabular}{|c|c|c|c|c|c|c|c|c|c|c|c|c|c|c|c|c|c|c|c|c|c|c|c|c|c|c|c|}
\hline $\begin{array}{l}\text { Core, section, } \\
\text { interval }(\mathrm{cm})\end{array}$ & $\begin{array}{l}\text { Depth } \\
\text { (mbsf) }\end{array}$ & $\begin{array}{l}\text { Age } \\
\text { (Ma) }\end{array}$ & 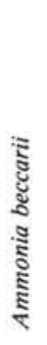 & 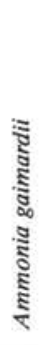 & 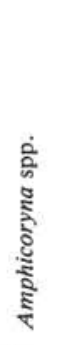 & 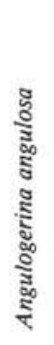 & 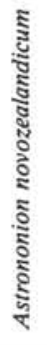 & 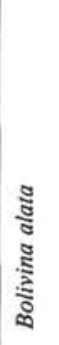 & 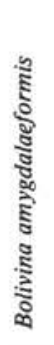 & 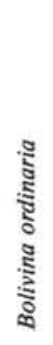 & 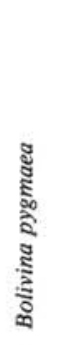 & 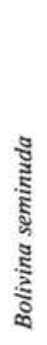 & 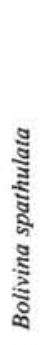 & 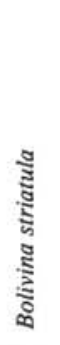 & 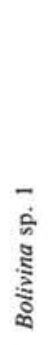 & 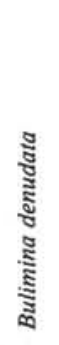 & 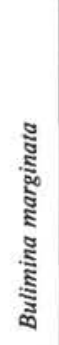 & 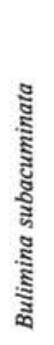 & 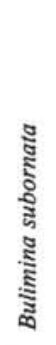 & 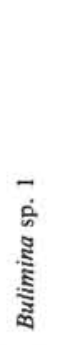 & 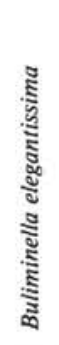 & 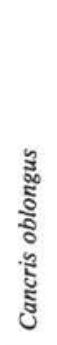 & $\begin{array}{l}\bar{b} \\
\dot{5} \\
\text { yे } \\
\text { š }\end{array}$ & 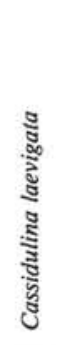 & 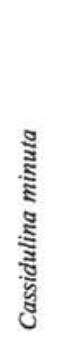 & 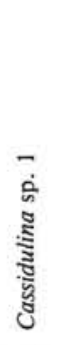 & 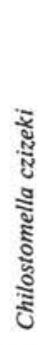 \\
\hline $1 \mathrm{H}-2,56-58$ & 2.07 & 0.02 & 1 & & 1 & 5 & & & 1 & 1 & & 1 & 1 & 1 & 1 & 1 & & 10 & & & 1 & & 1 & & & 1 & \\
\hline $1 \mathrm{H}-4,56-58$ & 5.07 & 0.05 & i & 1 & 1 & & & & 1 & 5 & & i & 5 & & & & & 10 & & 1 & & 1 & & 1 & & $i$ & \\
\hline $1 \mathrm{H}, \mathrm{CC}$ & 9.00 & 0.08 & & & 1 & & & & & 35 & & 1 & 5 & & & & & 10 & & 1 & & 1 & 1 & 5 & 1 & & 1 \\
\hline $2 \mathrm{H}-2,55-57$ & 11.06 & 0.10 & 1 & 5 & 1 & & 1 & & 5 & 1 & & & 5 & & & & & 1 & & & & 1 & 5 & 1 & 1 & 1 & 1 \\
\hline $2 \mathrm{H}-4,55-57$ & 14.06 & 0.13 & & & 1 & & & & & 5 & & 5 & 5 & & & & & & & 1 & & & & 1 & & 1 & \\
\hline $2 \mathrm{H}, \mathrm{CC}$ & 18.60 & 0.17 & & 1 & i & & & & & 1 & & & 5 & & & & & 5 & & & & 1 & 1 & 25 & & & \\
\hline $3 \mathrm{H}-2,55-57$ & 20.66 & 0.19 & & & & & & & & & 10 & & 1 & & 1 & & & 5 & & 15 & & & & 1 & & 1 & \\
\hline $3 \mathrm{H}-4,55-57$ & 23.66 & 0.21 & & & 1 & & & & & 20 & 1 & & 5 & & & 1 & & 20 & & 1 & & & & 5 & & & 1 \\
\hline $3 \mathrm{H}, \mathrm{CC}$ & 28.50 & 0.26 & 1 & & & & & & 1 & 10 & & & 5 & & & & & 5 & & 1 & & 1 & & 1 & & 1 & \\
\hline $4 \mathrm{H}-2,55-57$ & 30.56 & 0.28 & & & & 1 & 1 & & & 10 & 1 & 5 & 1 & & & & & 1 & & 1 & & & 1 & 1 & & 1 & \\
\hline $4 \mathrm{H}, \mathrm{CC}$ & 38.00 & 0.34 & & & 1 & 5 & 1 & & 1 & 10 & & 25 & 1 & & & & & 1 & & & 1 & & & i & & & \\
\hline $5 \mathrm{H}, \mathrm{CC}$ & 47.50 & 0.43 & & & & & & & 1 & & & 85 & 1 & & & & & 1 & & & & & & & & & \\
\hline $6 \mathrm{H}-2,55-57$ & 49.56 & 0.45 & & 1 & & & & & 1 & & & 15 & 5 & & & 1 & & & & 1 & 1 & 1 & & 1 & & & \\
\hline $6 \mathrm{H}-4,55-57$ & 52.56 & 0.47 & & & & & & & & & & 75 & 5 & & & 1 & 1 & & & & 1 & 1 & & 1 & & & \\
\hline $6 \mathrm{H}, \mathrm{CC}$ & 57.00 & 0.51 & & & & & & & & 1 & 1 & 85 & 1 & & & & i & & & & 5 & i & & 1 & & & \\
\hline $7 \mathrm{X}, \mathrm{CC}$ & 66.50 & 0.60 & & & & & & & & 60 & & 10 & 1 & & & & & & & & 10 & & & 1 & & & \\
\hline $8 X-2,55-57$ & 68.56 & 0.62 & & 1 & 1 & & & & & 55 & & 1 & 1 & & & & 5 & & & & 1 & 1 & & $i$ & & 1 & \\
\hline $8 \mathrm{X}, \mathrm{CC}$ & 76.10 & 0.69 & & & & & & & & 55 & & & $i$ & & & 1 & 1 & & & 1 & i & & & $i$ & & & \\
\hline $9 \times-2,55-57$ & 78.16 & 0.71 & & & 1 & & & 1 & & 25 & & 45 & & & & 1 & 1 & & & 1 & 10 & 1 & & 1 & & & \\
\hline $9 \times-4,55-57$ & 81.16 & 0.73 & & & 1 & & & & & 1 & & 85 & & & 1 & & & & & i & 5 & & & $i$ & & & \\
\hline $9 \mathrm{X}, \mathrm{CC}$ & 85.70 & 0.77 & & & 5 & & 1 & & 1 & 5 & & 1 & & & & 5 & 20 & & & & 1 & & & & & & \\
\hline $10 \mathrm{X}, \mathrm{CC}$ & 95.30 & 0.86 & & & 1 & & & & & 5 & 1 & 30 & 1 & & & 5 & 1 & & & 1 & 5 & 1 & & & & 1 & \\
\hline $11 \times-2,55-57$ & 97.36 & 0.88 & & & & & & & & 5 & & 55 & 1 & & & 10 & 15 & & & & 5 & & & 1 & & & \\
\hline $11 \times-4,55-57$ & 100.36 & 0.91 & & & & & & & & 15 & & 50 & 1 & & 1 & & 1 & 1 & & & 5 & 1 & & 1 & & & \\
\hline $11 \mathrm{X}, \mathrm{CC}$ & 104.90 & 0.95 & & & & & & & & 35 & & 25 & & & 1 & 1 & 1 & & 1 & & 10 & 1 & & 5 & & 1 & \\
\hline $12 X-2,55-57$ & 106.96 & 0.97 & & & & & & & & 5 & & 5 & & & 10 & 1 & & 1 & 10 & & 1 & $i$ & & & & 5 & 1 \\
\hline $12 \times-4,55-57$ & 109.96 & 0.99 & & & & & & & & 10 & & 20 & & & 1 & 5 & & & & 1 & 5 & 1 & & 5 & & 1 & \\
\hline $12 \mathrm{X}, \mathrm{CC}$ & 114.50 & 1.03 & & & & & & & & 1 & & 36 & & & & 15 & & & 5 & 5 & 5 & & & & & & \\
\hline $13 \times-2,55-57$ & 118.56 & 1.05 & & & & & & & & 5 & & 60 & & & 1 & 1 & & & 1 & & 5 & & & 1 & & 1 & \\
\hline $13 \mathrm{X}, \mathrm{CC}$ & 124.10 & 1.12 & & & & & & & & 1 & & 15 & & & 5 & 5 & & 1 & i & & 50 & & & & & 5 & \\
\hline $14 \times-2,55-57$ & 126.16 & 1.14 & & 1 & & & & & & 1 & 1 & 90 & & & 1 & 1 & & & & & 1 & & & 1 & & & \\
\hline $14 \times-4,55-57$ & 129.16 & 1.17 & & & & & & & & 1 & & 60 & & & 1 & & & & 1 & & 10 & & & & & & \\
\hline $14 \mathrm{X}, \mathrm{CC}$ & 133.80 & 1.21 & & & & & & & & 15 & 1 & 15 & & & 5 & 5 & & & 1 & 1 & 5 & & & 1 & & & \\
\hline $15 \mathrm{X}-2,55-57$ & 135.86 & 1.23 & & 1 & & & & & & 5 & & 55 & & & & 5 & & & & 5 & 10 & & & & & & 1 \\
\hline $15 \times-4,55-57$ & 138.86 & 1.25 & & & & & & & & 1 & & 20 & & & & & & & & & & & & 1 & & & \\
\hline $15 \mathrm{X}, \mathrm{CC}$ & 143.50 & 1.30 & & & & & & & & 70 & 1 & 1 & & & 5 & 5 & & & 1 & & & & & & & & \\
\hline $16 \mathrm{X}-2,55-57$ & 145.56 & 1.31 & & & 1 & & & & & 30 & & 1 & 1 & & & 1 & 1 & & & & 1 & 1 & & 5 & & & \\
\hline $16 \mathrm{X}, \mathrm{CC}$ & 153.20 & 1.38 & & & & & & & & 20 & 1 & 1 & & & 1 & 5 & & & 1 & & 1 & & & & & & \\
\hline $17 \mathrm{X}-2,55-57$ & 155.26 & 1.40 & & & & & & & & 35 & $i$ & & & & & 1 & & & & & 35 & & & 1 & & & \\
\hline $17 X-4,38-40$ & 158.09 & 1.43 & & & & & & & & 45 & & & & & 1 & & 1 & & & & 1 & & & 5 & & & \\
\hline $17 \mathrm{X}, \mathrm{CC}$ & 162.80 & 1.47 & & & & & & & & 80 & 1 & & & & 1 & & & & & & 1 & & & & & & \\
\hline
\end{tabular}


Table 1 (continued).

\begin{tabular}{|c|c|c|c|c|c|c|c|c|c|c|c|c|c|c|c|c|c|c|c|c|c|c|c|c|c|c|c|}
\hline $\begin{array}{l}\text { Core, section, } \\
\text { interval }(\mathrm{cm})\end{array}$ & $\begin{array}{l}\text { Depth } \\
\text { (mbsf) }\end{array}$ & $\begin{array}{l}\text { Age } \\
\text { (Ma) }\end{array}$ & 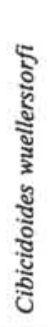 & 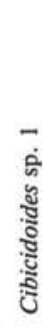 & 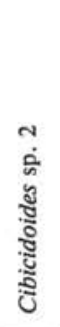 & 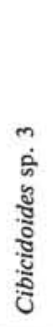 & 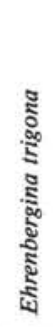 & 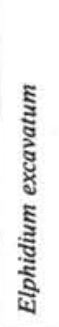 & 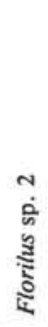 & $\begin{array}{l}m \\
\dot{0} \\
\text { s. } \\
\text { ș } \\
\text { s. }\end{array}$ & 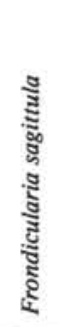 & 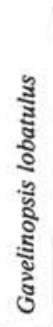 & 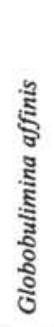 & 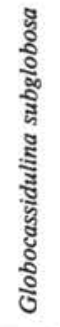 & 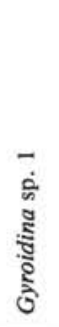 & 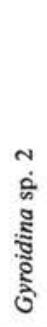 & 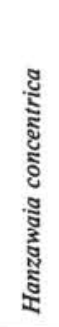 & 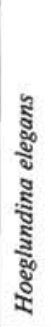 & 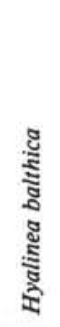 & 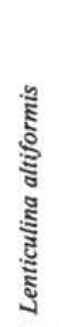 & 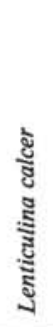 & 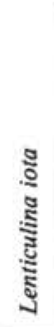 & 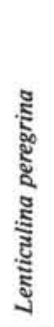 & 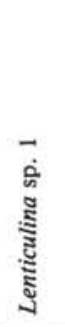 & 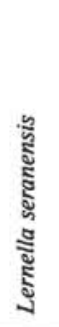 & 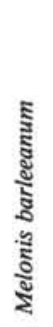 & 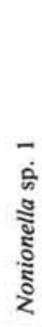 \\
\hline $1 \mathrm{H}-2,56-58$ & 2.07 & 0.02 & & 1 & 1 & & & 5 & & 5 & 5 & & & 1 & & 1 & 10 & 5 & 1 & 5 & 1 & & 1 & & & & \\
\hline $1 \mathrm{H}-4,56-58$ & 5.07 & 0.05 & & 5 & & & & 5 & 1 & 5 & 5 & 1 & & 5 & & 5 & 10 & 5 & 1 & 1 & & & & & & & \\
\hline $1 \mathrm{H}, \mathrm{CC}$ & 9.00 & 0.08 & & & & & & & 1 & 1 & 5 & 1 & 1 & 1 & & 1 & 15 & 5 & & 1 & 1 & & 1 & & & & \\
\hline $2 \mathrm{H}-2,55-57$ & 11.06 & 0.10 & & 1 & & & & 1 & & & 10 & & 1 & 5 & & & 15 & 5 & & 5 & & & & & & & \\
\hline $2 \mathrm{H}-4,55-57$ & 14.06 & 0.13 & & & & & & & & & & & 5 & & & & & & & 5 & & 1 & & & & & \\
\hline $2 \mathrm{H}, \mathrm{CC}$ & 18.60 & 0.17 & & 1 & 1 & & 20 & 5 & 1 & & 1 & 1 & & & 1 & & 10 & 1 & & 1 & 1 & & & & & & \\
\hline $3 \mathrm{H}-2,55-57$ & 20.66 & 0.19 & & & & & & 1 & 1 & & 1 & & 5 & 1 & & & 1 & & & 1 & 1 & & & & & & \\
\hline $3 \mathrm{H}-4,55-57$ & 23.66 & 0.21 & & & & & & i & 1 & 5 & 1 & & 1 & & & 1 & 5 & 1 & & 1 & 1 & & & & & & \\
\hline $3 \mathrm{H}, \mathrm{CC}$ & 28.50 & 0.26 & & 1 & & & & $i$ & 1 & & 5 & & & 1 & & 5 & 10 & i & & 1 & i & & & & 1 & & \\
\hline $4 \mathrm{H}-2,55-57$ & 30.56 & 0.28 & & & & & & 1 & & 1 & & & 5 & 1 & & & 5 & i & & 1 & 1 & & 1 & & & & \\
\hline $4 \mathrm{H}, \mathrm{CC}$ & 38.00 & 0.34 & & 1 & 1 & 1 & & 1 & & 1 & 1 & & & 1 & & 1 & 5 & 1 & 5 & 1 & 1 & & & & & & \\
\hline $5 \mathrm{H}, \mathrm{CC}$ & 47.50 & 0.43 & 1 & 1 & i & & & & & 1 & 1 & & & & & & 1 & & 1 & $i$ & & & & & & 1 & \\
\hline $6 \mathrm{H}-2,55-57$ & 49.56 & 0.45 & & $i$ & i & & & 1 & 1 & & i & & & 1 & & & 5 & 1 & 15 & 5 & & & 1 & & 1 & i & \\
\hline $6 \mathrm{H}-4,55-57$ & 52.56 & 0.47 & & & & 1 & & & & & & & & & & & 5 & & 1 & & & & & & & & \\
\hline $6 \mathrm{H}, \mathrm{CC}$ & 57.00 & 0.51 & & 1 & & & & 1 & & & & & & & & & 1 & & & 1 & & 1 & 1 & & & & \\
\hline $7 \mathrm{X}, \mathrm{CC}$ & 66.50 & 0.60 & & & & & & & 1 & & & & & 1 & & & i & & & & & & & & & & \\
\hline $8 X-2,55-57$ & 68.56 & 0.62 & & & & & 1 & 1 & $i$ & & & & & 1 & & & 1 & & 5 & 1 & 1 & 5 & & & & & \\
\hline $8 \mathrm{X}, \mathrm{CC}$ & 76.10 & 0.69 & & 1 & & & & i & 5 & & & & & $i$ & & & 5 & & 15 & $i$ & & & & & & & \\
\hline $9 X-2,55-57$ & 78.16 & 0.71 & & & & & & & 5 & & & & & 1 & & & 1 & & 5 & 1 & & & 1 & & & & \\
\hline $9 \times-4,55-57$ & 81.16 & 0.73 & & & & & & & & & & & & $i$ & & & 1 & & & & 1 & & & & & & \\
\hline $9 \mathrm{X}, \mathrm{CC}$ & 85.70 & 0.77 & & & & & & & 1 & & & & & & & & 5 & & & 5 & i & & & & & 1 & 1 \\
\hline $10 \mathrm{X}, \mathrm{CC}$ & 95.30 & 0.86 & & & & 1 & & & 5 & & & & & & & & 5 & & 10 & 5 & i & & & & & 1 & \\
\hline $11 \times-2,55-57$ & 97.36 & 0.88 & & & & & & & 1 & & & & 1 & & & & 1 & & 1 & & & & & & & & 1 \\
\hline $11 \times-4,55-57$ & 100.36 & 0.91 & & & & & & & 5 & & & & & & & & 5 & & 1 & 1 & & & & & & & 1 \\
\hline $11 \mathrm{X}, \mathrm{CC}$ & 104.90 & 0.95 & 1 & & & & & & & & & & 1 & & & & 1 & & 5 & 1 & & & & & & & \\
\hline $12 X-2,55-57$ & 106.96 & 0.97 & & & & & & & & & & & 5 & & & & & & 20 & 5 & & & & & & & 1 \\
\hline $12 \times-4,55-57$ & 109.96 & 0.99 & & & & & & & 1 & & & & 1 & & & & 5 & & 5 & 5 & & 1 & & & & & \\
\hline $12 \mathrm{X}, \mathrm{CC}$ & 114.50 & 1.03 & & 1 & & & & & & & & & i & & & & 1 & & 1 & 5 & & 5 & & & & & 1 \\
\hline $13 \mathrm{X}-2,55-57$ & 118.56 & 1.05 & & & & & & & & & & & 15 & & & & 1 & & 1 & 1 & & 1 & & & & & \\
\hline $13 \mathrm{X}, \mathrm{CC}$ & 124.10 & 1.12 & & & & & 5 & & & & & & 1 & & & & 1 & & 5 & 5 & & 5 & & & & & 1 \\
\hline $14 X-2,55-57$ & 126.16 & 1.14 & & & & & 1 & & 1 & & & & i & & & & i & & 1 & & & & & & & & \\
\hline $14 \times-4,55-57$ & 129.16 & 1.17 & & & & & 1 & & 1 & & & & 10 & & & & 1 & & 1 & 1 & & 5 & 1 & & & & 1 \\
\hline $14 \mathrm{X}, \mathrm{CC}$ & 133.80 & 1.21 & & & & & & 1 & & & & & 1 & & & & 1 & & 10 & 1 & & 5 & & 5 & & & 1 \\
\hline $15 X-2,55-57$ & 135.86 & 1.23 & & & & & & & & & & & & & & & & & 1 & & & 15 & & & & & \\
\hline $15 \times-4,55-57$ & 138.86 & 1.25 & & & & & & & & & & & 5 & & & & & & & & & 65 & & & & & \\
\hline $15 \mathrm{X}, \mathrm{CC}$ & 143.50 & 1.30 & & & & & & & & & & & & & & & 1 & & 10 & & & 1 & & & & & 1 \\
\hline $16 X-2,55-57$ & 145.56 & 1.31 & & & & & & & 1 & & & & & & & & 10 & & 15 & 1 & & & 1 & & & & \\
\hline $16 \mathrm{X}, \mathrm{CC}$ & 153.20 & 1.38 & & & & & & & & & & & 1 & & & & & & 1 & & & 60 & & & & & \\
\hline $17 \mathrm{X}-2,55-57$ & 155.26 & 1.40 & & & & & & & 1 & & & & & & & & 5 & & & 1 & & 15 & & & & & 1 \\
\hline $17 X-4,38-40$ & 158.09 & 1.43 & & & & & & & 1 & & & & 1 & & & & 1 & & 5 & & & 1 & & & & & \\
\hline $17 \mathrm{X}, \mathrm{CC}$ & 162.80 & 1.47 & & & & & & & & & & & & & & & 5 & & 5 & & & 1 & & & & & 1 \\
\hline
\end{tabular}


Table 1 (continued).

\begin{tabular}{|c|c|c|c|c|c|c|c|c|c|c|c|c|c|c|c|c|c|c|c|c|c|c|c|c|c|}
\hline $\begin{array}{l}\text { Core, section, } \\
\text { interval }(\mathrm{cm})\end{array}$ & $\begin{array}{l}\text { Depth } \\
\text { (mbsf) }\end{array}$ & $\begin{array}{l}\text { Age } \\
\text { (Ma) }\end{array}$ & 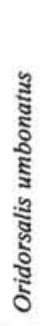 & 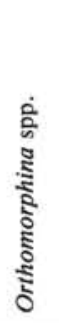 & 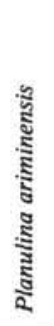 & 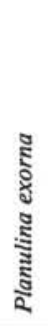 & 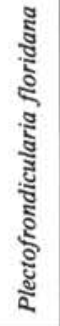 & 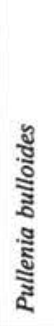 & 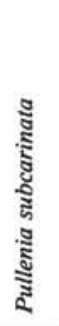 & 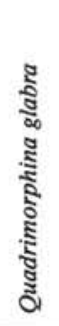 & 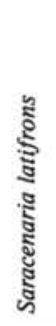 & 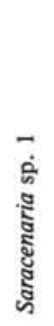 & 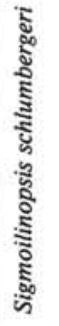 & 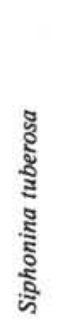 & 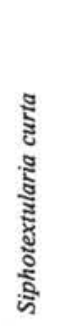 & 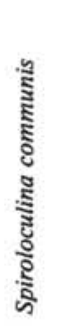 & 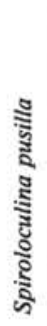 & 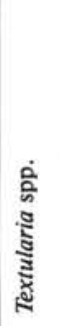 & 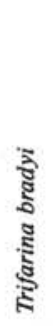 & 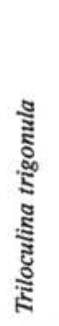 & 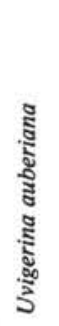 & 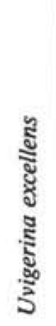 & 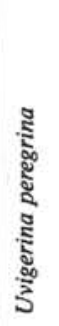 & 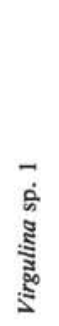 & 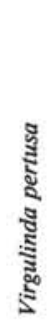 \\
\hline $1 \mathrm{H}-2,56-58$ & 2.07 & 0.02 & & & & 1 & & & & & & 1 & & & 5 & 5 & 1 & 1 & 1 & 1 & & 1 & 15 & & \\
\hline $1 \mathrm{H}-4,56-58$ & 5.07 & 0.05 & & & & & & & & & & & & & & 1 & 1 & & 1 & 1 & & & 15 & 1 & 10 \\
\hline $1 \mathrm{H}, \mathrm{CC}$ & 9.00 & 0.08 & & & & & & & & & & 1 & & & & & & & & 1 & & & 10 & & \\
\hline $2 \mathrm{H}-2,55-57$ & 11.06 & 0.10 & & & & 1 & & 1 & & & & & & & & & 1 & 1 & 10 & & & & 5 & 1 & \\
\hline $2 \mathrm{H}-4,55-57$ & 14.06 & 0.13 & & & & & & & & & & & & & & & 1 & & & 5 & & & 75 & & \\
\hline $2 \mathrm{H}, \mathrm{CC}$ & 18.60 & 0.17 & & 1 & & & & 1 & & 1 & & 1 & & & & 1 & & & 5 & & 1 & & & 1 & \\
\hline $3 \mathrm{H}-2,55-57$ & 20.66 & 0.19 & & & & & & & & & & 1 & & & 1 & & 1 & & & 1 & & & 1 & 5 & 60 \\
\hline $3 \mathrm{H}-4,55-57$ & 23.66 & 0.21 & & & & & & & & & & & & & & & 1 & 1 & 5 & 1 & & & 20 & & 1 \\
\hline $3 \mathrm{H}, \mathrm{CC}$ & 28.50 & 0.26 & & & & & & & & & & 1 & & & 1 & & & 1 & 20 & 1 & 1 & 1 & 15 & & 1 \\
\hline $4 \mathrm{H}-2,55-57$ & 30.56 & 0.28 & & & & & & & & & & & & & 1 & & 1 & & 5 & & & & 20 & 1 & 45 \\
\hline $4 \mathrm{H}, \mathrm{CC}$ & 38.00 & 0.34 & & & & & & & & & & 1 & & & & & & 1 & 20 & & 1 & 5 & 5 & & \\
\hline $5 \mathrm{H}, \mathrm{CC}$ & 47.50 & 0.43 & & & & & & 1 & & 1 & & & & & & & 1 & & 10 & & & & 1 & & \\
\hline $6 \mathrm{H}-2,55-57$ & 49.56 & 0.45 & & & & & & 1 & 1 & & & & 1 & & 5 & & 1 & & 25 & 1 & 1 & & 15 & & \\
\hline $6 \mathrm{H}-4,55-57$ & 52.56 & 0.47 & & & & & & 1 & & & 1 & 1 & & & & & & & 5 & & 1 & & 1 & & 5 \\
\hline $6 \mathrm{H}, \mathrm{CC}$ & 57.00 & 0.51 & & & & & & 1 & & & & 1 & & & & & & & & & & & 1 & & 1 \\
\hline $7 \mathrm{X}, \mathrm{CC}$ & 66.50 & 0.60 & & & & 1 & & & & & & & & & & & & & 5 & & & & 15 & & \\
\hline $8 \times-2,55-57$ & 68.56 & 0.62 & & & & 5 & & & & & & & 1 & & & & 1 & & & & 1 & & 15 & & \\
\hline $8 \mathrm{X}, \mathrm{CC}$ & 76.10 & 0.69 & & & & 1 & & 1 & & & & & & & & & 1 & & 5 & & & 1 & 5 & & \\
\hline $9 \times-2,55-57$ & 78.16 & 0.71 & & & & 1 & & & & & & & & & & & 1 & & & & & & 1 & & 1 \\
\hline $9 \times-4,55-57$ & 81.16 & 0.73 & & & & & & 1 & & & & & & & & & & & 1 & & & & 1 & & 1 \\
\hline $9 \mathrm{X}, \mathrm{CC}$ & 85.70 & 0.77 & & & 1 & & & 5 & & & & & & 1 & & & & & & 40 & & & 1 & 1 & \\
\hline $10 \mathrm{X}, \mathrm{CC}$ & 95.30 & 0.86 & & & & 1 & & 1 & & & 1 & & 1 & & & & 1 & & 5 & & & 1 & 10 & & 1 \\
\hline $11 \times-2,55-57$ & 97.36 & 0.88 & & & & & & & & & & & & & & & 1 & & & & & 1 & 5 & & 5 \\
\hline $11 X-4,55-57$ & 100.36 & 0.91 & & & & & & & & & & & & & & & & & 1 & & & 5 & 5 & & \\
\hline $11 \mathrm{X}, \mathrm{CC}$ & 104.90 & 0.95 & & & & 1 & & & & & & & & & & & & & & 1 & 1 & & 10 & & 1 \\
\hline $12 \times-2,55-57$ & 106.96 & 0.97 & & & & 1 & & 1 & & & 1 & 1 & & & & & 1 & & 1 & 1 & 1 & & 25 & & \\
\hline $12 \times-4,55-57$ & 109.96 & 0.99 & 1 & & & & & & & & & 1 & & & & & & & 10 & 1 & & 1 & 25 & & \\
\hline $12 \mathrm{X}, \mathrm{CC}$ & 114.50 & 1.03 & 1 & & & & & 1 & & & & 1 & & & & & & & 1 & & & & 20 & & 1 \\
\hline $13 \times-2,55-57$ & 116.56 & 1.05 & & & & 1 & & 1 & 1 & & & 1 & & & & & 1 & & 1 & & & & 5 & & 1 \\
\hline $13 \mathrm{X}, \mathrm{CC}$ & 124.10 & 1.12 & & & & 1 & & & & & & & & & & & 1 & & & 1 & & & 1 & & \\
\hline $14 X-2,55-57$ & 126.16 & 1.14 & & & & & & & & & & 1 & & & & & & & & & & & 5 & & \\
\hline $14 X-4,55-57$ & 129.16 & 1.17 & & & & 1 & & & & & & 1 & & & & & & & & & & & 10 & & \\
\hline $14 \mathrm{X}, \mathrm{CC}$ & 133.80 & 1.21 & & & & & 1 & 1 & & & & 1 & & & & & & & & 1 & & & 5 & & 1 \\
\hline $15 X-2,55-57$ & 135.86 & 1.23 & & & & & & & & & & $i$ & & & & & & & & & & & 1 & & \\
\hline $15 \times-4,55-57$ & 138.86 & 1.25 & & & & & & & & & & & & & & & & & & & & & 5 & & \\
\hline $15 \mathrm{X}, \mathrm{CC}$ & 143.50 & 1.30 & & & & & & & & & & & & & & & & & & & & & 1 & & \\
\hline $16 \times-2,55-57$ & 145.56 & 1.31 & & & & & & 5 & & & & & & & & & 1 & & 5 & 1 & 1 & & 5 & & \\
\hline $16 \mathrm{X}, \mathrm{CC}$ & 153.20 & 1.38 & 1 & & & & & & & & & & & & & & & & & & & & 5 & & \\
\hline $17 X-2,55-57$ & 155.26 & 1.40 & & & & & & & 1 & & & 1 & & & & & & & 1 & & & & 1 & & 5 \\
\hline $17 \times-4,38-40$ & 158.09 & 1.43 & & & & & & & & & & & & & & & & & 1 & & & & 40 & & \\
\hline $17 \mathrm{X}, \mathrm{CC}$ & 162.80 & 1.47 & & & & & & & & & & & & & & & & & & & & & 5 & & \\
\hline
\end{tabular}


The benthic foraminiferal fauna at Site 728 shows great variation through time, and distinct faunal assemblages can be recognized. The interval $341.9-\approx 128 \mathrm{mbsf}(7.9-3.9 \mathrm{Ma})$ is characterized by a late Miocene/early Pliocene assemblage dominated by Frondicularia sagittula, Hoeglundina elegans, Sphaeroidina bulloides, Sigmoilinopsis schlumbergeri, Uvigerina auberiana, and Uvigerina spinicostata. These species occur with high relative frequencies during this entire interval (Fig. 11). Within this interval a faunal association restricted to the Miocene can be recognized. This Miocene assemblage is found between 341.9 and $\approx 260$ mbsf (7.9-5.4 Ma) and includes the previously mentioned species, although it is dominated by Melonis barleeanum and Uvigerina peregrina (Fig. 12). In the lowermost samples of the interval the species Melonis pompilioides reaches very high relative abundances $(>40.0 \%)$ whereas the relative abundance of Melonis barleeanum decreases significantly in the same samples (Fig. 12). Other species reaching high relative frequencies during the late Miocene are Oridorsalis umbonatus and Sigmoilinopsis schlumbergeri. A distinctive early Pliocene assemblage dominated by Ammonia beccarii, Ehrenbergina trigona, and Pullenia bulloides occurs in the interval $\approx 260-\approx 128 \mathrm{mbsf}$ (5.4-3.9 Ma) (Fig. 13).

The interval $\approx 128-0$ mbsf (3.9 Ma-Recent) is characterized by a late Pliocene/Pleistocene assemblage dominated by Bolivina alata, Bolivina ordinaria, Bulimina aculeata, Bulimina truncana, Cassidulina leavigata, Gavelinopsis lobatulus, Globocassidulina subglobosa, and Hyalinea balthica (Fig. 14).

\section{Discussion}

Several species present in the two shallow sites (Sites 725 and 726) show similarities in their relative abundances during the Pleistocene. Both appearances and disappearances as well as peaks in the relative abundance pattern for several species are synchronous, which might indicate regional environmental influences rather than local subsidence or uplift events.

The changes seen in the benthic foraminiferal assemblages as well as in the lithology indicate that the environment at Site 726 has changed through time. The Miocene and Pliocene sediments at this site are characterized by frequent lag deposits interbedded with hemipelagic sediments. There is abundant phosphorite, believed to indicate current winnowing whereby the finegrained fraction of the sediment is eroded away, concentrating the phosphorite initially into coarse-grained sediments and finally into nodular deposits at $1.66 \mathrm{Ma}(63.60 \mathrm{mbsf})$. Above the lag deposit an almost 10-fold increase in the sediment accumulation rate from $0.6 \mathrm{~cm} / \mathrm{k} . y$. to $4.9 \mathrm{~cm} / \mathrm{k}$.y. occurs (see Fig. 4) as well as an increase in the planktonic/benthic ratio (Hermelin, unpubl. data). The cause for these changes might include oscillations of the top of the oxygen minimum zone, fluctuations in the intensity of the oxygen depletion, sea-level variations, and/ or the development of counter-currents in the upwelling system.

The strong dominance of Ammonia beccarii at Site 728 in the early Pliocene assemblage needs an explanation. The present depth of this site is $1436 \mathrm{mbsf}$, considerable deeper than most of the records of this species (Culver and Buzas, 1980; 1981; 1982). Normally this species inhabits the uppermost bathyal and neritic zones ( $<400 \mathrm{~m}$ of water). There are three possible causes for this early Pliocene occurrence of Ammonia beccarii: (1) redeposition of material as a result of gravitational processes, (2) changes in the environmental preference of Ammonia beccarii, and (3) subsidence of the site.

One explanation for the presence of Ammonia beccarii would be the reworking of material from topographically higher areas. Slumps from higher areas creating sediment thicknesses of 100 $150 \mathrm{~m}$ seem unlikely since sedimentological analysis indicates the sediment composition to be uniform, without turbidites, between 85 and 320 mbsf (Prell, Niitsuma, et al., 1989).

The second explanation would be a change in environmental preference of Ammonia beccarii. This seems unlikely since this species is known to be restricted to shallow-water environments and a change of the lower depth limit of over $1,000 \mathrm{~m}$ would certainly have been recognized elsewhere if true (Culver and $\mathrm{Bu}$ zas, 1980; 1981; 1982).

A third explanation would be tectonic movements that caused subsidence of Site 728 . Site 728 was located in the upper bathyal zone during the late Miocene. The water depth decreased in the early Pliocene and Site 728 was relocated to the neritic zone. In the middle and upper Pliocene an increase in water depth to the present upper middle bathyal zone occurred. The relative abundance pattern seen in the benthic foraminiferal species supports to some degree this explanation. The species of the late Pliocene/Pleistocene assemblage (Bolivina alata, Bulimina aculeata, Bulimina truncana, Cassidulina laevigata, Gavelinopsis lobatulus, and Hyalinea balthica), are all indicative of environments deeper than $1000 \mathrm{~m}$ (Hermelin and Shimmield, 1990), i.e., about the present water depth of Site 728 .

The species present prior to $3.5 \pm 0.2 \mathrm{Ma}$ (the Miocene, the early Pliocene, and the late Miocene/early Pliocene assemblages) mostly indicate shallower marine conditions. The species Ehrenbergina trigona and Hoeglundina elegans, belonging to the early Pliocene and late Miocene/early Pliocene assemblages, respectively, are abundant in the modern Arabian Sea between 300 and $1500 \mathrm{~m}$ (see data for Sites 725 and 726; Hermelin and Shimmield, 1990). The species Frondicularia sagittula (late Miocene/early Pliocene assemblage) is typical for the upper bathyal and neritic zones.

The presence of the suggested shallow-water early Pliocene assemblage relates well with a significant decrease in the planktonic/benthic ratio (Hermelin, unpubl. data) which together with the pattern seen in the benthic foraminiferal fauna supports the hypothesis of a significantly shallower location of Site 728 in late Miocene and early Pliocene. The increase in the water depth starts at this site at about $3.5 \mathrm{Ma}$.

The benthic foraminiferal faunas found at the three sites are all indicative of an area of upwelling. Changes in the faunal composition which occur through time may be attributed to changes in the upwelling/monsoonal intensity, but could as well be caused by Neogene tectonics of the Oman margin as seen at Site 728 .

\section{ACKNOWLEDGMENTS}

I thank all scientists from the shipboard party of ODP Leg 117 and the technical staff and the crew on Joides Resolution for their contribution to this successful cruise. I thank Dr. Altenbach and an anonymous reviewer for their comments and suggestions which improved the paper. Financial support from the Swedish Natural Science Research Council (NFR) is acknowledged.

\section{SYSTEMATIC PALEONTOLOGY}

The faunal reference list contains the preferred modern name of the species followed by its original name.

Ammonia beccarii $($ Linné) $=$ Nautilus beccarii Linné, 1758, Systema Naturae, p. 710.

Ammonia gaimardii (d'Orbigny) = Rotalia (Turbinulina) gaimardii d’Orbigny, 1826, Ann. Sci. Nat., ser. 1, vol. 7, p. 275, pl. 106, mod. 9. Ammomargulinulina foliacea (Brady) = Haplophragmium foliaceum Brady, 1881, Quart. J. Micr. Sci., vol. 20, p. 50.

Amphicoryna spp.

Angulogerina angulosa (Williamson) = Uvigerina angulosa Williamson, 1858, Ray Soc., London, p. 67, pl. 5, fig. 140. 
Table 2. Census data for the benthic foraminiferal species from ODP Hole 726A in the $>125 \mu \mathrm{m}$ fraction. Frequencies are expressed as percent of total benthic foraminiferal fauna in increments of $5 \%$, i.e., $10 \%$ covers the frequency interval $7.5 \%-12.5 \%$.

\begin{tabular}{|c|c|c|c|c|c|c|c|c|c|c|c|c|c|c|c|c|c|c|c|c|c|c|c|c|c|c|c|c|c|c|c|c|}
\hline $\begin{array}{l}\text { Core, section, } \\
\text { interval }(\mathrm{cm})\end{array}$ & $\begin{array}{l}\text { Depth } \\
\text { (mbsf) }\end{array}$ & $\begin{array}{l}\text { Age } \\
\text { (Ma) }\end{array}$ & 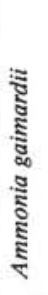 & 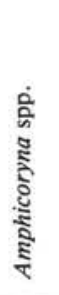 & 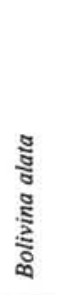 & 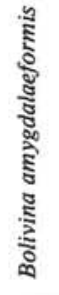 & 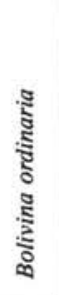 & 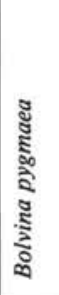 & 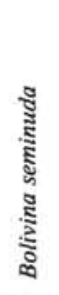 & 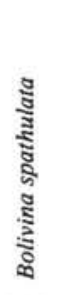 & 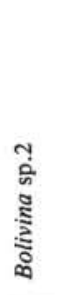 & 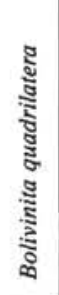 & 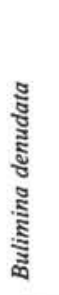 & 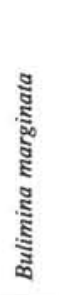 & 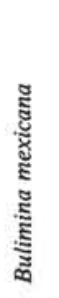 & 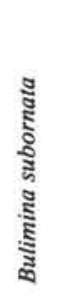 & 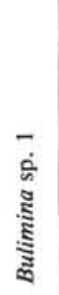 & $\begin{array}{l}m \\
\text { के } \\
\text { हू } \\
\text { है } \\
\text { ज्ञ }\end{array}$ & 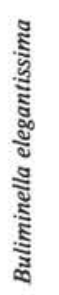 & 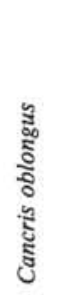 & 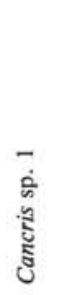 & 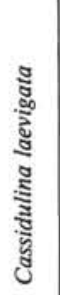 & 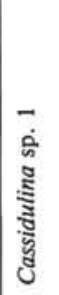 & 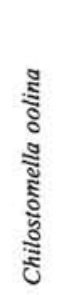 & 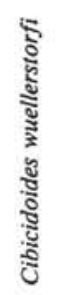 & 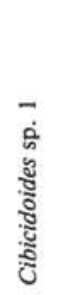 & 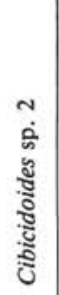 & 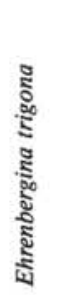 & 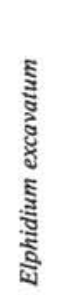 & 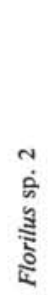 & 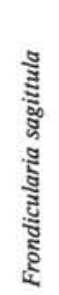 & 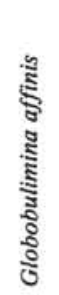 \\
\hline $1 \mathrm{H}-2,55-57$ & 2.06 & 0.04 & & & & 1 & 1 & & & 5 & 1 & & & 1 & & & & 15 & & & & & 1 & 1 & & 1 & 1 & & 5 & & 5 & \\
\hline $\mathrm{IH}, \mathrm{CC}$ & 6.90 & 0.12 & 1 & & & & 5 & & & 5 & & & & & & & & 15 & & 1 & 1 & 5 & $i$ & 1 & & $i$ & 5 & 1 & 5 & 1 & 5 & \\
\hline $2 \mathrm{H}-4,55-57$ & 11.96 & 0.21 & & & & 5 & 5 & & 5 & 10 & & & & & & & & 5 & & & 5 & & 1 & 1 & & & 1 & & & & & \\
\hline $3 \mathrm{H}-2,55-57$ & 18.36 & 0.32 & & & & 1 & 1 & 1 & 1 & 80 & & & & & & & & & 1 & 1 & 1 & 1 & 1 & 1 & & & & & & 1 & 1 & \\
\hline $3 \mathrm{H}, \mathrm{CC}$ & 25.80 & 0.45 & & & & & 20 & & 1 & 5 & & & 1 & & 1 & & & 1 & & 1 & & & 1 & 1 & & 1 & & 1 & & & & \\
\hline $4 \mathrm{H}-4,55-57$ & 30.86 & 0.65 & & & & 1 & 1 & & 5 & 1 & & & & & 25 & & & & 1 & 1 & & & & & & & & & & 1 & & \\
\hline $5 \mathrm{H}-2,55-57$ & 37.36 & 0.91 & & & & & 10 & & 15 & 1 & 1 & & 15 & & & & & 1 & i & $i$ & & 1 & 1 & & & & & & 1 & & & 1 \\
\hline $5 \mathrm{H}, \mathrm{CC}$ & 44.70 & 1.09 & & & & & & & 5 & & 5 & & 35 & & & 5 & & 1 & 5 & 1 & & 1 & & & & & & & & & & 1 \\
\hline $6 \mathrm{H}-4,55-57$ & 49.76 & 1.19 & & & & & & 1 & 50 & & 5 & & 5 & & & 1 & & & 15 & & & i & & & & & & 1 & & & & 20 \\
\hline $7 X-2,55-57$ & 56.16 & 1.32 & & 10 & & & & & 1 & & 1 & & 5 & & & & 15 & 1 & 5 & & & & & & & & & & & & & 10 \\
\hline $7 \mathrm{X}, \mathrm{CC}$ & 63.60 & 1.66 & & 1 & & & 25 & & & & & & 1 & & & 1 & & & 1 & & & 5 & & & & & & 5 & & 1 & & 1 \\
\hline $8 \times-2,55-57$ & 65.66 & 2.02 & & 1 & & & & & 15 & & 1 & & & & & & 20 & & 60 & & & & & & & & & & & & & 1 \\
\hline $8 X-4,55-57$ & 68.66 & 2.56 & & 1 & & & 5 & & 15 & & & & & & & & 40 & & 20 & & & & & & & & & & & & & \\
\hline $8 \mathrm{X}, \mathrm{CC}$ & 73.20 & 3.36 & & & & & 70 & & & & & & & & & & 1 & & & & & & & & & & & & & & & \\
\hline $9 \times-2,57-59$ & 75.28 & 3.57 & & & & & & & & & & & & & & & & & & & & & & & & & & & & & & \\
\hline $9 \mathrm{X}, \mathrm{CC}$ & 82.80 & 4.07 & & & 1 & & 40 & & 10 & & & & & & & & 10 & & 25 & & & & & & & & & & & & & \\
\hline $10 \times-2,55-57$ & 84.86 & 4.21 & & & & & 95 & & & & & & & & & & & & 1 & & & & & & & & & & & & & 1 \\
\hline $10 \mathrm{X}, \mathrm{CC}$ & 92.40 & 4.71 & & & 1 & & 35 & & & & 1 & & & & & & 1 & & 5 & & & 1 & 1 & & & & & & & & & \\
\hline $11 \times-4,55-57$ & 97.46 & 5.04 & & & i & & 60 & & 5 & & & & & & & & & & 10 & & & & & & & & & & & & & \\
\hline $11 \mathrm{X}, \mathrm{CC}$ & 102.10 & 5.35 & & & 5 & & 60 & & 5 & & & & & & & & & & 1 & & & 15 & 1 & & & & & & & & & \\
\hline $12 \mathrm{X}, \mathrm{CC}$ & 111.80 & 6.00 & & & & & & & 95 & & & & & & & & & & 5 & & & & & & & & & & & & & \\
\hline $13 \times-4,55-57$ & 116.86 & 6.33 & & & & & & & 95 & & & & & & & & & & 1 & & & & & & & & & & & & & \\
\hline $13 \mathrm{X}, \mathrm{CC}$ & 121.40 & 6.63 & & & & & & & 95 & & & & & & & & & & & & & & & & & & & & & & & \\
\hline
\end{tabular}


Table 2 (continued).

\begin{tabular}{|c|c|c|c|c|c|c|c|c|c|c|c|c|c|c|c|c|c|c|c|c|c|c|c|c|c|c|c|c|c|c|c|}
\hline $\begin{array}{l}\text { Core, section, } \\
\text { interval }(\mathrm{cm})\end{array}$ & $\begin{array}{l}\text { Depth } \\
\text { (mbsf) }\end{array}$ & $\begin{array}{l}\text { Age } \\
\text { (Ma) }\end{array}$ & 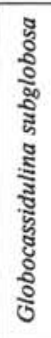 & 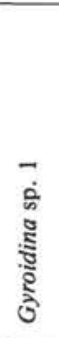 & 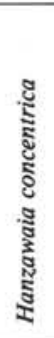 & 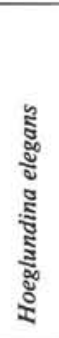 & 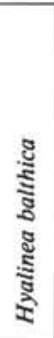 & 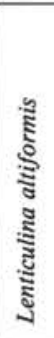 & 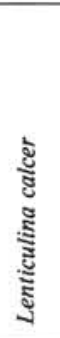 & 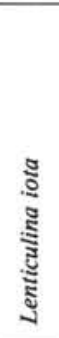 & 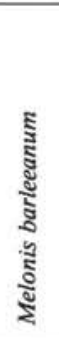 & 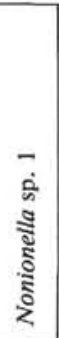 & 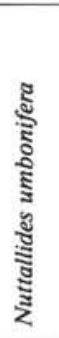 & 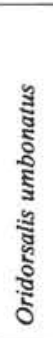 & 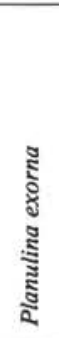 & 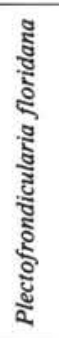 & 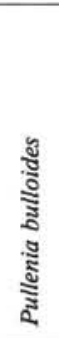 & 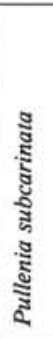 & 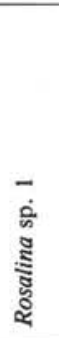 & 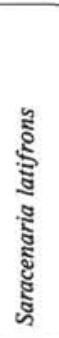 & 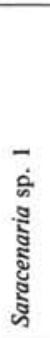 & 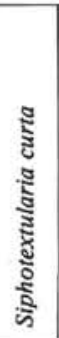 & 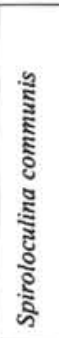 & 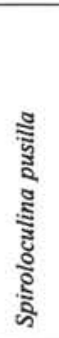 & 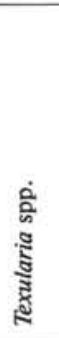 & 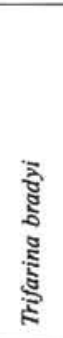 & 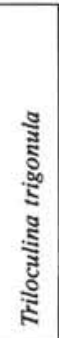 & 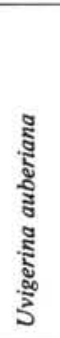 & 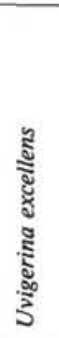 & 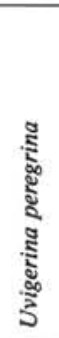 & 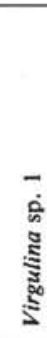 \\
\hline $1 \mathrm{H}-2,55-57$ & 2.06 & 0.04 & & & & & & & & & & & & & & & & & & & & & & & & & & & & & \\
\hline $\mathrm{HH}, \mathrm{CC}$ & 6.90 & 0.12 & 1 & & 15 & 5 & 5 & 1 & 1 & 1 & & & & & & & & & 5 & & & 5 & 5 & & & 1 & 5 & & 1 & 20 & \\
\hline $2 \mathrm{H}-4,55-57$ & 11.96 & 0.21 & & & 15 & 10 & & & 1 & & & & & & & & 1 & & 1 & & 1 & 5 & 1 & 1 & & 5 & 1 & 1 & & 10 & \\
\hline $3 \mathrm{H}-2,55-57$ & 18.36 & 0.32 & 10 & & 20 & 1 & & 1 & & & & & & & & & & & & & & & $i$ & i & & 20 & i & & & 5 & \\
\hline $3 \mathrm{H}, \mathrm{CC}$ & 25.80 & 0.45 & & & 5 & i & 1 & 1 & 1 & 1 & & & & & 1 & & 1 & & & & 1 & & i & & 5 & 1 & & & 5 & & \\
\hline $4 \mathrm{H}-4,55-57$ & 30.86 & 0.65 & & & 10 & & 5 & $i$ & 1 & i & & 1 & & & 5 & & i & 1 & & & & & & 1 & & 10 & & & 1 & 10 & \\
\hline $5 \mathrm{H}-2,55-57$ & 37.36 & 0.91 & & & 10 & & 1 & 5 & 1 & & 1 & & & & & & 5 & & 5 & & & & & 1 & & 30 & & 1 & & 1 & \\
\hline $5 \mathrm{H}, \mathrm{CC}$ & 44.70 & 1.09 & & & 5 & & $i$ & 5 & 1 & 5 & & 1 & & 1 & 1 & & 1 & & & & 1 & & & 1 & & 5 & 1 & & 1 & 20 & \\
\hline $6 \mathrm{H}-4,55-57$ & 49.76 & 1.19 & & & 1 & & 10 & 5 & & 5 & & & & i & i & & i & 1 & & & i & & & & & & i & & i & 15 & \\
\hline $7 \mathrm{X}-2,55-57$ & 56.16 & 1.32 & & & & & 1 & & & 5 & & & & & 1 & & & 1 & & & 1 & & & & & & & & & 1 & 1 \\
\hline $7 \mathrm{X}, \mathrm{CC}$ & 63.60 & 1.66 & & & 5 & & i & 1 & & 25 & & & & 5 & & & & 5 & & 1 & 5 & & & & & & 1 & & & 10 & \\
\hline $8 X-2,55-57$ & 65.66 & 2.02 & & & 10 & & 15 & 5 & & 10 & & & & & 1 & & & & & & & & & & & 5 & 1 & & & 20 & \\
\hline $8 X-4,55-57$ & 68.66 & 2.56 & & & & & 1 & & & & & & & & & & & & & & & & & & & & & & & 1 & 5 \\
\hline $8 \mathrm{X}, \mathrm{CC}$ & 73.20 & 3.36 & & & & & & & & 5 & & & & & & & & & & & & & & & & & & & & 10 & \\
\hline $9 X-2,57-59$ & 75.28 & 3.57 & & & & & & & & 5 & & 1 & & & & & & & & & & & & & & & & & & & 1 \\
\hline $9 \mathrm{X}, \mathrm{CC}$ & 82.80 & 4.07 & & & & & & & & & & & & & & & & & & & & & & & & & & & & & \\
\hline $10 X-2,55-57$ & 84.86 & 4.21 & & & & & & & & 15 & & & & & & & & & & & & & & & & & & & & 1 & \\
\hline $10 \mathrm{X}, \mathrm{CC}$ & 92.40 & 4.71 & & & 1 & & 1 & 1 & & 1 & & & & & 1 & & & & & & & & & & & & 1 & & 1 & & \\
\hline $11 X-4,55-57$ & 97.46 & 5.04 & 1 & & 10 & & 10 & & & 5 & & & 10 & & & & & & & & 1 & & & & & 1 & i & & 5 & 10 & \\
\hline $11 \mathrm{X}, \mathrm{CC}$ & 102.10 & 5.35 & & & 1 & & & 1 & & 1 & & & 1 & & & & & & & & & & & & & & & & 10 & 1 & 1 \\
\hline $12 \mathrm{X}, \mathrm{CC}$ & 111.80 & 6.00 & & & & & 5 & 5 & & i & & & & & & 1 & & & & & & & & & & & & & & 5 & 1 \\
\hline $13 \times-4,55-57$ & 116.86 & 6.33 & & & & & & & & & & & & & & & & & & & & & & & & & & & 1 & & 1 \\
\hline $13 \mathrm{X}, \mathrm{CC}$ & 121.40 & 6.63 & & 1 & & & & & & & & & & & & & & & & & & & & & & & & & & & 1 \\
\hline
\end{tabular}


Table 3. Census data for the benthic foraminiferal species from ODP Hole 726A in the $>125 \mu \mathrm{m}$ fraction. Frequencies are expressed as percent of total benthic foraminiferal fauna with the precision of one digit.

\begin{tabular}{|c|c|c|c|c|c|c|c|c|c|c|c|c|c|c|c|c|c|c|c|c|c|c|c|}
\hline $\begin{array}{l}\text { Core, section, } \\
\text { interval }(\mathrm{cm})\end{array}$ & $\begin{array}{l}\text { Depth } \\
\text { (mbsf) }\end{array}$ & $\begin{array}{l}\text { Age } \\
\text { (Ma) }\end{array}$ & 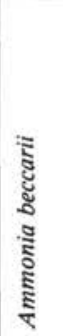 & 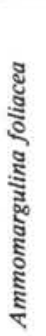 & 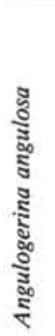 & 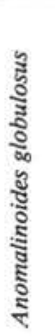 & 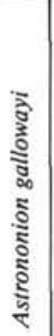 & 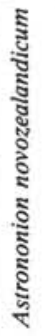 & 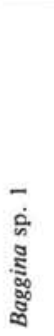 & 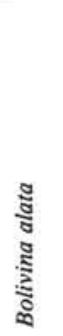 & 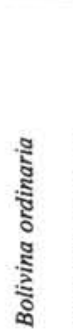 & 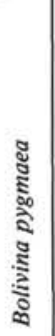 & 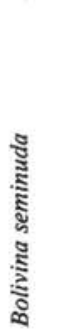 & 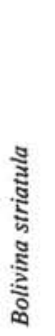 & 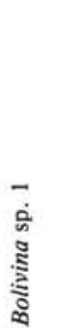 & 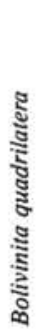 & 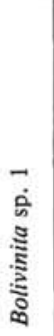 & 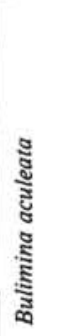 & 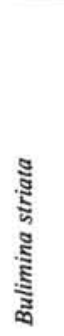 & 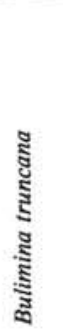 & 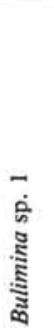 & 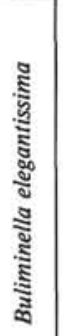 & 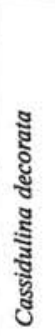 \\
\hline $1 \mathrm{H}-2,55-57$ & 2.06 & 0.05 & & & & & & & & 0.3 & & 0.3 & & & & & & 0.3 & 2.1 & 0.3 & 0.6 & 0.3 & \\
\hline $1 \mathrm{H}-4,55-57$ & 5.06 & 0.12 & & & & & & 3.8 & & 0.3 & 0.3 & & & & & & & 10.2 & 0.5 & 4.0 & & & \\
\hline $1 \mathrm{H}, \mathrm{CC}$ & 9.60 & 0.23 & & & 1.5 & & & 1.2 & & 1.2 & & & & & & & & 0.3 & 0.9 & 5.8 & 0.3 & & \\
\hline $2 \mathrm{H}-2,55-57$ & 11.66 & 0.28 & & & 0.8 & & & 0.5 & 0.3 & 0.8 & 0.3 & & & & & & & 1.0 & 0.8 & 2.6 & & 0.8 & \\
\hline $2 \mathrm{H}-4,55-57$ & 14.66 & 0.35 & 0.3 & & & & & 2.7 & & 1.1 & & 0.2 & & & & & & 11.4 & 1.1 & 1.4 & 0.6 & & 0.2 \\
\hline $2 \mathrm{H}, \mathrm{CC}$ & 19.10 & 0.45 & & & & & & & & 5.4 & & & & & & & & 5.4 & 0.6 & 3.2 & 0.3 & & \\
\hline $3 \mathrm{H}, \mathrm{CC}$ & 28.50 & 0.67 & & & 0.5 & & & & & 5.0 & 7.1 & & 0.3 & & & & & & 2.6 & 5.3 & & & \\
\hline $4 \mathrm{H}-4,55-57$ & 33.56 & 0.79 & & & & & & & & 12.4 & 12.2 & & & & & & 0.3 & 7.7 & 1.1 & 0.6 & & & \\
\hline $4 \mathrm{H}, \mathrm{CC}$ & 38.00 & 0.90 & & & & 0.5 & & 0.2 & & 3.2 & 1.5 & & & & & & & 15.9 & 1.5 & 2.0 & 0.5 & & \\
\hline $5 \mathrm{H}-2,55-57$ & 40.06 & 0.95 & & & & & & & & 10.8 & & & 0.4 & & & & 0.4 & 2.3 & 1.5 & 6.5 & 0.4 & & \\
\hline $5 \mathrm{H}-4,55-57$ & 43.06 & 1.04 & & & & & & & & 3.9 & 17.4 & & & & & & & 8.2 & 2.8 & 0.7 & 0.4 & & \\
\hline $5 \mathrm{H}, \mathrm{CC}$ & 47.50 & 1.21 & & & & 0.3 & & & & 3.5 & 5.7 & & 0.2 & & & & & 14.5 & 2.0 & 1.1 & 0.3 & & \\
\hline $6 \mathrm{H}-2,55-57$ & 49.56 & 1.29 & & & & & & 0.2 & & 0.4 & 1.2 & & & & & & 0.2 & 0.8 & 0.8 & 6.9 & 0.2 & & \\
\hline $6 \mathrm{H}, \mathrm{CC}$ & 57.00 & 1.58 & & & & & & & & 5.5 & 1.0 & & & & 3.1 & & & 4.7 & 0.8 & 3.4 & 0.3 & & 0.3 \\
\hline $7 \mathrm{H}-3,30-32$ & 60.31 & 1.71 & & & & 0.3 & & & & 1.0 & & & & & & & 0.3 & 11.6 & 2.1 & 4.9 & & & \\
\hline $7 \mathrm{H}-5,100-102$ & 64.01 & 1.85 & & & & & & & & 6.5 & 0.4 & & & & & & & 14.1 & 1.6 & 5.2 & & & 0.4 \\
\hline $7 \mathrm{H}, \mathrm{CC}$ & 66.50 & 1.98 & & & & & & & & 0.2 & & & & & 0.4 & 0.4 & & 0.9 & 0.7 & 8.3 & & 0.2 & 0.2 \\
\hline $8 \mathrm{H}-4,55-57$ & 71.56 & 2.35 & & & & & & & & 1.1 & & & & & & & 0.3 & 5.6 & 6.6 & 15.1 & & & \\
\hline $8 \mathrm{H}-6,55-57$ & 74.56 & 2.56 & & & & & & & & 0.9 & 0.6 & 0.3 & & & & & 0.9 & 0.9 & 12.1 & 7.4 & 0.3 & 0.9 & \\
\hline $8 \mathrm{H}, \mathrm{CC}$ & 76.10 & 2.67 & & & & & & & & 5.6 & 4.9 & & & & & 0.2 & & 0.2 & 9.0 & 3.7 & & & \\
\hline $9 \mathrm{H}, \mathrm{CC}$ & 85.70 & 3.13 & & & & & & & & 0.5 & 7.8 & & & & & & & 1.8 & 3.0 & 8.8 & 1.5 & 2.8 & \\
\hline $10 X-2,55-57$ & 87.76 & 3.21 & & & & & & & & & 2.3 & & 0.4 & & & & 1.9 & 11.4 & 12.0 & & 1.3 & 1.5 & \\
\hline $10 X-4,55-57$ & 90.76 & 3.31 & & & & & & & & & 1.0 & & & & & & 3.6 & 17.2 & 2.9 & 0.2 & 1.2 & 1.4 & \\
\hline $10 \mathrm{X}, \mathrm{CC}$ & 95.30 & 3.47 & & & & & & & & & 1.6 & & & & & & & 17.0 & 4.0 & 0.9 & & 0.2 & \\
\hline $11 X-2,55-57$ & 97.36 & 3.54 & & & & 0.4 & & & & & 3.3 & & 0.4 & & & & & 3.5 & 1.8 & 0.2 & 0.7 & 1.3 & 1.3 \\
\hline $11 X-4,55-57$ & 100.36 & 3.59 & & & & & & & & & 1.8 & 0.7 & 0.7 & & & 0.4 & 1.5 & 1.5 & 3.0 & & 1.1 & 1.1 & 1.1 \\
\hline $11 \mathrm{X}, \mathrm{CC}$ & 104.90 & 3.64 & & & & 0.2 & & 0.2 & & & 1.8 & 0.2 & & & & 0.7 & & 0.9 & 2.2 & & 0.7 & 1.1 & 0.2 \\
\hline $12 X-2,55-57$ & 106.96 & 3.66 & 0.7 & & & 0.2 & & & & & 3.9 & & 0.5 & & & 0.2 & 0.2 & 0.2 & 1.8 & & 0.4 & 0.4 & 0.4 \\
\hline $12 \times-4,30-32$ & 109.71 & 3.69 & & & & 0.5 & 0.2 & 1.2 & 0.2 & & 3.5 & 0.5 & 1.5 & 0.3 & & 0.2 & 0.6 & 1.4 & 2.1 & 0.3 & 0.9 & 1.4 & 0.5 \\
\hline $12 \mathrm{X}, \mathrm{CC}$ & 114.50 & 3.75 & & & & & & & & & 0.8 & & & & & & & 3.8 & 1.9 & & & 0.5 & \\
\hline $13 X-2,55-57$ & 116.56 & 3.77 & & & & & & & & & 0.5 & & & 0.2 & & 0.2 & 0.2 & & 1.6 & & & 0.2 & 1.9 \\
\hline $13 \times-4,55-57$ & 119.56 & 3.81 & & & & & & & & & 1.4 & & 0.4 & & & & & 0.2 & 1.6 & 0.2 & & 0.2 & 0.2 \\
\hline $13 \mathrm{X}, \mathrm{CC}$ & 124.20 & 3.86 & & & 0.2 & & & & & & 2.2 & & & & & 0.2 & & 0.7 & 2.6 & 0.4 & 0.7 & & 0.9 \\
\hline $14 X-2,55-57$ & 126.26 & 3.89 & 0.4 & & & & & & & & 1.1 & & & & & 0.4 & & & 2.4 & 0.2 & 0.7 & 0.2 & 0.2 \\
\hline $14 X-4,55-57$ & 129.26 & 3.92 & 1.3 & & & 0.3 & & & & 0.3 & 1.1 & & & & & & & & 1.6 & 0.3 & & 0.3 & \\
\hline $14 \mathrm{X}, \mathrm{CC}$ & 133.90 & 3.97 & 2.6 & & & & & & & 0.2 & 0.0 & & & & & & & & 1.9 & 0.7 & 0.2 & & 1.2 \\
\hline $15 X-2,55-57$ & 135.96 & 4.00 & 20.3 & & & & & & & & 1.0 & & & & & & 0.3 & & 0.8 & & 0.3 & & 2.8 \\
\hline $15 X-4,55-57$ & 138.96 & 4.03 & 0.3 & & 0.3 & & & & & 0.3 & & 0.9 & & & & & & & 2.9 & & 0.3 & 2.9 & \\
\hline $15 \mathrm{X}, \mathrm{CC}$ & 143.50 & 4.08 & 4.9 & & 0.2 & 0.2 & & 0.2 & & & 0.3 & & & & & & & & 1.2 & & 0.5 & 0.5 & 0.7 \\
\hline $16 X-2,55-57$ & 145.56 & 4.11 & 22.5 & & & & & & & 0.2 & & & & & & & 1.1 & & 2.6 & & & 0.5 & \\
\hline $16 \times-4,30-32$ & 148.31 & 4.14 & 0.6 & & & 0.3 & & 0.3 & 0.6 & & 0.9 & & & 0.6 & & & & & 1.4 & 0.9 & & 0.3 & \\
\hline $16 \times-4,55-57$ & 148.56 & 4.14 & 1.7 & & & 1.1 & 0.4 & & 0.9 & & 0.6 & & & & & & & & 2.1 & 2.3 & & 0.4 & \\
\hline $16 \mathrm{X}, \mathrm{CC}$ & 153.10 & 4.19 & 7.1 & & & & 0.2 & & & & 0.4 & & & & & & & & 1.9 & 1.0 & 0.2 & 0.8 & 0.4 \\
\hline $17 X-2,55-57$ & 155.16 & 4.22 & 11.9 & & & & & & & & 2.0 & & & & & & & & 4.5 & & & 0.8 & \\
\hline $17 X-4,55-57$ & 158.16 & 4.25 & 3.2 & & & 0.9 & & & & & & & & & & & & & 3.2 & 0.6 & & 0.9 & 1.4 \\
\hline $17 \mathrm{X}, \mathrm{CC}$ & 162.80 & 4.31 & 2.1 & & 0.4 & 0.1 & & 0.4 & & 0.1 & 0.8 & & & 0.1 & & & & & 1.4 & & & 1.1 & \\
\hline $18 \times-2,55-57$ & 164.86 & 4.33 & 9.0 & & & & & & & & 0.2 & & & & & & & & 0.4 & 0.4 & & 0.2 & \\
\hline $18 X-4,55-57$ & 167.86 & 4.36 & 18.6 & & & & & & & & & & & & & & & & 2.2 & 1.0 & & 3.0 & \\
\hline $18 \mathrm{X}, \mathrm{CC}$ & 172.50 & 4.42 & 33.8 & & & & & & & & 0.2 & & & & & & & & 2.2 & 1.0 & & 1.0 & \\
\hline $19 X-2,55-57$ & 174.56 & 4.44 & 8.9 & & & & & & & & 0.4 & & & & & & & & 3.5 & 2.4 & & 0.4 & \\
\hline
\end{tabular}

Anomalinoides globulosus (Chapman and Parr) = Anomalinoides globulosa Chapman and Parr, 1937, Australasian Antarct. Exped., Sci. Res., ser. C, p. 9, fig. 27.

Astrononion gallowayi Loeblich and Tappan, 1953, Smithsonian Misc. Coll., vol. 121 , p. 90 , pl. 17, figs. 4-7.

Astrononion novozealandicum Cushman and Edwards, 1937, Cushman Lab. Foram. Res., Contr., vol. 13, p. 35, pl. 3, figs. 18a-b.

Baggina sp. 1.

Bolivina alata $($ Seguenza $)=$ Vulvulina alata Seguenza, 1862, Accad. Gioenia Sci. Nat. Atti, ser. 2, vol. 18, p. 115, pl. 2, fig. 5.

Bolivina amygdalaeformis Brady, 1884, Challenger-Exped., Rept. Zool., vol. 9 , pl. 53, figs. 28-29.
Bolivina ordinaria Phleger and Parker $=$ Bolivina simplex Phleger and Parker, 1950, Geol. Soc. Am., Mem., vol. 46, p. 14, pl. 7, figs. 4-6 (emend. Bolivina ordinaria Phleger and Parker, 1952, Cushman, Found. Foram. Res., Contr., vol. 3, pt. 1, p. 14).

Bolivina pygmaea Brady, 1881, Quart. J. Micr. Sci., vol. 21, p. 27.

Bolivina seminuda Cushman, 1911, U.S. Natl. Mus., Bull., vol. 71 (pt. 2), p. 34 , fig. 55 .

Bolivina spathulata (Williamson) $=$ Textularia variabilis var. spathulata Williamson, 1858, Ray Soc., London, p. 76, pl. 6, figs. 164-165.

Bolivina striatula Cushman, 1922, Carnegie Inst. Publ, no. 311, p. 27, pl. 3, fig. 10.

Bolivina sp. 1. 


\begin{tabular}{|c|c|c|c|c|c|c|c|c|c|c|c|c|c|c|c|c|c|c|c|c|c|c|c|}
\hline $\begin{array}{l}\text { Core, section, } \\
\text { interval }(\mathrm{cm})\end{array}$ & $\begin{array}{l}\text { Depth } \\
\text { (mbsf) }\end{array}$ & $\begin{array}{l}\text { Age } \\
\text { (Ma) }\end{array}$ & 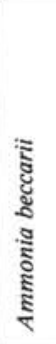 & 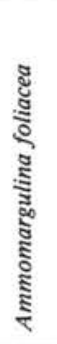 & 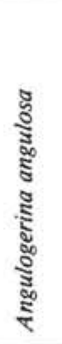 & 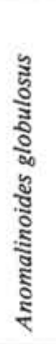 & 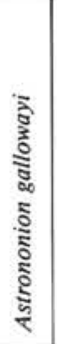 & 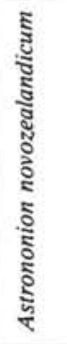 & 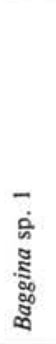 & 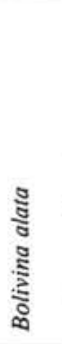 & 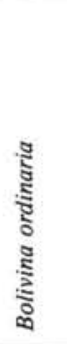 & 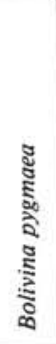 & 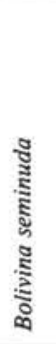 & 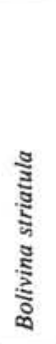 & 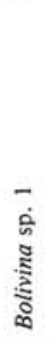 & 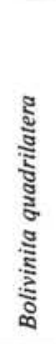 & 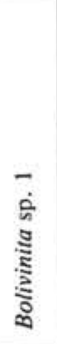 & 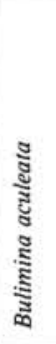 & 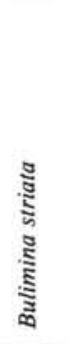 & 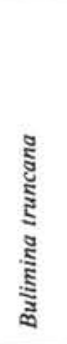 & 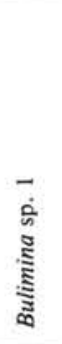 & 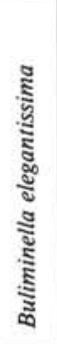 & 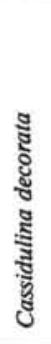 \\
\hline $19 X-4,55-57$ & 177.56 & 4.47 & 1.8 & & & & & & & & 0.9 & & & & & & & & 1.2 & 1.5 & 0.3 & 0.3 & 0.3 \\
\hline $19 \mathrm{X}, \mathrm{CC}$ & 182.20 & 4.53 & 19.9 & & & & & & & & 0.7 & & & 0.4 & & & & & 1.5 & 1.1 & & 0.4 & 0.2 \\
\hline $20 \times-2,55-57$ & 184.26 & 4.55 & 4.6 & & & 0.6 & & & & & & & & & & & & & 1.5 & 0.4 & & & \\
\hline $20 \times-4,55-57$ & 187.26 & 4.59 & 6.2 & & & 0.3 & & & & & 0.3 & & & 0.3 & & & & & 1.9 & 1.5 & & & 0.6 \\
\hline $20 \mathrm{X}, \mathrm{CC}$ & 191.80 & 4.64 & 9.3 & & & 0.3 & & & & & 0.3 & & & & & & & & 1.1 & 0.5 & 0.3 & 0.3 & \\
\hline $21 X-2,55-57$ & 193.86 & 4.66 & 4.1 & & & & & & & & 1.1 & & & & & & & & 1.9 & 0.8 & 0.4 & 0.9 & \\
\hline $21 X-4,55-57$ & 196.86 & 4.70 & 2.9 & & & 0.5 & & & & & 0.5 & & & & & & 0.3 & & 2.4 & 0.3 & & 0.8 & \\
\hline $21 X, C C$ & 201.50 & 4.75 & 3.6 & & & & & & & & 0.4 & & & & & & & & 2.9 & & & 0.4 & \\
\hline $22 \times-2,55-57$ & 203.56 & 4.77 & 3.5 & & & & & & & & & & & & & & & & 1.0 & 0.5 & & & 0.2 \\
\hline $22 X-4,55-57$ & 206.56 & 4.81 & 5.0 & & & & & & & & 1.3 & & 0.2 & & & & & & 0.9 & 0.4 & & & 0.4 \\
\hline $22 \mathrm{X}, \mathrm{CC}$ & 211.10 & 4.86 & 0.4 & & 0.8 & & & & & & 1.8 & & & & & & & & 1.0 & & 0.2 & 2.5 & \\
\hline $23 \mathrm{X}-2,55-57$ & 213.16 & 4.88 & 1.7 & & & 0.3 & & & & & & & & & & & & & 4.2 & & & & \\
\hline $23 X-4,55-57$ & 216.16 & 4.92 & 0.3 & & & 0.3 & & & & & 0.6 & & & & & & & & 2.0 & 0.4 & & 0.3 & \\
\hline $23 \mathrm{X}, \mathrm{CC}$ & 220.80 & 4.97 & 15.2 & & 0.4 & & & & & & 1.4 & & & 1.8 & & & & & 1.1 & & 0.4 & & \\
\hline $24 X-2,55-57$ & 222.86 & 5.00 & & & & 0.2 & & & & & 0.8 & & & & & & 0.2 & & 2.1 & & & 1.1 & \\
\hline $24 \mathrm{X}, \mathrm{CC}$ & 230.50 & 5.08 & & & & & & & & & 0.7 & & & & & & & & 1.8 & & & & \\
\hline $25 X-2,55-57$ & 232.56 & 5.11 & 0.8 & & & & & & & & 1.6 & & & & & & 0.3 & & 2.7 & 0.3 & & 0.3 & \\
\hline $25 \times-4,55-57$ & 235.56 & 5.14 & 0.7 & & & & & 0.5 & & & 1.0 & & & & & & & & 3.2 & & & 0.2 & \\
\hline $25 \mathrm{X}, \mathrm{CC}$ & 240.10 & 5.19 & 0.8 & & & & & & & & 1.9 & & & & & & & & 3.8 & 0.3 & 0.3 & & \\
\hline $26 \times-2,55-57$ & 242.16 & 5.22 & & & & 0.4 & & & & & 3.5 & & & & & & 0.4 & & 1.8 & & & & \\
\hline $26 \times-4,55-57$ & 245.16 & 5.25 & 2.5 & & & 0.6 & & & & & 1.1 & & & & & & & & 2.0 & & & & \\
\hline $26 \mathrm{X}, \mathrm{CC}$ & 249.80 & 5.31 & 0.6 & & 0.4 & 0.4 & & & & & 0.8 & & & 0.2 & & & & & 0.8 & & & & \\
\hline $27 \mathrm{X}-2,55-57$ & 251.86 & 5.33 & 9.9 & & & & & & 0.3 & & 0.5 & & & & & & & & 2.1 & & & & \\
\hline $27 \times-4,55-57$ & 254.86 & 5.36 & 1.4 & & & & & & & 0.3 & 0.9 & & & & & & & & 2.3 & & & & \\
\hline $27 \mathrm{X}, \mathrm{CC}$ & 259.50 & 5.42 & & & & 0.5 & & & & & & & & & & & & & 3.3 & & & & \\
\hline $28 \mathrm{X}-2,55-57$ & 261.56 & 5.44 & & & & & & & & & 1.0 & & & & & & & & 2.4 & & & & \\
\hline $28 X-4,55-57$ & 264.56 & 5.47 & & & & & & & & & 1.3 & & & & & & 0.3 & & 2.5 & & & & \\
\hline $28 \mathrm{X}, \mathrm{CC}$ & 269.10 & 5.53 & & & & & & & & & 1.0 & & & & & & & & 6.5 & 1.3 & & 0.6 & \\
\hline $29 X-2,55-57$ & 271.16 & 5.55 & & & & 0.5 & & & & & 0.9 & & & & & & & & 4.2 & 0.5 & & & \\
\hline $29 \mathrm{X}, \mathrm{CC}$ & 278.80 & 5.64 & & & & & & & & & 0.4 & & & & & & & & 4.3 & 2.0 & & 2.0 & \\
\hline $30 \times-2,55-57$ & 280.86 & 5.66 & & & & & & & & & 1.4 & & & & & & & & 1.4 & 0.7 & & 0.3 & \\
\hline $30 \times-4,55-57$ & 283.86 & 5.70 & & & & & & & & & 0.7 & & & & & & & & 4.2 & 3.6 & & 1.3 & \\
\hline $30 \mathrm{X}, \mathrm{CC}$ & 288.50 & 5.87 & & & & & & & & & 2.4 & & & & & & & & 3.4 & 2.1 & 0.2 & 0.5 & \\
\hline $31 X-2,55-57$ & 290.56 & 5.95 & & & & & & & & & 1.1 & & & & & & & & 3.4 & 0.4 & & 1.1 & 0.4 \\
\hline $31 X-4,55-57$ & 293.56 & 6.06 & & & & & & & & & 0.6 & & & & & & & & 5.3 & 1.1 & & 0.3 & \\
\hline $31 \mathrm{X}, \mathrm{CC}$ & 298.10 & 6.24 & & & & & & & & & & & & 0.4 & & & & & 1.5 & 0.6 & & 0.2 & \\
\hline $32 X-2,55-57$ & 300.16 & 6.32 & & 0.9 & & & & 0.4 & & & & & & & & & & & 1.3 & 0.4 & & & \\
\hline $32 \times-4,55-57$ & 303.16 & 6.43 & & & & & & & & & & & & & & & & & 3.4 & 1.7 & & & \\
\hline $32 \mathrm{X}, \mathrm{CC}$ & 307.80 & 6.61 & & & & & & & & & & & & & & & & & 2.1 & 1.6 & & & \\
\hline $33 \mathrm{X}-1,55-57$ & 308.36 & 6.63 & & & & 0.2 & & & & & & & & & & & & & 1.4 & 1.9 & & & \\
\hline $33 X-4,55-57$ & 312.86 & 6.81 & & & & 0.6 & & & & & 0.3 & & & 0.6 & & & & & 1.5 & 5.1 & & & \\
\hline $33 \mathrm{X}, \mathrm{CC}$ & 317.50 & 6.99 & & & & & & & & & & & & & & & & & 0.4 & 1.1 & & & \\
\hline $34 X-2,55-57$ & 319.56 & 7.07 & & & & 0.6 & & 0.3 & & & 0.3 & & & & & & & & 1.2 & & & 0.6 & \\
\hline $34 X-4,55-57$ & 322.56 & 7.18 & & 2.6 & & 1.1 & 0.3 & & & & & & & 0.3 & & & & & 2.6 & 10.0 & & 5.4 & \\
\hline $34 \mathrm{X}, \mathrm{CC}$ & 327.20 & 7.36 & & & & & & & & & 1.0 & & & & & & & & 1.0 & 1.0 & & & \\
\hline $35 X-2,55-57$ & 329.26 & 7.44 & & & & 0.4 & & & & & & & 1.1 & 0.4 & & & & & 1.4 & 4.0 & & 2.2 & \\
\hline $35 \times-4,55-57$ & 332.26 & 7.55 & & & & & & & 0.4 & & & & & & & & & & 3.2 & 0.7 & & 0.4 & \\
\hline $35 \mathrm{X}, \mathrm{CC}$ & 336.80 & 7.73 & & 2.0 & & 0.2 & & & & & 0.2 & & & & & & & & 1.3 & 9.6 & & 1.8 & \\
\hline $36 \times-2,55-57$ & 338.86 & 7.81 & & & & & & & & & & & & & & & & & 0.7 & 0.7 & & 0.4 & \\
\hline $36 X-4,55-57$ & 341.86 & 7.92 & & & & & & & & & & & & & & & & & 0.9 & & & 0.4 & \\
\hline
\end{tabular}

Bolivina sp. 2.

Bolivinita quadrilatera (Schwager) = Textularia quadrilatera Schwager, 1866, Novara-Exped., Geol. Teil, vol. 2, p. 253, pl. 7, fig. 10.

Bolivinita sp. 1.

Bulimina aculeata d’Orbigny, 1826, Ann. Sci. Nat., ser. 1, vol. 7, p. 269.

Bulimina denudata Cushman and Parker = Bulimina pagoda var. denudata Cushman and Parker, 1928, Cushman Lab. Foram. Res., Contr. vol. 14, p. 57, pl. 10 , figs. 1-2.

Bulimina marginata d'Orbigny, 1826, Ann. Sci. Nat., ser. 1, vol. 7, p. 269 , pl. 12 , figs. $10-12$.

Bulimina mexicana Cushman = Bulimina inflata var. mexicana Cushman, 1922, U.S. Natl. Mus., Bull., vol. 104, p. 95, pl. 21, fig. 2.
Bulimina striata d'Orbigny, 1826, Ann. Sci. Nat., ser. 1, vol. 7, p. 269, pl. 2, fig. 16.

Bulimina subacuminata Cushman and Stewart, in Cushman, Stewart and Stewart, 1930, San Diego Nat. Hist., Trans., vol. 6, no. 2, p. 65, pl. 5, figs. 2-3.

Bulimina subornata Brady, 1884, Challenger-Exped., Rept. Zool., vol. 9, pl. 51 , figs. $6 \mathrm{a}-\mathrm{b}$.

Bulimina truncana Guembel, 1868, K. Akad. Wiss. Wien, Math.-Naturwiss. Kl., Abhandl., p. 644, pl. 2, figs. 77a-b.

Bulimina sp. 1.

Bulimina sp. 3. 
Table 3 (continued).

\begin{tabular}{|c|c|c|c|c|c|c|c|c|c|c|c|c|c|c|c|c|c|c|c|c|c|c|c|}
\hline $\begin{array}{l}\text { Core, section, } \\
\text { interval }(\mathrm{cm})\end{array}$ & $\begin{array}{l}\text { Depth } \\
\text { (mbsf) }\end{array}$ & $\begin{array}{l}\text { Age } \\
(\mathrm{Ma})\end{array}$ & 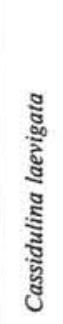 & 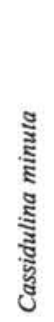 & 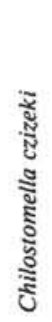 & 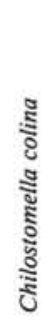 & 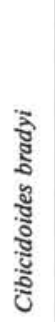 & 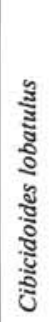 & 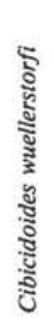 & 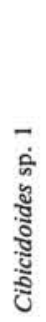 & 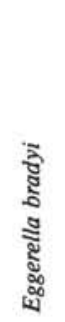 & 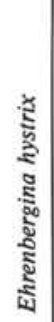 & 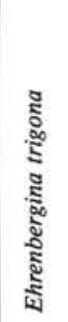 & 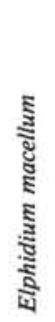 & 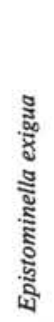 & 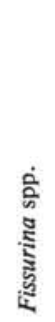 & 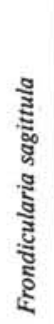 & 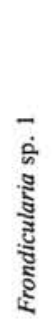 & 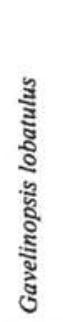 & 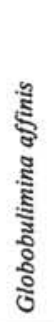 & 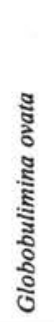 & 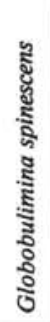 & 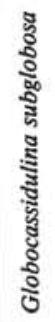 \\
\hline $1 \mathrm{H}-2,55-57$ & 2.06 & 0.05 & 12.8 & & 0.6 & 0.9 & & & 1.5 & & & & 13.7 & & 0.3 & 1.8 & & & 2.7 & & & & 1.8 \\
\hline $1 \mathrm{H}-4,55-57$ & 5.06 & 0.12 & 13.2 & 1.3 & 0.8 & 0.3 & 0.5 & 0.5 & 1.1 & & 1.6 & & 5.7 & & 1.6 & 0.8 & & & 4.3 & & & & 7.0 \\
\hline $1 \mathrm{H}, \mathrm{CC}$ & 9.60 & 0.23 & 6.1 & 0.9 & 0.6 & 0.3 & 2.0 & & & & & & 5.5 & & & 0.6 & & & 8.1 & & & & 7.3 \\
\hline $2 \mathrm{H}-2,55-57$ & 11.66 & 0.28 & 11.0 & & 0.5 & 1.0 & 5.4 & 0.5 & 1.5 & & & & 2.0 & & 0.8 & 1.5 & & & 4.3 & 0.3 & & & 2.8 \\
\hline $2 \mathrm{H}-4,55-57$ & 14.66 & 0.35 & 14.9 & 0.2 & 0.3 & & 0.5 & 0.8 & 1.6 & & 0.5 & & 3.6 & & 0.6 & 0.6 & & & 0.8 & & & & 5.5 \\
\hline $2 \mathrm{H}, \mathrm{CC}$ & 19.10 & 0.45 & 49.0 & 0.2 & & & 0.3 & 1.8 & 5.0 & & & & 0.8 & & 0.2 & 0.3 & & & 4.0 & & & & 1.4 \\
\hline $3 \mathrm{H}, \mathrm{CC}$ & 28.50 & 0.67 & 20.4 & 1.3 & 0.3 & & 1.1 & & 0.8 & & & & 1.3 & & 0.8 & 0.8 & & & 6.1 & & & & 5.0 \\
\hline $4 \mathrm{H}-4,55-57$ & 33.56 & 0.79 & 7.7 & 0.3 & 0.8 & & 1.1 & & 0.3 & & 1.7 & & 0.6 & & 0.3 & 0.3 & & & 7.2 & 0.6 & & & 3.9 \\
\hline $4 \mathrm{H}, \mathrm{CC}$ & 38.00 & 0.90 & 32.3 & 0.7 & & & 1.0 & & 2.2 & & 1.0 & & 3.2 & & & 0.2 & & & 0.7 & & & & 5.2 \\
\hline $5 \mathrm{H}-2,55-57$ & 40.06 & 0.95 & 23.1 & 0.0 & & & & & 0.4 & & 0.4 & & 0.4 & & & & & & 0.8 & & & & 5.0 \\
\hline $5 \mathrm{H}-4,55-57$ & 43.06 & 1.04 & 7.8 & 1.4 & & 0.4 & 0.7 & & & & 1.1 & & 1.8 & & & 1.4 & & & 0.4 & & & & 5.3 \\
\hline $5 \mathrm{H}, \mathrm{CC}$ & 47.50 & 1.21 & 4.5 & 0.8 & & & 0.9 & & 0.2 & & & & 0.3 & & & 0.6 & & & 1.2 & & & & 7.7 \\
\hline $6 \mathrm{H}-2,55-57$ & 49.56 & 1.29 & 28.4 & 0.2 & & & 0.6 & 0.2 & & & 0.8 & & 0.6 & & & 0.8 & & & 1.6 & & & 0.4 & 1.0 \\
\hline $6 \mathrm{H}, \mathrm{CC}$ & 57.00 & 1.58 & 22.1 & 1.6 & & & 1.3 & & 0.5 & & 1.0 & & & & & 1.8 & & & 11.7 & & & & 0.8 \\
\hline $7 \mathrm{H}-3,30-32$ & 60.31 & 1.71 & 17.8 & 0.5 & 0.3 & & 1.3 & 0.3 & & & 1.0 & & & & & 0.5 & 0.3 & & 3.1 & & & & \\
\hline $7 \mathrm{H}-5,100-102$ & 64.01 & 1.85 & 16.9 & & & & 1.6 & & & & & & 0.4 & & & 1.6 & 0.8 & 0.4 & 0.8 & & & 0.4 & 0.4 \\
\hline $7 \mathrm{H}, \mathrm{CC}$ & 66.50 & 1.98 & 3.1 & 0.4 & & & 0.7 & & & & & & & & & 2.6 & & & 8.1 & & & & 3.3 \\
\hline $8 \mathrm{H}-4,55-57$ & 71.56 & 2.35 & 22.5 & & & & & & 0.3 & & & & & & & 1.6 & 0.5 & & 0.8 & 0.3 & & & 11.1 \\
\hline $8 \mathrm{H}-6,55-57$ & 74.56 & 2.56 & 3.1 & & & & & & & & & & & & & 0.9 & 2.8 & 0.6 & 0.6 & 0.3 & & 0.3 & 9.3 \\
\hline $8 \mathrm{H}, \mathrm{CC}$ & 76.10 & 2.67 & 4.2 & & & & & & 0.2 & & & & & & 0.2 & 4.2 & & & 0.2 & & & & 6.9 \\
\hline $9 \mathrm{H}, \mathrm{CC}$ & 85.70 & 3.13 & 1.0 & & & & & & 1.3 & & & & 2.5 & & & 1.5 & & & & & & & 5.0 \\
\hline $10 X-2,55-57$ & 87.76 & 3.21 & 5.7 & & & & & & 0.2 & & & & 5.7 & & & 0.6 & 1.3 & & 2.1 & & 0.2 & & 3.8 \\
\hline $10 X-4,55-57$ & 90.76 & 3.31 & 6.2 & & & & 0.2 & & 0.7 & & 0.7 & & & & & 0.5 & 1.2 & & 1.2 & 0.2 & & & 3.6 \\
\hline $10 \mathrm{X}, \mathrm{CC}$ & 95.30 & 3.47 & 7.2 & & & & 0.5 & & 2.1 & & & & 6.0 & & & 1.4 & & & & & & & 7.9 \\
\hline $11 X-2,55-57$ & 97.36 & 3.54 & 7.5 & 0.2 & & & 0.4 & & 0.2 & & 0.2 & & 1.3 & & 0.2 & 0.9 & 1.8 & & 0.9 & & & 0.4 & 4.0 \\
\hline $11 X-4,55-57$ & 100.36 & 3.59 & 5.2 & & & & & & & & 0.4 & & 0.4 & & & 2.2 & 0.7 & & 1.8 & & & 0.4 & 5.5 \\
\hline $11 \mathrm{X}, \mathrm{CC}$ & 104.90 & 3.64 & 5.1 & 0.4 & & & 1.3 & & 0.2 & & 0.9 & & 8.9 & & & 2.0 & & & 0.2 & & & & 10.3 \\
\hline $12 X-2,55-57$ & 106.96 & 3.66 & 22.4 & & & & & & 0.9 & & 2.7 & & 0.4 & & & 3.0 & 0.4 & & 0.2 & 0.2 & 0.2 & 0.4 & 3.0 \\
\hline $12 X-4,30-32$ & 109.71 & 3.69 & 5.4 & & & & 3.8 & & & & 1.1 & & 2.1 & & 0.6 & 1.8 & 1.5 & & 0.5 & 0.2 & & 0.2 & 2.4 \\
\hline $12 \mathrm{X}, \mathrm{CC}$ & 114.50 & 3.75 & 5.4 & & & & & & & & 1.1 & & 11.3 & & 0.5 & 2.4 & & & 0.8 & & & & 3.2 \\
\hline $13 X-2,55-57$ & 116.56 & 3.77 & 5.4 & 0.2 & & & 1.9 & & 0.5 & & 0.2 & & 4.9 & & & 1.9 & 1.4 & & 0.5 & 1.2 & & 0.5 & 6.5 \\
\hline $13 X-4,55-57$ & 119.56 & 3.81 & 3.6 & & & & 4.2 & & 1.2 & & 0.8 & & 16.2 & & 0.2 & 1.8 & 1.4 & & 0.6 & 0.6 & 0.2 & 0.2 & 7.3 \\
\hline $13 \mathrm{X}, \mathrm{CC}$ & 124.20 & 3.86 & 0.7 & & & & & & 0.9 & & 0.9 & & 11.9 & & 1.1 & 2.0 & & & 0.4 & & & & 2.6 \\
\hline $14 X-2,55-57$ & 126.26 & 3,89 & 1.7 & & & & 2.2 & & 1.1 & & 2.0 & & 14.1 & 0.2 & 0.2 & 2.0 & 3.3 & 0.2 & 0.9 & 0.9 & 0.2 & 1.1 & 1.5 \\
\hline $14 X-4,55-57$ & 129.26 & 3.92 & 1.1 & 0.5 & 0.0 & & 3.5 & 0.5 & 1.1 & & 4.0 & 0.3 & 10.9 & & 0.3 & 2.4 & 1.6 & & & & & & 5.1 \\
\hline $14 \mathrm{X}, \mathrm{CC}$ & 133.90 & 3.97 & 2.1 & & 0.5 & & & & 0.2 & & 1.9 & & 4.4 & & 1.2 & 5.1 & 1.6 & & 0.7 & & & & 2.8 \\
\hline $15 X-2,55-57$ & 135.96 & 4.00 & 1.3 & & & & 1.5 & & 0.8 & & 0.5 & & 4.6 & & & 1.3 & 2.0 & 0.3 & & & & 0.5 & 3.6 \\
\hline $15 \times-4,55-57$ & 138.96 & 4.03 & 0.3 & & 0.3 & & & & 2.3 & & & & & & 0.3 & 2.0 & 3.7 & & & 0.6 & & 0.3 & 3.4 \\
\hline $15 \mathrm{X}, \mathrm{CC}$ & 143.50 & 4.08 & 0.2 & 1.2 & 0.2 & & & & 1.0 & & 1.2 & & 1.4 & & 0.2 & 1.7 & 3.6 & & 0.2 & & & 0.3 & 1.4 \\
\hline $16 \times-2,55-57$ & 145.56 & 4.11 & 0.2 & 0.4 & & & & & & & 0.9 & & 3.8 & & & 2.7 & 0.7 & & & 0.9 & & & 1.1 \\
\hline $16 X-4,30-32$ & 148.31 & 4.14 & & 1.7 & & & 1.7 & 0.3 & 1.1 & & 3.7 & & 6.0 & & & 0.9 & 1.1 & & 0.3 & & & & 10.0 \\
\hline $16 \times-4,55-57$ & 148.56 & 4.14 & & 0.9 & & & 2.1 & 0.6 & 0.9 & & 12.3 & & 2.3 & & 0.4 & 1.1 & 1.7 & & 1.3 & & & & 14.5 \\
\hline $16 \mathrm{X}, \mathrm{CC}$ & 153.10 & 4.19 & 0.2 & 0.6 & 0.4 & & & & 0.8 & & 0.8 & & 2.9 & & 1.2 & 1.9 & 1.0 & & 0.2 & & & & 2.3 \\
\hline $17 X-2,55-57$ & 155.16 & 4.22 & & & & & 0.5 & & 0.5 & & 1.8 & & 6.8 & & 2.5 & 2.8 & 0.8 & 0.3 & & & & 0.5 & 1.3 \\
\hline $17 \times-4,55-57$ & 158.16 & 4.25 & & 0.6 & & & 4.6 & & 1.2 & & 2.3 & & 4.1 & & 2.0 & 2.3 & 2.6 & & 1.2 & & & 0.3 & 7.0 \\
\hline $17 \mathrm{X}, \mathrm{CC}$ & 162.80 & 4.31 & 0.6 & 0.4 & & & & & 0.1 & & 2.2 & & 1.1 & & 2.1 & 1.5 & 2.4 & & & & & & 2.7 \\
\hline $18 X-2,55-57$ & 164.86 & 4.33 & 0.2 & 0.2 & & & & & 1.1 & & 0.2 & & 0.2 & & 0.2 & 1.1 & 3.5 & & & 0.9 & & & 2.6 \\
\hline $18 \times-4,55-57$ & 167.86 & 4.36 & 0.2 & 0.5 & 0.2 & & & & 0.2 & & 0.2 & & 0.2 & & & 2.2 & 5.7 & 0.2 & & 0.5 & 0.5 & & 1.5 \\
\hline $18 \mathrm{X}, \mathrm{CC}$ & 172.50 & 4.42 & 0.6 & & & & & & & & 0.7 & & & & 0.2 & 2.9 & 2.2 & & & & & & 0.2 \\
\hline $19 X-2,55-57$ & 174.56 & 4.44 & 0.4 & 1.1 & & & 0.2 & & 0.2 & & 3.1 & & 2.4 & & & 1.5 & 1.7 & 0.9 & 0.4 & 0.4 & & 0.2 & 2.4 \\
\hline
\end{tabular}

Buliminella elegantissima (d'Orbigny) = Bulimina elegantissima d'Orbigny, 1839, Voy. Am. Merid., vol. 5, pt. 5, p. 51, pl. 7, figs. 13-14.

Cancris oblongus $($ Williamson) $=$ Rotalina oblonga Williamson, 1858, Ray Soc., London, p. 51, pl. 4, figs. 98-100.

Cancris sp. 1.

Cassidulina decorata Sidebottom, 1910, Quekett Microsc. Club, J., ser. 2, vol. 11, p. 107, pl. 4, figs. 2a-c.

Cassidulina laevigata d'Orbigny, 1826, Ann. Sci. Nat., ser. 1, vol. 7, p. 282 , pl. 15 , figs. $4-5$.

Cassidulina minuta Cushman, 1933, Cushman Lab. Foram. Res., Contr., vol. 9 , pt. 4 , p. 92 , pl. 10 , fig. 3 .

Cassidulina sp. 1.

Chilostomella czizeki Reuss, 1850, K. Akad. Wiss. Wien, Math-Naturwiss. Kl., Denkschr., vol. 1, p. 380 , pl. 48, figs. 13a-d.

Chilostomella oolina Schwager, 1878, Boll. Reale Comitato Geol. d'Italia, vol. 9, p. 527, pl. 1, fig. 16.

Cibicidoides bradyi (Trauth) = Truncatulina dutemplei Brady, 1884, Challenger-Exped., Rept. Zool., vol. 9, p. 665, pl. 95, fig. 5. = Truncatulina bradyi Trauth, 1918, K. Akad. Wiss. Wien, Math-Naturwiss. Kl., Denkschr., vol. 95, p. 235.

Cibicidoides lobatulus (Walker and Jacob) $=$ Nautilus lobatulus Walker and Jacob, 1798, in Kanmacher, 1798, p. 642, pl. 14, fig. 36.

Cibididoides wuellerstorfi $($ Schwager) $=$ Anomalina wuellerstorfi Schwager, 1866, Novara-Exped., Geol. Teil, vol. 2, p. 258, pl. 7, figs. 105107.

Cibicidoides sp. 1.

Cibicidoides sp. 2.

Cibicidoides sp. 3. 


\begin{tabular}{|c|c|c|c|c|c|c|c|c|c|c|c|c|c|c|c|c|c|c|c|c|c|c|c|}
\hline $\begin{array}{l}\text { Core, section, } \\
\text { interval }(\mathrm{cm})\end{array}$ & $\begin{array}{l}\text { Depth } \\
\text { (mbsf) }\end{array}$ & $\begin{array}{l}\text { Age } \\
\text { (Ma) }\end{array}$ & 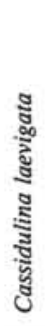 & 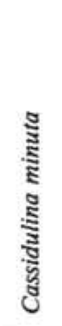 & 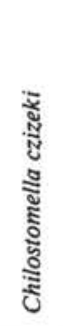 & 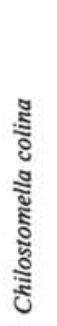 & 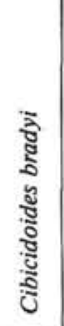 & 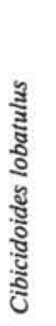 & 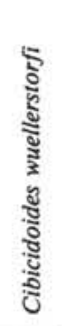 & 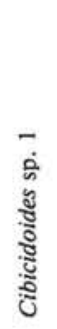 & 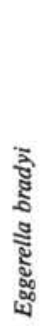 & 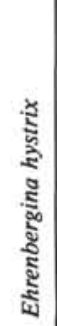 & 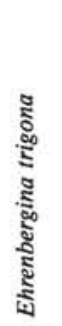 & 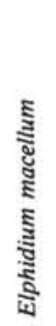 & 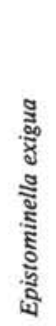 & 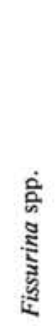 & 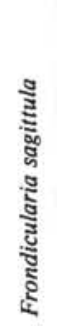 & 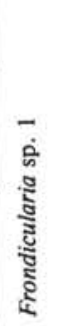 & 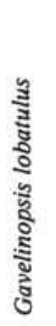 & 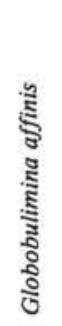 & 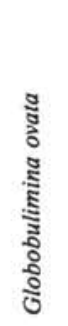 & 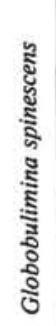 & 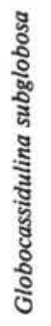 \\
\hline $19 X-4,55-57$ & 177.56 & 4.47 & & 1.8 & & & 1.5 & & 0.6 & & 1.8 & & 4.2 & & & 2.7 & 3.6 & & & 0.3 & & & 0.9 \\
\hline $19 \mathrm{X}, \mathrm{CC}$ & 182.20 & 4.53 & 0.2 & 0.2 & & & & & 0.2 & & 1.1 & & 0.4 & & & 1.8 & 7.2 & & 0.4 & & & & 0.2 \\
\hline $20 X-2,55-57$ & 184.26 & 4.55 & 0.4 & 0.2 & 0.4 & & 0.2 & & 0.6 & & 1.3 & & 0.8 & & & 0.6 & 6.5 & & & & & & \\
\hline $20 \times-4,55-57$ & 187.26 & 4.59 & & & & & 2.5 & & 0.3 & & 1.2 & & 1.2 & & & 0.6 & 3.1 & & & & & & \\
\hline $20 \mathrm{X}, \mathrm{CC}$ & 191.80 & 4.64 & & & 0.3 & & & & & & & & 2.6 & & 0.3 & 1.1 & 4.2 & & & & & & 2.6 \\
\hline $21 X-2,55-57$ & 193.86 & 4.66 & & & 0.2 & & & & & & 0.4 & & 4.7 & & & 2.8 & 1.5 & 0.2 & 0.4 & 0.2 & & & 1.3 \\
\hline $21 X-4,55-57$ & 196.86 & 4.70 & 0.3 & 0.3 & & & 1.1 & & 0.8 & & 0.3 & & 2.4 & & & 2.6 & 2.9 & & 0.3 & & & & 0.3 \\
\hline $21 \mathrm{X}, \mathrm{CC}$ & 201.50 & 4.75 & & & & & & & 1.1 & & & & 4.3 & & & 3.2 & 3.2 & & & & & & 2.1 \\
\hline $22 X-2,55-57$ & 203.56 & 4.77 & & 0.2 & & & 0.7 & & 4.0 & & 0.2 & & 1.5 & & & 3.0 & 3.0 & & & 0.2 & & & \\
\hline $22 X-4,55-57$ & 206.56 & 4.81 & 0.2 & 0.2 & & & 0.4 & & 3.2 & & 1.1 & & 0.2 & & & 3.7 & 2.8 & 0.4 & & & & & 0.9 \\
\hline $22 \mathrm{X}, \mathrm{CC}$ & 211.10 & 4.86 & & & & & & & 2.5 & & 0.6 & & 2.5 & & & 2.0 & 4.3 & & & & & & 1.2 \\
\hline $23 X-2,55-57$ & 213.16 & 4.88 & & & & & 0.6 & & 5.6 & & & & 9.0 & & & 2.8 & 2.8 & & & & & & \\
\hline $23 \mathrm{X}-4,55-57$ & 216.16 & 4.92 & & & & & 1.1 & & 7.3 & & 0.6 & & 0.6 & & & 2.2 & 3.2 & & & 0.3 & & & 0.4 \\
\hline $23 \mathrm{X}, \mathrm{CC}$ & 220.80 & 4.97 & 0.4 & & & & & & & & & & 2.5 & & & 4.0 & 2.9 & & & & & & 0.4 \\
\hline $24 \mathrm{X}-2,55-57$ & 222.86 & 5.00 & & & & & 1.4 & & 9.5 & & 0.2 & & 2.1 & & 0.2 & 1.8 & 1.9 & & & & & & 1.1 \\
\hline $24 \mathrm{X}, \mathrm{CC}$ & 230.50 & 5.08 & 0.7 & & & & & & 7.2 & & 1.1 & & 0.4 & & & 4.7 & 1.8 & & & & & & 3.6 \\
\hline $25 \mathrm{X}-2,55-57$ & 232.56 & 5.11 & & & & & & & 0.3 & & 0.5 & & & & & 3.6 & 1.1 & 0.3 & 0.8 & 1.1 & 0.8 & & 3.8 \\
\hline $25 X-4,55-57$ & 235.56 & 5.14 & & & & & 1.2 & & 0.5 & & 0.7 & & & & 0.2 & 2.2 & 2.0 & & & & 0.2 & & 5.2 \\
\hline $25 \mathrm{X}, \mathrm{CC}$ & 240.10 & 5.19 & & & & & & & & & 0.3 & & & & & 1.6 & 2.2 & & & & & & 2.4 \\
\hline $26 X-2,55-57$ & 242.16 & 5.22 & 0.4 & 0.7 & 0.7 & & 2.1 & & 1.4 & & 2.8 & & 1.1 & & 0.7 & 4.9 & 2.8 & & & 0.4 & 0.4 & & 1.8 \\
\hline $26 \times-4,55-57$ & 245.16 & 5.25 & 0.3 & & & & 0.3 & & 1.1 & & 2.0 & & & & & 0.8 & 2.3 & & & 1.4 & 0.3 & 0.3 & 2.0 \\
\hline $26 \mathrm{X}, \mathrm{CC}$ & 249.80 & 5.31 & & 0.2 & & & & & 0.4 & & 1.3 & & & & & 3.1 & 2.5 & & & & & & 5.4 \\
\hline $27 \mathrm{X}-2,55-57$ & 251.86 & 5.33 & 2.3 & 0.3 & & & 0.5 & & 0.5 & & 0.8 & & & & & 2.1 & 0.3 & & & 0.5 & 0.3 & & 0.8 \\
\hline $27 \times-4,55-57$ & 254.86 & 5.36 & & 0.9 & & & 2.9 & & 5.5 & & 0.3 & & & & & 1.1 & 2.6 & & & & & & 3.2 \\
\hline $27 \mathrm{X}, \mathrm{CC}$ & 259.50 & 5.42 & & 1.3 & & & & & & & 1.0 & & & & 0.5 & 2.8 & 1.8 & & & & & & 4.6 \\
\hline $28 \mathrm{X}-2,55-57$ & 261.56 & 5.44 & & 0.2 & & & 0.7 & & 1.5 & & 0.5 & & & & & 0.5 & 0.7 & & & 0.2 & & & 2.7 \\
\hline $28 \mathrm{X}-4,55-57$ & 264.56 & 5.47 & & & & & 0.3 & & 0.3 & & 1.6 & 0.9 & & & 0.6 & 4.1 & 3.4 & & 0.3 & & 0.3 & & 1.3 \\
\hline $28 \mathrm{X}, \mathrm{CC}$ & 269.10 & 5.53 & & & 0.3 & & & & & & 1.0 & & & & & 2.3 & 1.9 & & & & & & 0.6 \\
\hline $29 \mathrm{X}-2,55-57$ & 271.16 & 5.55 & & & & & 2.5 & & 0.5 & & 0.5 & 0.2 & 0.2 & & 1.4 & 1.4 & 0.5 & 0.2 & & 0.7 & & 0.2 & 1.4 \\
\hline $29 \mathrm{X}, \mathrm{CC}$ & 278.80 & 5.64 & & & & & & & 0.4 & & 2.0 & & & & 0.8 & 2.0 & 2.0 & & & & & & \\
\hline $30 \times-2,55-57$ & 280.86 & 5.66 & & & & & 4.4 & & 1.4 & & 1.7 & & & & 2.0 & 2.4 & 4.7 & & & & & 0.3 & 1.0 \\
\hline $30 \times-4,55-57$ & 283.86 & 5.70 & 0.3 & 0.3 & & & 0.7 & & 0.3 & & 2.0 & & & & 0.3 & 2.9 & 8.5 & & 0.3 & & & & 0.7 \\
\hline $30 \mathrm{X}, \mathrm{CC}$ & 288.50 & 5.87 & & & & & & & & & 0.2 & & & & & 2.1 & 2.8 & & & & & & 1.3 \\
\hline $31 X-2,55-57$ & 290.56 & 5.95 & & & & & 1.5 & & 0.4 & & 3.4 & & & & 0.4 & 3.0 & 2.3 & 0.4 & & & & & 1.5 \\
\hline $31 \times-4,55-57$ & 293.56 & 6.06 & & & & & 1.1 & & 0.6 & & 0.0 & & & & & 2.2 & 1.7 & & & & & & \\
\hline $31 \mathrm{X}, \mathrm{CC}$ & 298.10 & 6.24 & & & & & & & 8.6 & & 0.2 & & & & & 2.1 & 0.8 & 0.4 & & & & & 2.1 \\
\hline $32 X-2,55-57$ & 300.16 & 6.32 & & & & & 2.1 & & 0.4 & & & & 0.9 & & 0.4 & 2.1 & 2.1 & 0.4 & & & & & 1.3 \\
\hline $32 X-4,55-57$ & 303.16 & 6.43 & & & & & 0.6 & & 0.6 & & 0.6 & & & & & 0.6 & 6.3 & 1.1 & & & 0.6 & & \\
\hline $32 \mathrm{X}, \mathrm{CC}$ & 307.80 & 6.61 & & & & & & & & & 0.3 & & & & & 1.3 & 4.0 & & & & & & \\
\hline $33 X-1,55-57$ & 308.36 & 6.63 & & & & & 4.7 & & & & 1.2 & & 0.2 & & 0.5 & 2.3 & 3.7 & 1.4 & & 0.2 & & & 3.0 \\
\hline $33 \times-4,55-57$ & 312.86 & 6.81 & 0.3 & & & & 2.1 & & & & 1.2 & & 0.3 & & 0.6 & 1.8 & 3.3 & 0.3 & & 0.3 & & & \\
\hline $33 \mathrm{X}, \mathrm{CC}$ & 317.50 & 6.99 & & & & & & & & & & & & & & 1.4 & 6.5 & 1.1 & & 0.4 & 0.4 & & 0.7 \\
\hline $34 X-2,55-57$ & 319.56 & 7.07 & & & & & 8.7 & & & & 1.8 & & 1.2 & & & 0.9 & 3.6 & 0.3 & & 0.6 & & & 1.5 \\
\hline $34 \times-4,55-57$ & 322.56 & 7.18 & & & & & 1.1 & & 0.6 & & 0.3 & & 0.3 & & & 1.4 & 2.3 & & & 0.6 & & & 2.6 \\
\hline $34 \mathrm{X}, \mathrm{CC}$ & 327.20 & 7.36 & & & & & 9.4 & & & & 1.0 & & & & & & 6.3 & & & 1.0 & & & \\
\hline $35 X-2,55-57$ & 329.26 & 7.44 & & & & & 12.3 & & 0.7 & 3.2 & 3.3 & & 0.7 & & 1.1 & 4.0 & 5.1 & & & & & & 0.4 \\
\hline $35 X-4,55-57$ & 332.26 & 7.55 & & & & & 4.6 & & 0.4 & 1.4 & 2.5 & & & & & 0.4 & 2.1 & 0.7 & & & & & 0.7 \\
\hline $35 \mathrm{X}, \mathrm{CC}$ & 336.80 & 7.73 & & & & & 4.3 & & 0.2 & & 1.1 & & 0.2 & & & 1.6 & 1.6 & & & & & & 0.7 \\
\hline $36 X-2,55-57$ & 338.86 & 7.81 & & & & & 13.3 & & 0.7 & & & & & & & 1.1 & 1.9 & & & & & & \\
\hline $36 \times-4,55-57$ & 341.86 & 7.92 & & & & & 11.7 & & 0.4 & & 0.9 & & 0.4 & & & & 0.4 & & & & & & \\
\hline
\end{tabular}

Eggerella bradyi $($ Cushman $)=$ Verneuilina bradyi Cushman, 1911, U.S. Natl. Mus., Bull., vol. 71 (pt. 2), p. 54, text-fig. 87.

Ehrenbergina hystrix Brady, 1884, Challenger-Exped., Rept. Zool., vol. 9, p. 435 , pl. 55, figs. 8-11.

Ehrenbergina trigona Goës = Ehrenberina serrata var. trigona Goës, 1896, Harvard Coll., Mus. Comparative Zool., Bull., vol. 29, no. 1 , p. 49 , pl. 6 , figs. $183-184$.

Elphidium excavatum (Terquem) $=$ Polystomella excavata Terquem, 1876, Soc. Dunkerquoise, Mem., vol. 19, p. 429, pl. 2, figs. 2a-d.

Elphidium macellum (Fichtel and Moll) $=$ Nautilus macellus Fichtel and Moll, 1798, Testacea Microsc., p. 6, var. $\alpha$, pl. 10, figs. e-g; var. $\beta$, pl. 10 , figs. $\mathrm{h}-\mathrm{k}$.
Epistominella exigua $($ Brady) $=$ Pulvinulina exigua Brady, 1884, Challenger-Exped., Rept., Zool., vol. 9, p. 696, pl. 103, figs. 13a-c.

Fissurina spp.

Florilus sp. 2.

Florilus sp. 3.

Frondicularia sagittula Van den Broeck $=$ Frondicularia alata var. sagittula Van den Broeck, 1876, Ann. Soc. Belge Micr., vol. 2, p. 113, pl. 2, figs. 12, 14 .

Frondicularia sp. 1

Gavelinopsis lobatulus (Parr) = Discorbis lobatulus Parr, 1950, Brit. New Zeal. Antarct. Res. Exped., Rept., ser. B, vol. 5, (pt. 6), p. 354, pl. 13 , figs. $23-25$. 
Table 3 (continued).

\begin{tabular}{|c|c|c|c|c|c|c|c|c|c|c|c|c|c|c|c|c|c|c|c|c|c|c|}
\hline $\begin{array}{l}\text { Core, section, } \\
\text { interval }(\mathrm{cm})\end{array}$ & $\begin{array}{l}\text { Depth } \\
\text { (mbsf) }\end{array}$ & $\begin{array}{l}\text { Age } \\
(\mathrm{Ma})\end{array}$ & 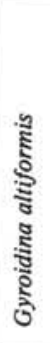 & 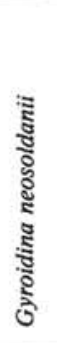 & 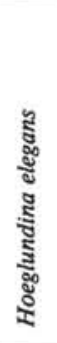 & 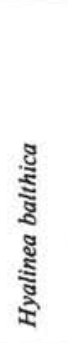 & 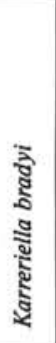 & 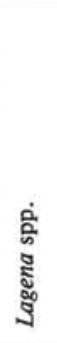 & 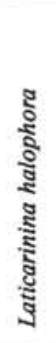 & 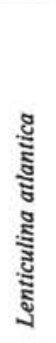 & 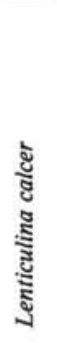 & 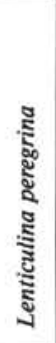 & 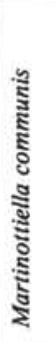 & 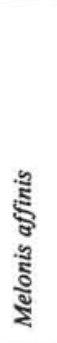 & 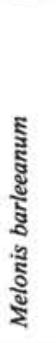 & 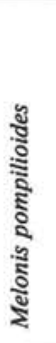 & 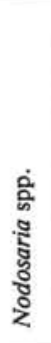 & 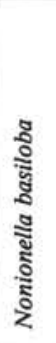 & 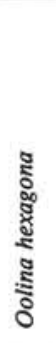 & 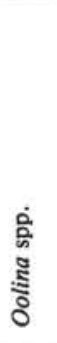 & 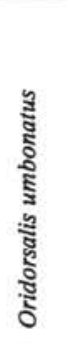 & 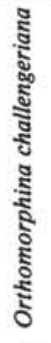 \\
\hline $1 \mathrm{H}-2,55-57$ & 2.06 & 0.05 & & & & & & & & & & & & & 0.6 & & & 1.8 & 0.3 & & 2.4 & \\
\hline $1 \mathrm{H}-4,55-57$ & 5.06 & 0.12 & & & 1.6 & & 0.3 & 0.5 & & & & & & & & & & 0.3 & & 0.3 & 1.6 & \\
\hline $1 \mathrm{H}, \mathrm{CC}$ & 9.60 & 0.23 & & & 9.3 & & & 0.6 & & 0.3 & 0.3 & & 0.3 & 0.6 & 2.9 & 0.3 & & 0.8 & 0.3 & & 1.2 & \\
\hline $2 \mathrm{H}-2,55-57$ & 11.66 & 0.28 & & & 1.8 & 0.5 & 2.0 & & & 1.5 & 0.3 & 0.8 & 0.3 & & 3.6 & & & 0.3 & & 1.0 & 2.6 & \\
\hline $2 \mathrm{H}-4,55-57$ & 14.66 & 0.35 & & & 1.4 & & & 0.2 & 0.3 & & & & & 0.3 & 4.3 & & & & 0.3 & & 4.7 & \\
\hline $2 \mathrm{H}, \mathrm{CC}$ & 19.10 & 0.45 & & & & & & 0.2 & & & & & & & 0.5 & & & 1.3 & 0.2 & & 2.7 & \\
\hline $3 \mathrm{H}, \mathrm{CC}$ & 28.50 & 0.67 & & & 2.1 & 1.3 & 0.3 & 1.1 & & 0.3 & & & & & 0.5 & & & 0.5 & & 0.8 & 4.2 & \\
\hline $4 \mathrm{H}-4,55-57$ & 33.56 & 0.79 & & & 0.3 & 3.9 & 0.3 & & & & & & & & 1.1 & & & 0.8 & 0.3 & & 1.1 & \\
\hline $4 \mathrm{H}, \mathrm{CC}$ & 38.00 & 0.90 & & & & & 0.2 & 0.2 & 0.5 & & & & & 0.2 & 0.5 & & & & & & 2.7 & \\
\hline $5 \mathrm{H}-2,55-57$ & 40.06 & 0.95 & & & & & & & & & & 0.4 & & & & & & & & & 2.3 & \\
\hline $5 \mathrm{H}-4,55-57$ & 43.06 & 1.04 & & & & & & & 0.4 & & & & & & & & & & & 0.4 & 4.3 & 0.4 \\
\hline $5 \mathrm{H}, \mathrm{CC}$ & 47.50 & 1.21 & & & & 12.3 & & 0.8 & 0.3 & 0.3 & & & & & 0.2 & & & 0.2 & 0.2 & 1.1 & 6.5 & \\
\hline $6 \mathrm{H}-2,55-57$ & 49.56 & 1.29 & & & 0.4 & 5.7 & & & & & & 0.2 & & & 1.0 & & & & & & 3.3 & \\
\hline $6 \mathrm{H}, \mathrm{CC}$ & 57.00 & 1.58 & & & & & & 0.3 & & & & & & 2.1 & 0.8 & & & & 0.3 & & 7.0 & \\
\hline $7 \mathrm{H}-3,30-32$ & 60.31 & 1.71 & & & 0.5 & 2.8 & & & 0.3 & & & & & 0.5 & 1.3 & & & & 0.3 & & 3.6 & 0.3 \\
\hline $7 \mathrm{H}-5,100-102$ & 64.01 & 1.85 & & & 0.4 & & & & 0.4 & & & & & 0.8 & 1.2 & & & & 0.8 & 1.2 & 5.2 & \\
\hline $7 \mathrm{H}, \mathrm{CC}$ & 66.50 & 1.98 & & & 0.9 & & & 1.7 & & 0.2 & & & & 0.7 & 0.4 & & & 0.2 & & & 3.5 & \\
\hline $8 \mathrm{H}-4,55-57$ & 71.56 & 2.35 & & & & 0.3 & & & & & & & & & & & & & 1.3 & 0.5 & 1.9 & \\
\hline $8 \mathrm{H}-6,55-57$ & 74.56 & 2.56 & & & & 5.0 & & & & & & & & & & & & & 1.9 & 0.6 & 8.7 & \\
\hline $8 \mathrm{H}, \mathrm{CC}$ & 76.10 & 2.67 & & & 0.2 & 1.9 & & 1.9 & & 0.7 & & & & 0.2 & & & & & 0.5 & & & \\
\hline $9 \mathrm{H}, \mathrm{CC}$ & 85.70 & 3.13 & & & & 2.0 & & 0.3 & & 1.3 & & & & & & & & & & & 5.8 & \\
\hline $10 X-2,55-57$ & 87.76 & 3.21 & & & & 0.4 & & & & & & & & & & & & & 0.2 & & 7.6 & 0.4 \\
\hline $10 \times-4,55-57$ & 90.76 & 3.31 & & & & 0.5 & & & 0.2 & & & & & & & & & & & & 3.6 & 0.2 \\
\hline $10 \mathrm{X}, \mathrm{CC}$ & 95.30 & 3.47 & & & & 0.5 & & 1.4 & & 0.5 & & & & & & & & & 0.2 & & 5.3 & \\
\hline $11 X-2,55-57$ & 97.36 & 3.54 & & & & 0.4 & & & & & & & 0.4 & & 0.7 & & & & & & 11.0 & 0.2 \\
\hline $11 X-4,55-57$ & 100.36 & 3.59 & & & 1.8 & 0.7 & & & & & & & & & 0.4 & & & & & & 5.2 & 0.4 \\
\hline $11 \mathrm{X}, \mathrm{CC}$ & 104.90 & 3.64 & & & 0.2 & & & 0.7 & & 0.2 & & & & 1.6 & & & & & 0.2 & & 3.1 & \\
\hline $12 X-2,55-57$ & 106.96 & 3.66 & & & 1.4 & 0.4 & & & & & & & & & 0.4 & & & & 0.2 & & 3.4 & \\
\hline $12 \times-4,30-32$ & 109.71 & 3.69 & & & 1.5 & 1.4 & 0.2 & & & & & & & & 0.6 & & & 3.3 & & & 1.5 & \\
\hline $12 \mathrm{X}, \mathrm{CC}$ & 114.50 & 3.75 & & & 5.6 & & & 1.3 & & 1.1 & & & & & 0.3 & & & & 0.5 & & 5.6 & \\
\hline $13 \mathrm{X}-2,55-57$ & 116.56 & 3.77 & & & 4.9 & 0.2 & & & & & & & & 0.9 & 1.4 & & & & & & 4.2 & 0.2 \\
\hline $13 X-4,55-57$ & 119.56 & 3.81 & & & 2.2 & 0.2 & & & & & & & & & 3.0 & & & & 0.2 & 0.4 & 1.8 & \\
\hline $13 \mathrm{X}, \mathrm{CC}$ & 124.20 & 3.86 & & & 3.9 & 0.2 & & 0.4 & & 0.4 & & & & 1.1 & 0.4 & & & 0.2 & 0.4 & 0.4 & 6.1 & \\
\hline $14 X-2,55-57$ & 126.26 & 3.89 & & & 5.7 & 0.2 & & & & & & & & 4.3 & 0.4 & & & & & 0.2 & 5.0 & \\
\hline $14 \times-4,55-57$ & 129.26 & 3.92 & & & 3.2 & 0.3 & & & & & & & & & 4.3 & & & & 0.3 & & 1.9 & \\
\hline $14 \mathrm{X}, \mathrm{CC}$ & 133.90 & 3.97 & & & 2.1 & 0.7 & & 0.7 & & 0.2 & & & & 3.5 & 0.5 & & & & & & 4.9 & \\
\hline $15 X-2,55-57$ & 135.96 & 4.00 & & & 0.3 & 0.3 & & 0.3 & & & & & & 5.1 & & & & & 0.3 & 0.3 & 3.8 & \\
\hline $15 X-4,55-57$ & 138.96 & 4.03 & & & & & & & & & & & & 0.3 & & & & & & & 4.0 & \\
\hline $15 \mathrm{X}, \mathrm{CC}$ & 143.50 & 4.08 & & & 2.0 & 0.2 & & 0.9 & 0.7 & 0.5 & & & 0.5 & 2.0 & 0.3 & & & & 0.3 & 0.2 & 7.7 & \\
\hline $16 X-2,55-57$ & 145.56 & 4.11 & & & 0.9 & 0.2 & & & & & & & & & 0.7 & & & & 0.5 & & 2.2 & 0.4 \\
\hline $16 \times-4,30-32$ & 148.31 & 4.14 & & & 0.6 & 0.6 & & & & & & & & 0.6 & & & & & 0.3 & & 6.8 & 0.6 \\
\hline $16 \times-4,55-57$ & 148.56 & 4.14 & & & 1.7 & 0.2 & 0.2 & & & & & & 0.2 & 1.1 & & & & & & 0.2 & 5.5 & 0.4 \\
\hline $16 \mathrm{X}, \mathrm{CC}$ & 153.10 & 4.19 & & & 0.2 & 0.8 & & 1.0 & & & & & & 0.4 & & & & & 0.2 & & 5.0 & \\
\hline $17 X-2,55-57$ & 155.16 & 4.22 & & & 0.3 & 0.3 & & & & & & & & & & & & & & & 4.0 & 0.3 \\
\hline $17 X-4,55-57$ & 158.16 & 4.25 & & & 0.3 & 0.9 & & & & & & & 0.3 & 0.9 & & 3.5 & & & 0.6 & & 3.2 & 0.3 \\
\hline $17 \mathrm{X}, \mathrm{CC}$ & 162.80 & 4.31 & & & 1.1 & 0.6 & & 0.6 & & 0.1 & & & & 0.4 & & 0.4 & & & 0.1 & 0.8 & 5.0 & \\
\hline $18 \mathrm{X}-2,55-57$ & 164.86 & 4.33 & & & 0.2 & 5.5 & & 0.7 & & & & & & & & 3.3 & & & 0.9 & & 8.1 & \\
\hline $18 X-4,55-57$ & 167.86 & 4.36 & & & 1.0 & 0.2 & & & & & & & & 0.2 & & 1.5 & & & & 0.2 & 6.7 & \\
\hline $18 \mathrm{X}, \mathrm{CC}$ & 172.50 & 4.42 & & & 1.7 & 0.5 & & & & & & & & & & 1.9 & & & & 1.4 & 4.3 & \\
\hline $19 \mathrm{X}-2,55-57$ & 174.56 & 4.44 & & & 0.7 & & & & & & & & & & & & & & & & 2.4 & \\
\hline
\end{tabular}

Globobulimina affinis (d'Orbigny) = Bulimina affinis d'Orbigny, 1839, Foraminifères, in de la Sagra, Historie Physique et Naturelle de l'Ile de Cuba, vol. 8, p. 105, pl. 2, figs. 25-26.

Globobulimina ovata (d'Orbigny) = Bulimina ovata d'Orbigny, 1846, Foram. Foss. Vienne, p. 185, pl. 11, figs. 13-14.

Globobulimina spinescens $($ Brady) $=$ Bulimina pyrula var. spinscens Brady, 1884, Challenger-Exped., Rept. Zool., vol. 9, p. 400, pl. 50, figs. 11-12.

Globocassidulina subglobosa $(\mathrm{Brady})=$ Cassidulina subglobosa Brady, 1884, Challenger-Exped., Rept., Zool., vol. 9, p. 430, pl. 54, fig. 17.

Gyroidina altiformis Stewart and Stewart = Gyroidina soldanii var. altiformis Stewart and Stewart, 1930, J. Paleontol., vol. 4, p. 67, pl. 9, figs. $2 \mathrm{a}-\mathrm{c}$.
Gyroidina neosoldanii Brotzen, 1936, Sver. Geol. Unders., Avh., ser. C, no. 396, p. 158.

Gyroidina sp. 1.

Gyroidina sp. 2.

Hanzawaia concentrica (Cushman) = Truncatulina concentrica Cushman, 1918, U.S. Geol. Surv., Bull., vol. 676, p. 64, pl. 21, figs. 3a-c.

Hoeglundina elegans (d'Orbigny) $=$ Rotalia (Turbinulina) elegans d'Orbigny, 1826, Ann. Sci. Nat., ser. 1, vol. 7, p. 276, mod. 54.

Hyalinea balthica (Schroeter) = Nautilus balthicus Schroeter, 1783, Einleitung in der Conchylienkenntniss anch Linné, vol. 1, p. 20, pl. 1, fig. 2.

Karreriella bradyi (Cushman) = Gaudryina bradyi Cushman, 1911, U.S Natl. Mus., Bull., vol. 71, p. 67, text-figs. 107a-c. 


\begin{tabular}{|c|c|c|c|c|c|c|c|c|c|c|c|c|c|c|c|c|c|c|c|c|c|c|}
\hline $\begin{array}{l}\text { Core, section, } \\
\text { interval }(\mathrm{cm})\end{array}$ & $\begin{array}{l}\text { Depth } \\
\text { (mbsf) }\end{array}$ & $\begin{array}{l}\text { Age } \\
\text { (Ma) }\end{array}$ & 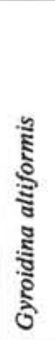 & 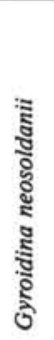 & 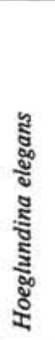 & 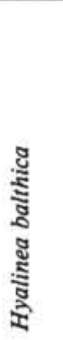 & 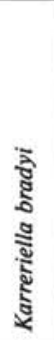 & 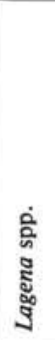 & 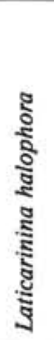 & 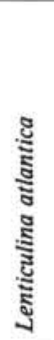 & 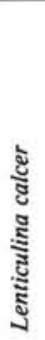 & 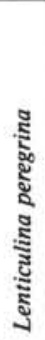 & 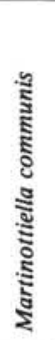 & 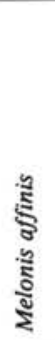 & 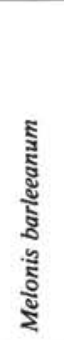 & 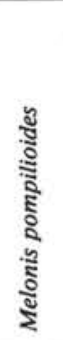 & 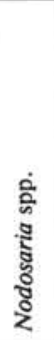 & 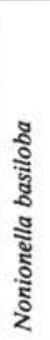 & 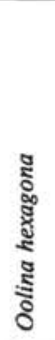 & $\begin{array}{l}\text { 客 } \\
\text { 응 } \\
0\end{array}$ & 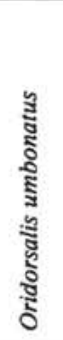 & 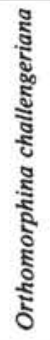 \\
\hline $19 X-4,55-57$ & 177.56 & 4.47 & & & 0.3 & & & & & & & & 0.3 & & & 10.7 & & & 0.6 & & 3.6 & 0.3 \\
\hline $19 X, C C$ & 182.20 & 4.53 & & & 2.8 & & & & & & & & & & & 4.2 & & & 0.2 & 0.9 & 3.1 & \\
\hline $20 \times-2,55-57$ & 184.26 & 4.55 & & & 5.2 & 0.2 & & & & & & & & & 0.2 & 7.5 & & & 0.4 & 0.8 & 4.6 & 0.4 \\
\hline $20 \times-4,55-57$ & 187.26 & 4.59 & & & 2.5 & 0.3 & & & & & & & 0.6 & & & 4.6 & & & & 0.9 & 5.3 & \\
\hline $20 \mathrm{X}, \mathrm{CC}$ & 191.80 & 4.64 & & & 0.3 & & & 0.8 & & & & & & & & 0.3 & & & & 0.3 & 7.4 & \\
\hline $21 \times-2,55-57$ & 193.86 & 4.66 & & & 0.4 & 0.2 & & 0.2 & & & & & & & & 0.6 & & & 0.9 & 0.2 & 9.6 & \\
\hline $21 \times-4,55-57$ & 196.86 & 4.70 & & & 2.9 & 0.3 & 0.3 & & & & & & 0.8 & & & 3.4 & & & 0.3 & 0.3 & 7.6 & \\
\hline $21 \mathrm{X}, \mathrm{CC}$ & 201.50 & 4.75 & & & 3.9 & & & & & & & & & & 0.4 & 3.2 & & & 0.4 & & 6.8 & \\
\hline $22 X-2,55-57$ & 203.56 & 4.77 & & & & & & & & & & & & & & 4.7 & & & & 0.2 & 7.2 & \\
\hline $22 X-4,55-57$ & 206.56 & 4.81 & & & 3.9 & 0.6 & & & & & & & 0.6 & & & 1.3 & & & 0.4 & 0.4 & 6.7 & 0.2 \\
\hline $22 \mathrm{X}, \mathrm{CC}$ & 211.10 & 4.86 & & & 1.0 & & & 0.2 & & & & & & & & 14.9 & & & & & 6.3 & \\
\hline $23 X-2,55-57$ & 213.16 & 4.88 & & & 0.6 & 0.3 & & & & & & & & & 0.3 & 2.5 & & & 0.6 & 0.8 & 7.9 & \\
\hline $23 X-4,55-57$ & 216.16 & 4.92 & & & 1.1 & 0.1 & 0.3 & 0.1 & & & & & 0.8 & & & 9.6 & & & & 0.3 & 2.8 & \\
\hline $23 \mathrm{X}, \mathrm{CC}$ & 220.80 & 4.97 & & & 1.8 & & & & & & & & & & & 2.5 & & & & & 7.2 & \\
\hline $24 X-2,55-57$ & 222.86 & 5.00 & & & 2.2 & & & & & & & & 0.3 & & & 1.4 & & & & 0.6 & 1.9 & \\
\hline $24 \mathrm{X}, \mathrm{CC}$ & 230.50 & 5.08 & & & & & & & & & & & 0.4 & 0.4 & & 7.5 & & & & & 5.0 & \\
\hline $25 X-2,55-57$ & 232.56 & 5.11 & & & & & & 0.3 & & & & & & & & 1.3 & & & 0.3 & & 11.0 & \\
\hline $25 X-4,55-57$ & 235.56 & 5.14 & & & 0.7 & 0.5 & & 0.2 & & & & & & & & 1.5 & & & & 0.2 & 10.4 & \\
\hline $25 \mathrm{X}, \mathrm{CC}$ & 240.10 & 5.19 & & & 3.0 & & & & & & & & & & & 2.4 & & & 0.3 & & 5.6 & \\
\hline $26 X-2,55-57$ & 242.16 & 5.22 & & & 3.9 & & & & & & & & 0.7 & & 0.4 & 7.1 & & & 0.4 & & 3.5 & \\
\hline $26 \mathrm{X}-4,55-57$ & 245.16 & 5.25 & & & 3.1 & & & & & & & & & & & 4.8 & & & & 0.3 & 10.4 & \\
\hline $26 \mathrm{X}, \mathrm{CC}$ & 249.80 & 5.31 & & & 0.8 & & & 0.4 & & & & & 0.2 & & & 10.2 & & & & & 4.6 & \\
\hline $27 X-2,55-57$ & 251.86 & 5.33 & & & 1.3 & & & & & & & & & & & 2.1 & & & & 0.3 & 9.4 & \\
\hline $27 X-4,55-57$ & 254.86 & 5.36 & & & 0.6 & & & & & & & & 1.4 & & & 8.0 & & & 0.3 & & 3.4 & \\
\hline $27 \mathrm{X}, \mathrm{CC}$ & 259.50 & 5.42 & & & 3.3 & & & 0.3 & & & & & 0.8 & & 0.5 & 2.5 & & & 0.3 & & 5.6 & \\
\hline $28 X-2,55-57$ & 261.56 & 5.44 & & & 1.7 & 0.2 & & & & & & & & & & 0.2 & & & & & 29.4 & 0.2 \\
\hline $28 X-4,55-57$ & 264.56 & 5.47 & & & & & & & & & & & & & & 3.4 & & & 0.3 & & 12.5 & \\
\hline $28 \mathrm{X}, \mathrm{CC}$ & 269.10 & 5.53 & & & 0.6 & & & & & & & & & 0.3 & 0.3 & 0.6 & & & & & 5.2 & \\
\hline $29 X-2,55-57$ & 271.16 & 5.55 & & & 1.2 & & & & & & & & 0.7 & & 0.2 & 0.5 & & & 0.2 & & 8.1 & \\
\hline $29 X, C C$ & 278.80 & 5.64 & & & 0.8 & & & & & & & & 1.6 & 7.4 & 1.2 & 5.9 & & & & & 6.3 & \\
\hline $30 \times-2,55-57$ & 280.86 & 5.66 & & & 1.7 & & & & & & & & & & & 1.0 & & & & & 4.7 & \\
\hline $30 \times-4,55-57$ & 283.86 & 5.70 & & & 0.7 & 0.3 & & & & & & & 1.0 & & 12.7 & 0.3 & & & 0.3 & & 2.0 & \\
\hline $30 \mathrm{X}, \mathrm{CC}$ & 288.50 & 5.87 & & & 0.6 & & & & & & & & 0.8 & 3.9 & 3.1 & 4.1 & & & & & 3.7 & \\
\hline $31 X-2,55-57$ & 290.56 & 5.95 & & & 2.3 & & & & 0.4 & & & & & & 13.6 & 3.0 & & & & & 5.7 & \\
\hline $31 X-4,55-57$ & 293.56 & 6.06 & & & 0.3 & & & & & & & & & & 16.7 & 1.4 & & & 0.3 & & 8.1 & \\
\hline $31 X, C C$ & 298.10 & 6.24 & & & 1.5 & & & & 1.0 & 0.2 & & & & 0.4 & 3.1 & 1.7 & & & & & 2.1 & \\
\hline $32 X-2,55-57$ & 300.16 & 6.32 & & & & & & & 0.4 & & & & 0.9 & & 17.1 & 1.3 & & & & & 3.4 & \\
\hline $32 \times-4,55-57$ & 303.16 & 6.43 & & & & & & & & & & & & & 8.0 & 4.0 & & & & & 3.4 & \\
\hline $32 \mathrm{X}, \mathrm{CC}$ & 307.80 & 6.61 & & & 0.3 & & & & & & & & 0.3 & 2.7 & 5.9 & 0.3 & & & & & 4.8 & \\
\hline $33 X-1,55-57$ & 308.36 & 6.63 & & & & & 0.2 & & & & & & 1.2 & & 3.3 & 0.7 & & & 0.2 & & 4.9 & \\
\hline $33 \times-4,55-57$ & 312.86 & 6.81 & & & & & & & & & & & & 1.2 & 15.7 & 0.3 & & & 0.6 & & 12.3 & \\
\hline $33 \mathrm{X}, \mathrm{CC}$ & 317.50 & 6.99 & & & & & & 0.4 & & & & & 0.7 & 8.6 & 5.4 & 0.4 & & & 0.4 & & 5.4 & \\
\hline $34 X-2,55-57$ & 319.56 & 7.07 & & & & & & & 0.6 & & & & 2.1 & & 8.1 & 0.3 & & & & & 5.4 & \\
\hline $34 X-4,55-57$ & 322.56 & 7.18 & & & 0.3 & & & & & & & & 0.3 & & 6.9 & 0.5 & & & 0.3 & & 5.7 & \\
\hline $34 \mathrm{X}, \mathrm{CC}$ & 327.20 & 7.36 & 1.0 & & 5.2 & & & & & 1.0 & & & & & 19.8 & 3.1 & & & & & 3.1 & \\
\hline $35 X-2,55-57$ & 329.26 & 7.44 & & & & & & & 2.2 & & & & & & 6.1 & 1.1 & 0.4 & & & 2.2 & 11.9 & \\
\hline $35 X-4,55-57$ & 332.26 & 7.55 & & 2.1 & 1.8 & & & & & 0.4 & & & 0.4 & 0.4 & 2.1 & 42.5 & & & & & 1.4 & \\
\hline $35 \mathrm{X}, \mathrm{CC}$ & 336.80 & 7.73 & & 2.2 & 2.5 & & & 0.2 & 0.4 & & & & 0.4 & 0.2 & 0.2 & 7.0 & & & & & 4.9 & \\
\hline $36 X-2,55-57$ & 338.86 & 7.81 & & & 1.9 & & & & & & & & & & & 40.0 & & & & & 7.8 & \\
\hline $36 \times-4,55-57$ & 341.86 & 7.92 & & 4.5 & & & & & & & & & & & & 48.4 & 0.4 & & & & 6.3 & \\
\hline
\end{tabular}

Lagena spp.

Laticarinina halophora $($ Stache) $=$ Robulina halophora Stache, 1864, Novara-Exped., Geol. Teil, vol. 1, p. 248,250 , pl. 23, figs. 28-29.

Lenticulina atlantica (Barker) $=$ Robulus atlanticus Barker, 1960, Soc. Econom. Paleontol. Mineral., Spec. Publ., vol. 9, p. 144, pl. 69, figs. 10-12.

Lenticulina calcer $($ Linné $)=$ Nautilus calcer Linné, 1727, Systema Naturae, 12th ed., p. 1162 , no. 272.

Lenticulina iota (Cushman) $=$ Cristellaria iota Cushman, 1923, U.S. Natl. Mus., Bull., vol. 104, p. 111, pl. 29, fig. 2; pl. 30, fig. 1.

Lenticulina peregrina $($ Schwager) $=$ Cristellaria peregrina Schwager, 1866, Novara-Exped., Geol. Teil., vol. 2, p. 245, pl. 7, fig. 89.
Lernella seranensis $($ Germeraad $)=$ Cassidulinoides seranensis Germeraad, 1946, in Geology, petrographical and paleontological results of explorations carried out from September, 1917 till June, 1919 in the Island of Ceram, ser. 3 (Geol.), no. 2, p. 72, pl. 5, figs. 5-6.

Martinottiella communis (d'Orbigny) = Clavulina communis d'Orbigny, 1826, Ann. Sci. Nat., ser. 1, vol. 7, p. 268, mod. 4.

Melonis affinis (Reuss) = Nonionina affinis Reuss, 1851, Deut. Geol. Ges., Z., vol. 3, p. 72, pl. 5, fig. 32 .

Melonis barleeanum $($ Williamson) $=$ Nonionina barleeana Williamson, 1858, Ray Soc., London, p. 32, pl. 3, figs. 68-69.

Melonis pompilioides (Fichtel and Moll) $=$ Nautilus pompilioides Fichtel and Moll, 1798, Testacea Microsc., p. 31, pl. 2, figs. a-c. 
Table 3 (continued).

\begin{tabular}{|c|c|c|c|c|c|c|c|c|c|c|c|c|c|c|c|c|c|c|c|c|c|c|c|}
\hline $\begin{array}{l}\text { Core, section, } \\
\text { interval }(\mathrm{cm})\end{array}$ & $\begin{array}{l}\text { Depth } \\
\text { (mbsf) }\end{array}$ & $\begin{array}{l}\text { Age } \\
\text { (Ma) }\end{array}$ & 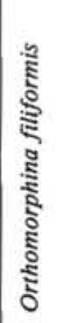 & 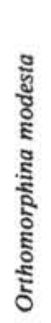 & 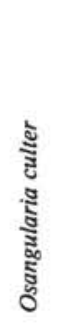 & 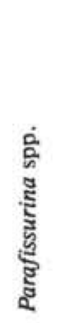 & 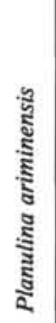 & 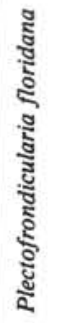 & 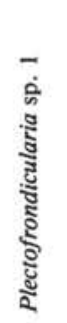 & 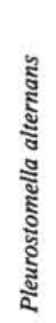 & 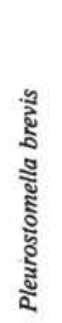 & 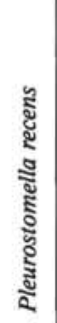 & 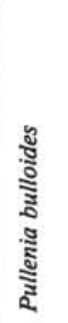 & 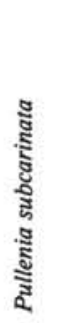 & 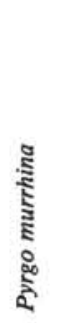 & 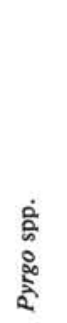 & 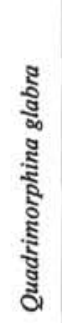 & 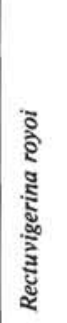 & 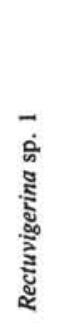 & 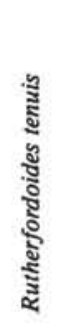 & 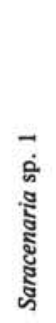 & 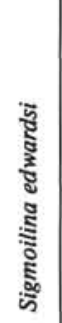 & 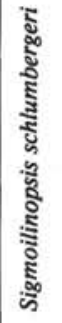 \\
\hline $1 \mathrm{H}-2,55-57$ & 2.06 & 0.05 & & & & & & & & & & & 0.9 & 0.9 & 0.9 & & 2.1 & & & & & 0.6 & 4.0 \\
\hline $1 \mathrm{H}-4,55-57$ & 5.06 & 0.12 & & & 0.3 & & & & & & & & 2.4 & 3.5 & 3.2 & & 0.5 & & & & & & 2.4 \\
\hline $1 \mathrm{H}, \mathrm{CC}$ & 9.60 & 0.23 & & & & 0.3 & & & & & & & 0.9 & 3.2 & 0.9 & 0.9 & 0.3 & & & 0.9 & & 0.9 & 4.7 \\
\hline $2 \mathrm{H}-2,55-57$ & 11.66 & 0.28 & & & 0.3 & 0.5 & 1.0 & & & & & & 2.6 & 1.0 & 1.3 & 1.8 & & & & & & & 2.6 \\
\hline $2 \mathrm{H}-4,55-57$ & 14.66 & 0.35 & & & 0.8 & & & & & & & & 1.7 & 1.7 & & 0.3 & 0.3 & & & & & & 1.9 \\
\hline $2 \mathrm{H}, \mathrm{CC}$ & 19.10 & 0.45 & & & & 0.2 & & & & & & & 0.2 & 1.9 & 0.6 & & 0.5 & & & & & & 1.8 \\
\hline $3 \mathrm{H}, \mathrm{CC}$ & 28.50 & 0.67 & & & & 0.3 & & & & & & & 1.9 & 1.3 & 1.3 & & 1.6 & & & & & & 3.2 \\
\hline $4 \mathrm{H}-4,55-57$ & 33.56 & 0.79 & & & 0.3 & & & & & & & & 0.3 & 2.8 & & & 1.4 & & & & & & 1.7 \\
\hline $4 \mathrm{H}, \mathrm{CC}$ & 38.00 & 0.90 & & & 0.5 & & & & & & & & 1.0 & 3.2 & 0.2 & & 1.5 & & & & & & 3.2 \\
\hline $5 \mathrm{H}-2,55-57$ & 40.06 & 0.95 & & & 1.2 & & & & & & & & 3.8 & 2.3 & & 1.2 & 1.2 & & & & & & 1.9 \\
\hline $5 \mathrm{H}-4,55-57$ & 43.06 & 1.04 & & & 1.4 & & & & & & & & 0.4 & 2.8 & & 1.1 & 2.1 & & & & & & 3.6 \\
\hline $5 \mathrm{H}, \mathrm{CC}$ & 47.50 & 1.21 & & & 1.2 & 0.9 & & & & & & & 2.2 & 1.1 & & & 1.7 & & & & & & 0.0 \\
\hline $6 \mathrm{H}-2,55-57$ & 49.56 & 1.29 & & & 0.6 & & & & & & & & 0.4 & 0.6 & & & 0.4 & & & & & & 2.4 \\
\hline $6 \mathrm{H}, \mathrm{CC}$ & 57.00 & 1.58 & & & 0.5 & 0.8 & & & & & & & 0.5 & 0.8 & 0.5 & & 2.6 & & & & & & 0.8 \\
\hline $7 \mathrm{H}-3,30-32$ & 60.31 & 1.71 & & & 0.8 & & & & 1.0 & & & & 1.6 & 0.8 & & 0.3 & 1.6 & & & & & & 1.6 \\
\hline $7 \mathrm{H}-5,100-102$ & 64.01 & 1.85 & & & & & 0.8 & 0.4 & 0.4 & & & & 2.0 & 0.8 & & 1.6 & 1.6 & & & & & & 2.0 \\
\hline $7 \mathrm{H}, \mathrm{CC}$ & 66.50 & 1.98 & & & 0.4 & & & & & & & & 6.3 & 9.2 & 0.9 & 0.2 & 0.6 & & & & & & 6.1 \\
\hline $8 \mathrm{H}-4,55-57$ & 71.56 & 2.35 & & & & & 0.3 & & & & & & 0.5 & 0.8 & & & 0.5 & & & & & & 0.3 \\
\hline $8 \mathrm{H}-6,55-57$ & 74.56 & 2.56 & & & & & & 0.3 & & & & & 1.2 & & & 0.3 & 0.6 & & & & & & 0.3 \\
\hline $8 \mathrm{H}, \mathrm{CC}$ & 76.10 & 2.67 & & & & & & & & & & & 0.5 & 0.2 & 0.5 & & 4.4 & & & & & & 2.7 \\
\hline $9 \mathrm{H}, \mathrm{CC}$ & 85.70 & 3.13 & & & & 0.3 & 1.8 & & & & & & 0.5 & & & & 0.3 & & & & & & 2.0 \\
\hline $10 X-2,55-57$ & 87.76 & 3.21 & & & 0.2 & & 0.6 & 0.6 & & & & & & 0.2 & & & 0.2 & & & & & & 0.2 \\
\hline $10 \times-4,55-57$ & 90.76 & 3.31 & & & 1.2 & & 0.2 & 0.7 & & & & & 4.5 & 0.7 & & 0.5 & 1.2 & & & & 0.2 & & 1.2 \\
\hline $10 \mathrm{X}, \mathrm{CC}$ & 95.30 & 3.47 & & & & 0.2 & & & & & & & 7.2 & 3.7 & & & & & & & & & 1.4 \\
\hline $11 X-2,55-57$ & 97.36 & 3.54 & & & & & 0.7 & 0.0 & & & & & 1.8 & 3.1 & & 0.7 & 0.2 & & & & & & 2.2 \\
\hline $11 X-4,55-57$ & 100.36 & 3.59 & & & & & & 0.7 & & & & & 1.1 & 2.2 & & 0.4 & & & & & & & 2.2 \\
\hline $11 \mathrm{X}, \mathrm{CC}$ & 104.90 & 3.64 & & & 0.9 & 0.2 & & & & & 0.2 & & & 2.0 & & & 1.1 & & & & & & 2.5 \\
\hline $12 X-2,55-57$ & 106.96 & 3.66 & & & 0.4 & & 0.2 & 0.2 & & & & & 1.1 & 2.3 & & 0.9 & & & & & & & 0.9 \\
\hline $12 X-4,30-32$ & 109.71 & 3.69 & & & 2.1 & & & 0.2 & & & & & 0.5 & 1.1 & & 0.6 & 0.3 & & & & & & 1.8 \\
\hline $12 \mathrm{X}, \mathrm{CC}$ & 114.50 & 3.75 & & & & & 0.3 & & & & & & & 4.8 & & 1.3 & 2.1 & & & & & & 1.1 \\
\hline $13 X-2,55-57$ & 116.56 & 3.77 & & & & 0.2 & 0.2 & & & & & & 0.9 & 2.1 & & 1.6 & 0.5 & & & & & & 0.5 \\
\hline $13 \times-4,55-57$ & 119.56 & 3.81 & & & & & 0.2 & 0.4 & & & & & 3.8 & 1.2 & & 0.8 & 0.4 & & & & 0.2 & & 2.4 \\
\hline $13 \mathrm{X}, \mathrm{CC}$ & 124.20 & 3.86 & & & & 0.2 & & & & & & & 2.0 & 1.1 & & & & & & & & & 3.3 \\
\hline $14 X-2,55-57$ & 126.26 & 3.89 & & & 0.2 & & & & & & & & 1.3 & 1.1 & & 0.7 & & & & & & & 0.9 \\
\hline $14 X-4,55-57$ & 129.26 & 3.92 & & & 0.5 & & & & & & & & 9.9 & 0.8 & & 1.6 & & & & & & 0.3 & 2.1 \\
\hline $14 \mathrm{X}, \mathrm{CC}$ & 133.90 & 3.97 & & & 1.2 & 0.2 & 0.2 & & & & & & 4.2 & 3.0 & 0.5 & & 0.2 & & & & 0.2 & & 4.4 \\
\hline $15 X-2,55-57$ & 135.96 & 4.00 & & & & & & & 0.3 & & & & 3.6 & 1.3 & & 0.5 & & & & & & & 1.0 \\
\hline $15 X-4,55-57$ & 138.96 & 4.03 & & & & 0.3 & & & & & 0.3 & & 8.3 & 2.6 & & & & & & & 0.3 & & 0.9 \\
\hline $15 \mathrm{X}, \mathrm{CC}$ & 143.50 & 4.08 & & & 0.3 & 0.2 & & 0.5 & 0.5 & & & & 10.7 & 1.4 & & & 0.2 & & & & & & 4.1 \\
\hline $16 \times-2,55-57$ & 145.56 & 4.11 & & & & & 0.2 & & & & & & 2.9 & 2.0 & 0.2 & & & & & & & & 0.7 \\
\hline $16 \times-4,30-32$ & 148.31 & 4.14 & & & 0.3 & 0.3 & 0.3 & & & & 0.3 & & 4.0 & 2.3 & & 2.6 & 0.3 & & & & & & 5.4 \\
\hline $16 X-4,55-57$ & 148.56 & 4.14 & & & 0.4 & 0.2 & 0.2 & & 0.2 & & 0.6 & 0.4 & 3.4 & 0.4 & & 1.1 & 0.2 & & & & 0.2 & & 3.0 \\
\hline $16 \mathrm{X}, \mathrm{CC}$ & 153.10 & 4.19 & & & & & & 0.4 & & & & & 16.8 & 2.9 & 0.4 & & 0.2 & & & & & & 0.4 \\
\hline $17 X-2,55-57$ & 155.16 & 4.22 & & & & & 0.5 & & 1.3 & & & & 7.8 & 2.0 & 0.5 & & 0.3 & & & & 0.3 & & 0.3 \\
\hline $17 X-4,55-57$ & 158.16 & 4.25 & & & 0.6 & 0.3 & 0.3 & & 0.3 & & 0.3 & 0.3 & 9.3 & 2.3 & & 0.9 & 0.3 & & & & & & 3.8 \\
\hline $17 \mathrm{X}, \mathrm{CC}$ & 162.80 & 4.31 & & & 0.4 & & & 0.4 & & & & & 3.8 & 1.0 & 0.3 & & 0.4 & & & & & & 5.3 \\
\hline $18 X-2,55-57$ & 164.86 & 4.33 & & & & & & & 2.0 & & & & 8.6 & 1.1 & & 1.5 & 0.9 & & & & 0.2 & & 5.3 \\
\hline $18 \times-4,55-57$ & 167.86 & 4.36 & 1.0 & & & & & 0.5 & 0.7 & & & & 6.7 & 1.0 & & 1.0 & 0.2 & & & & 0.2 & & 1.7 \\
\hline $18 \mathrm{X}, \mathrm{CC}$ & 172.50 & 4.42 & & & & & & & & & & & 2.9 & 1.4 & 1.0 & 0.2 & & & & & & & 0.5 \\
\hline $19 X-2,55-57$ & 174.56 & 4.44 & & & 0.2 & & 0.2 & 0.4 & & & & & 2.8 & 5.4 & & 0.7 & 0.2 & & & & 0.2 & & 1.1 \\
\hline
\end{tabular}

Nodosaria spp.

Nonionella basiloba Cushman and McCulloch, 1940, Allan Hancock Pacific Exped., vol. 6, no. 3, p. 163, pl. 18, figs. 3a-b.

Nonionella sp. 1.

Nuttallides umbonifera (Cushman) $=$ Pulvinulina umbonifera Cushman, 1933, Cushman Lab. Foram. Res., Contr., vol. 9, p. 90, pl. 9, figs. $9 \mathrm{a}-\mathrm{c}$.

Oolina hexagona $($ Williamson) = Entosolenia squamosa var. hexagona Williamson, 1848, Ann. Mag. Nat. Hist., ser. 2, vol. 1, p. 20, pl. 2, fig. 23.

Oolina spp.

Ophthalmidium pusillum (Earland) = Spiroloculina pusilla Earland, 1934, Discovery Repts., vol. 10, p. 47, pl. 1, figs. 3-4.
Oridorsalis umbonatus (Reuss) = Rotalina umbonata Reuss, 1851, Deut. Geol. Ges., Z., vol. 3, p. 73, pl. 5, fig. 35.

Orthomorphina challengeriana $($ Thalmann $)=$ Nodosaria perversa Brady, 1884, Challenger-Exped., Rept. Zool., vol. 9, p 512, pl. 64, figs. 2527. = Nodogenerina challengeriana Thalmann, 1937, Eclogae Geol. Helv., vol. 30, p. 341 .

Orthomorphina filiformis (d'Orbigny) = Nodosaria filiformis d'Orbigny, 1826, Ann. Sci. Nat., ser. 1, vol. 7, p. 253, mod. 14.

Orthomorphina modesta (Bermúdez) = Ellipsonodosaria modesta Bermúdez, 1937, Soc. Cubana Hist. Nat., Mem., vol. 11, p. 238, pl. 20, fig. 3 .

Orthomorphina spp. 


\begin{tabular}{|c|c|c|c|c|c|c|c|c|c|c|c|c|c|c|c|c|c|c|c|c|c|c|c|}
\hline $\begin{array}{l}\text { Core, section, } \\
\text { interval }(\mathrm{cm})\end{array}$ & $\begin{array}{l}\text { Depth } \\
\text { (mbsf) }\end{array}$ & $\begin{array}{l}\text { Age } \\
(\mathrm{Ma})\end{array}$ & 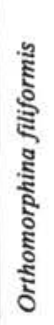 & 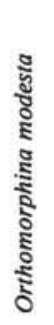 & 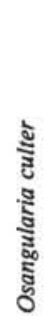 & 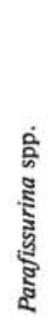 & 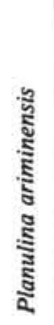 & 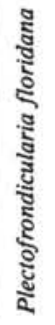 & 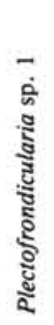 & 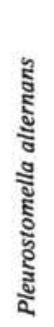 & 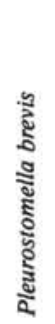 & 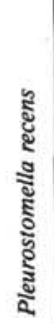 & 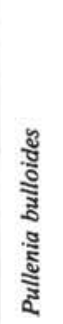 & 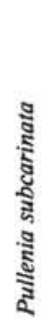 & 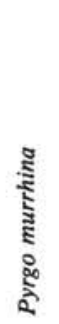 & $\begin{array}{l}\dot{0} \\
\text { के } \\
\dot{0} \\
\stackrel{0}{2} \\
\vdots\end{array}$ & 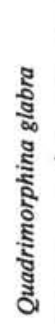 & 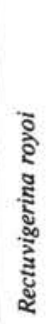 & 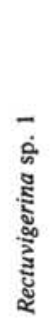 & 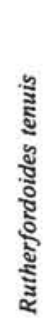 & 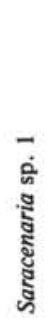 & 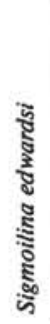 & 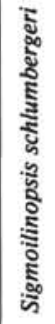 \\
\hline $19 \times-4,55-57$ & 177.56 & 4.47 & & & 1.8 & & & 0.6 & 0.3 & & 0.9 & 0.6 & 10.4 & 2.7 & & 0.6 & 0.9 & & & & 0.3 & & 0.3 \\
\hline $19 \mathrm{X}, \mathrm{CC}$ & 182.20 & 4.53 & & & 0.2 & 0.7 & & 1.3 & & & & & 2.2 & & 0.4 & & 0.7 & & & & & & 2.4 \\
\hline $20 \times-2,55-57$ & 184.26 & 4.55 & & & & & & 0.6 & & & 0.4 & & 7.9 & 1.7 & & 1.3 & 0.6 & & & & 0.2 & & 2.5 \\
\hline $20 \times-4,55-57$ & 187.26 & 4.59 & & & 0.3 & & & & & & & & 5.9 & 0.9 & & 1.2 & 0.3 & & & & & & 1.9 \\
\hline $20 \mathrm{X}, \mathrm{CC}$ & 191.80 & 4.64 & & & 0.3 & & & & & & & & 3.4 & 2.4 & & & 1.6 & & & & & & 5.3 \\
\hline $21 \times-2,55-57$ & 193.86 & 4.66 & & & & & & 0.2 & & & & & 2.6 & 2.6 & & & 0.6 & & & & & & 5.3 \\
\hline $21 \times-4,55-57$ & 196.86 & 4.70 & & & 0.3 & & & 0.8 & & & 0.3 & & 4.7 & 1.3 & & & & & & & 0.3 & & 5.0 \\
\hline $21 \mathrm{X}, \mathrm{CC}$ & 201.50 & 4.75 & & & & & & & & & & & 5.0 & 1.4 & 0.4 & & & & & & & & 0.4 \\
\hline $22 \times-2,55-57$ & 203.56 & 4.77 & & & & & & 0.7 & & & 0.2 & & 10.0 & 1.0 & & 1.5 & 0.5 & & & & & & 4.0 \\
\hline $22 \times-4,55-57$ & 206.56 & 4.81 & & & 0.2 & & & 0.4 & & & & & 16.4 & 1.3 & & & 0.4 & & & & & & 3.9 \\
\hline $22 \mathrm{X}, \mathrm{CC}$ & 211.10 & 4.86 & & & & & & 0.4 & & & & & 8.2 & 0.2 & 0.6 & & & & & & & & 4.5 \\
\hline $23 X-2,55-57$ & 213.16 & 4.88 & & & & & & 0.3 & & & & & 7.1 & 3.1 & & 0.3 & & & & & 0.3 & & 1.1 \\
\hline $23 \times-4,55-57$ & 216.16 & 4.92 & & & 0.6 & & & 0.1 & & & 0.3 & 0.1 & 5.3 & 0.8 & & 1.0 & & 0.1 & & & 0.1 & & 6.0 \\
\hline $23 \mathrm{X}, \mathrm{CC}$ & 220.80 & 4.97 & & & & & & & & & & & 2.2 & 4.0 & & & 0.7 & & & & & & 2.5 \\
\hline $24 X-2,55-57$ & 222.86 & 5.00 & & & & & 0.2 & 0.8 & & & & 0.2 & 15.4 & 3.5 & & 0.6 & & & & & 0.2 & & 6.3 \\
\hline $24 X, C C$ & 230.50 & 5.08 & & & & & & & & & 0.7 & & 0.0 & 1.1 & & & & & & & & & 3.6 \\
\hline $25 X-2,55-57$ & 232.56 & 5.11 & & & 0.5 & & 0.8 & & & & & & 3.0 & 2.7 & & & & & & & & & 9.6 \\
\hline $25 \times-4,55-57$ & 235.56 & 5.14 & & & 0.2 & & 0.2 & & & & & & 5.7 & 3.2 & & & 0.2 & & & & & & 6.0 \\
\hline $25 \mathrm{X}, \mathrm{CC}$ & 240.10 & 5.19 & & & 0.3 & & & 0.3 & & & & & 6.5 & 0.5 & & & 0.3 & & & & & & 5.9 \\
\hline $26 \times-2,55-57$ & 242.16 & 5.22 & & & 0.4 & & & 0.7 & & & 0.4 & & 3.5 & 2.5 & & & 0.4 & & & & & & 9.2 \\
\hline $26 \times-4,55-57$ & 245.16 & 5.25 & & & 1.4 & & & & & & 0.3 & & 4.8 & 3.9 & & 0.3 & 0.3 & & & & & & 4.2 \\
\hline $26 \mathrm{X}, \mathrm{CC}$ & 249.80 & 5.31 & & & & & & 1.9 & & & & & 6.0 & 1.3 & & 0.8 & 0.4 & & & & & & 5.0 \\
\hline $27 X-2,55-57$ & 251.86 & 5.33 & & & & & & 0.8 & & & & & 1.8 & 1.6 & & & 0.3 & & & & & & 3.4 \\
\hline $27 \times-4,55-57$ & 254.86 & 5.36 & & & 0.9 & & & 0.6 & & & 0.3 & & 5.2 & 2.3 & & 0.9 & & 0.6 & & & & & 7.2 \\
\hline $27 \mathrm{X}, \mathrm{CC}$ & 259.50 & 5.42 & & & 0.3 & 0.3 & & 0.8 & & & 0.3 & & 3.1 & 0.5 & & 0.5 & & & & & & & 6.4 \\
\hline $28 X-2,55-57$ & 261.56 & 5.44 & & & & & & & & & & & 1.0 & 1.2 & & & & & & & & & 3.4 \\
\hline $28 \times-4,55-57$ & 264.56 & 5.47 & & & & & & 0.9 & & & & & 0.6 & 0.9 & & & & & & & & & 3.8 \\
\hline $28 \mathrm{X}, \mathrm{CC}$ & 269.10 & 5.53 & & & & & & 0.3 & & & & & 0.6 & & & & & & & & & & 6.1 \\
\hline $29 \times-2,55-57$ & 271.16 & 5.55 & & & 1.2 & & & 1.8 & & & & & 0.9 & 0.5 & & 0.7 & & & & & 0.7 & & 5.5 \\
\hline $29 \mathrm{X}, \mathrm{CC}$ & 278.80 & 5.64 & & & & & & 0.8 & & & & & 0.8 & 0.4 & & & & & & & & & 4.3 \\
\hline $30 \times-2,55-57$ & 280.86 & 5.66 & & & 0.7 & & 0.3 & 1.7 & & & & & 0.7 & 0.7 & & & 2.4 & & & & & & 12.5 \\
\hline $30 \times-4,55-57$ & 283.86 & 5.70 & & & 0.3 & & & & & & & & 0.3 & 0.3 & & 1.0 & 0.3 & 0.3 & & & & & 8.1 \\
\hline $30 \mathrm{X}, \mathrm{CC}$ & 288.50 & 5.87 & & & 0.5 & & & 1.9 & & & & & 1.1 & 0.5 & 0.6 & & 0.2 & & & & & & 3.4 \\
\hline $31 X-2,55-57$ & 290.56 & 5.95 & & & 0.4 & & & 1.1 & & & & & 1.1 & 1.9 & & 1.5 & 2.3 & & & & & & 3.4 \\
\hline $31 \times-4,55-57$ & 293.56 & 6.06 & & & & & & & & & & & 1.4 & 0.0 & & & & & & & & & 2.2 \\
\hline $31 X, C C$ & 298.10 & 6.24 & & & 1.0 & 0.4 & & & & & & & 0.6 & 0.8 & 1.0 & & & & & & & & 10.3 \\
\hline $32 X-2,55-57$ & 300.16 & 6.32 & & & & & & 0.9 & & & 0.4 & & 3.0 & 0.9 & & 0.4 & & & & & & 0.4 & 11.1 \\
\hline $32 \times-4,55-57$ & 303.16 & 6.43 & & & & & & & & & & & 5.1 & 0.0 & & & 0.6 & & & & & & 4.5 \\
\hline $32 \mathrm{X}, \mathrm{CC}$ & 307.80 & 6.61 & & & & & & & & & & & 0.8 & 0.3 & & 0.8 & & & & & & & 4.8 \\
\hline $33 X-1,55-57$ & 308.36 & 6.63 & & & & & & & & & & & 1.6 & & & 0.7 & & & & & 0.2 & & 9.8 \\
\hline $33 \times-4,55-57$ & 312.86 & 6.81 & & & 0.6 & & & & & & & & 0.9 & 0.9 & & & & & & & & & 7.2 \\
\hline $33 \mathrm{X}, \mathrm{CC}$ & 317.50 & 6.99 & & & & & & & & & & & 1.1 & & & & & & & & & & 2.9 \\
\hline $34 X-2,55-57$ & 319.56 & 7.07 & & & & & & & & & 0.3 & & 3.3 & 0.6 & & 0.6 & & & & & & & 9.3 \\
\hline $34 X-4,55-57$ & 322.56 & 7.18 & & & 0.6 & & & 0.3 & & & & & 4.9 & 0.6 & & 0.6 & 0.6 & & & & & & 3.7 \\
\hline $34 \mathrm{X}, \mathrm{CC}$ & 327.20 & 7.36 & & & & & & & & & & & 4.2 & & & & 1.0 & & & & & & 6.3 \\
\hline $35 X-2,55-57$ & 329.26 & 7.44 & 0.4 & 1.4 & 0.7 & & & & & 0.7 & & & 2.9 & 0.4 & & 0.4 & 0.7 & & & & & & 4.7 \\
\hline $35 \times-4,55-57$ & 332.26 & 7.55 & & & 0.4 & & & & & & & & 2.5 & & & & & & 0.7 & & & & 1.4 \\
\hline $35 \mathrm{X}, \mathrm{CC}$ & 336.80 & 7.73 & & 0.2 & 1.1 & & & & & & 0.2 & & 3.1 & & 0.2 & 0.2 & & & & & & & 11.0 \\
\hline $36 \times-2,55-57$ & 338.86 & 7.81 & & & & & & & & & & & 0.4 & & & 0.7 & & & & & & & 7.0 \\
\hline $36 \times-4,55-57$ & 341.86 & 7.92 & 0.4 & & & & & & & & & & & & & & & & & & & & 0.4 \\
\hline
\end{tabular}

Osangularia culter (Parker and Jones) = Planorbulina culter var. farcata subvar. ungeriana Parker and Jones, 1865, Roy. Soc. London, Philos. Trans., vol. 155 , p. 421 , pl. 19, fig. 1.

Parafissurina spp.

Planulina ariminensis d'Orbigny, 1826, Ann. Sci. Nat., ser. 1, vol. 7, p. 280 , pl. 14, figs. 1-3.

Planulina exorna Phleger and Parker, 1951, Geol. Soc. Am., Mem., vol. 46 , pt. 2 , p. 32 , pl. 18 , figs. 5-7.

Plectofrondicularia floridana Cushman, 1930, Florida St. Geol. Surv., Bull., vol. 4, p. 41, pl. 8, fig. 1.

Plectofrondicularia sp. 1.

Pleurostomella alternans Schwager, 1866, Novara-Exped., Geol. Teil, vol. 2 , p. 238 , pl. 6 , fig. 79 .

Pleurostomella brevis Schwager, 1866, Novara-Exped., Geol. Teil, vol. 2 , p. 239 , pl. 6 , fig. 81 .
Pleurostomella recens Dervieux $=$ Pleurostomella rapa var. recens Dervieux, 1899, Soc. Geol. Italiana, Bol., vol. 18, p. 76, pl. 1, fig. 54.

Pullenia bulloides (d'Orbigny) $=$ Nonionina bulloides d'Orbigny, 1846 , Foram Foss. Vienne, p. 107, pl. 5, figs. 9-10.

Pullenia subcarinata (d'Orbigny) $=$ Nonionina subcarinata d'Orbigny, 1839, Voy. Am. Mérid., vol. 5, pt. 5, p. 28, pl. 5, figs. 23-24.

Pyrgo murrhina (Schwager) = Biloculina murrhina Schwager, 1866, Novara-Exped., Geol. Teil, vol. 2, p. 203, fig. 15.

Pyrgo spp.

Quadrimorphina glabra $($ Cushman $)=$ Valvulineria vilaredeboana var. glabra Cushman, 1927, Scripps Inst. Oceanogr., Bull., Technical Ser. vol. 1 , p. 161 , pl. 4 , figs. 5-6.

Rectuvigerina royoi Bermúdez and Fuenmayor, 1963, in Bermúdez and Seiglie, 1963, Inst. Ocean. Univ. Oriente, Bol., vol. 2, no. 2, p. 144, pl. 18 , fig. 9 . 
Table 3 (continued).

\begin{tabular}{|c|c|c|c|c|c|c|c|c|c|c|c|c|c|c|c|c|c|c|}
\hline $\begin{array}{l}\text { Core, section, } \\
\text { interval }(\mathrm{cm})\end{array}$ & $\begin{array}{l}\text { Depth } \\
\text { (mbsf) }\end{array}$ & $\begin{array}{l}\text { Age } \\
\text { (Ma) }\end{array}$ & 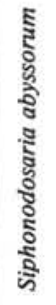 & 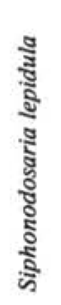 & 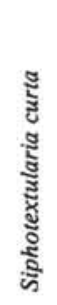 & 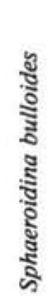 & 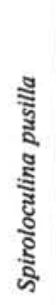 & 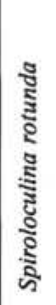 & 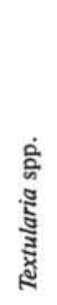 & 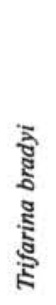 & 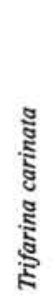 & 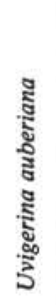 & 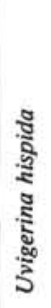 & 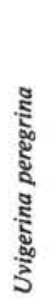 & 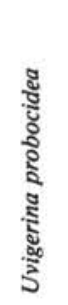 & 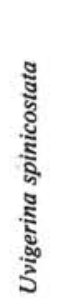 & 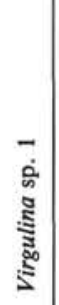 & 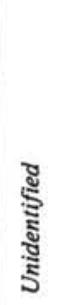 \\
\hline $1 \mathrm{H}-2,55-57$ & 2.06 & 0.05 & & & & 0.3 & 6.1 & 1.5 & 2.7 & & & 1.8 & & 0.6 & 2.4 & 0.6 & & 19.1 \\
\hline $1 \mathrm{H}-4,55-57$ & 5.06 & 0.12 & & & 1.1 & 0.3 & 4.3 & & 1.9 & & & 0.8 & & 0.3 & 3.0 & & 0.3 & 11.9 \\
\hline $1 \mathrm{H}, \mathrm{CC}$ & 9.60 & 0.23 & & & & 2.3 & 0.3 & & 7.6 & & 0.3 & 2.9 & 1.5 & 0.3 & 0.9 & & & 7.3 \\
\hline $2 \mathrm{H}-2,55-57$ & 11.66 & 0.28 & & & 0.5 & 1.8 & 0.5 & 2.6 & 3.1 & & & 0.8 & & 0.3 & 1.3 & & & 19.9 \\
\hline $2 \mathrm{H}-4,55-57$ & 14.66 & 0.35 & & & 0.2 & 0.8 & 0.3 & & 2.2 & & & 1.3 & & 1.0 & 5.7 & 2.2 & & 17.9 \\
\hline $2 \mathrm{H}, \mathrm{CC}$ & 19.10 & 0.45 & & & & 0.3 & 1.6 & & 1.0 & & & 0.2 & 0.5 & 0.4 & & 0.5 & & 3.4 \\
\hline $3 \mathrm{H}, \mathrm{CC}$ & 28.50 & 0.67 & & & & & 1.1 & & & & & 0.5 & & 0.8 & & & & 17.7 \\
\hline $4 \mathrm{H}-4,55-57$ & 33.56 & 0.79 & & 0.3 & & & 1.7 & & & & & 0.8 & 0.3 & 0.8 & 0.4 & 0.6 & & 20.4 \\
\hline $4 \mathrm{H}, \mathrm{CC}$ & 38.00 & 0.90 & & 1.2 & 0.2 & & 1.7 & & & & 0.5 & 0.5 & & & 0.2 & & 0.7 & 7.2 \\
\hline $5 \mathrm{H}-2,55-57$ & 40.06 & 0.95 & & 6.5 & & 0.8 & 1.5 & & & & 0.4 & & & 1.2 & 1.5 & 1.2 & 1.9 & 18.5 \\
\hline $5 \mathrm{H}-4,55-57$ & 43.06 & 7.8 & & & & 0.7 & 0.0 & & & & & 0.4 & & 0.4 & 5.3 & 2.1 & 0.4 & 19.9 \\
\hline $5 \mathrm{H}, \mathrm{CC}$ & 47.50 & 1.21 & 1.2 & 9.4 & & 0.8 & 0.6 & & & & 0.3 & 3.5 & & 0.2 & & 0.2 & 0.8 & 10.2 \\
\hline $6 \mathrm{H}-2,55-57$ & 49.56 & 1.29 & & 5.3 & & 1.2 & 0.4 & & & & 0.4 & 0.2 & & 0.4 & 5.1 & 0.8 & 1.0 & 23.5 \\
\hline $6 \mathrm{H}, \mathrm{CC}$ & 57.00 & 1.58 & 2.1 & 1.3 & & 0.8 & 0.5 & & & & 0.3 & 1.3 & & & 6.3 & 0.5 & & 10.2 \\
\hline $7 \mathrm{H}-3,30-32$ & 60.31 & 1.71 & & 5.9 & & & 2.1 & 0.3 & & & 0.3 & 0.5 & & & 2.8 & & 4.4 & 20.4 \\
\hline $7 \mathrm{H}-5,100-102$ & 64.01 & 1.85 & & 4.0 & & 0.8 & 1.2 & & & & & 0.8 & & & 2.4 & & 0.4 & 19.0 \\
\hline $7 \mathrm{H}, \mathrm{CC}$ & 66.50 & 1.98 & 4.6 & 9.6 & & 0.7 & 1.1 & & & & 0.2 & 1.5 & & 0.6 & 2.2 & & 1.3 & 15.5 \\
\hline $8 \mathrm{H}-4,55-57$ & 71.56 & 2.35 & & 2.9 & & & & & & & & 0.8 & & & 1.3 & & 1.6 & 19.9 \\
\hline $8 \mathrm{H}-6,55-57$ & 74.56 & 2.56 & & 4.3 & & & & & & & & 0.6 & & 3.4 & 6.2 & 2.2 & 6.2 & 15.5 \\
\hline $8 \mathrm{H}, \mathrm{CC}$ & 76.10 & 2.67 & 1.7 & 15.6 & & 0.3 & 1.5 & & & & & 0.8 & & 0.7 & 6.4 & 0.2 & 4.2 & 14.9 \\
\hline $9 \mathrm{H}, \mathrm{CC}$ & 85.70 & 3.13 & 1.3 & 5.3 & & 1.3 & 0.8 & & & & & & & 1.0 & 11.5 & 1.0 & 0.3 & 24.3 \\
\hline $10 X-2,55-57$ & 87.76 & 3.21 & & 0.2 & & & 0.6 & 0.2 & & & 0.2 & 0.4 & & 2.3 & 7.6 & 3.8 & 0.2 & 19.6 \\
\hline $10 X-4,55-57$ & 90.76 & 3.31 & & 2.2 & & 0.7 & 2.9 & & & & & 0.5 & & & 11.7 & 0.7 & & 23.2 \\
\hline $10 \mathrm{X}, \mathrm{CC}$ & 95.30 & 3.47 & 0.2 & 4.7 & & 0.7 & 1.2 & & & & & 0.5 & 0.2 & 1.4 & 5.8 & 0.9 & & 13.3 \\
\hline $11 X-2,55-57$ & 97.36 & 3.54 & & 1.8 & & 0.4 & 1.3 & & & & & 1.1 & & 0.7 & 11.9 & 5.3 & & 24.9 \\
\hline $11 X-4,55-57$ & 100.36 & 3.59 & & 1.1 & & 1.5 & 1.8 & & & & & 1.1 & 0.4 & & 12.9 & 0.4 & & 35.1 \\
\hline $11 \mathrm{X}, \mathrm{CC}$ & 104.90 & 3.64 & 0.4 & 1.3 & & 1.1 & 1.6 & & & & & & & & 6.5 & & & 38.0 \\
\hline $12 X-2,55-57$ & 106.96 & 3.66 & & 6.3 & & 0.2 & 2.5 & & & & & 0.4 & & 0.2 & 8.6 & 3.4 & & 24.0 \\
\hline $12 X-4,30-32$ & 109.71 & 3.69 & & 5.1 & 0.2 & 2.0 & 1.2 & & & 0.2 & 0.2 & 2.0 & & & 4.7 & 1.5 & & 33.8 \\
\hline $12 \mathrm{X}, \mathrm{CC}$ & 114.50 & 3.75 & & 5.1 & & & 3.5 & & & & & 0.3 & & 1.6 & 12.6 & & & 18.8 \\
\hline $13 \mathrm{X}-2,55-57$ & 116.56 & 3.77 & & 2.8 & & 0.7 & 0.2 & & 0.2 & & & 1.6 & & 0.7 & 16.8 & & & 22.8 \\
\hline $13 X-4,55-57$ & 119.56 & 3.81 & & 1.6 & & 3.4 & & & & & 0.2 & 0.6 & 0.2 & 0.4 & 7.1 & 0.4 & & 24.1 \\
\hline $13 \mathrm{X}, \mathrm{CC}$ & 124.20 & 3.86 & 0.7 & 5.6 & & 2.0 & 0.2 & & & & & 0.2 & & 0.4 & 8.7 & 1.1 & & 32.5 \\
\hline $14 X-2,55-57$ & 126.26 & 3.89 & & 4.8 & & 2.2 & 0.2 & & & & & 1.3 & & 5.2 & 5.2 & 0.4 & & 20.7 \\
\hline $14 X-4,55-57$ & 129.26 & 3.92 & & 2.7 & & 2.1 & & & & & & 1.1 & 1.6 & & 2.1 & & 0.3 & 27.2 \\
\hline $14 \mathrm{X}, \mathrm{CC}$ & 133.90 & 3.97 & 0.7 & 3.0 & & 2.6 & & & & & & 0.9 & & 1.6 & 2.8 & 0.9 & & 33.5 \\
\hline $15 X-2,55-57$ & 135.96 & 4.00 & & 1.0 & & 0.3 & 0.8 & & 1.3 & & 0.3 & 0.5 & & 2.8 & 9,4 & & 0.3 & 21.1 \\
\hline $15 X-4,55-57$ & 138.96 & 4.03 & & 4.6 & & 1.4 & 0.6 & & & & & 6.3 & 0.3 & 2.9 & 10.1 & 1.7 & 0.3 & 32.2 \\
\hline $15 \mathrm{X}, \mathrm{CC}$ & 143.50 & 4.08 & 0.3 & 4.8 & & 1.5 & 0.2 & & 1.4 & & & 0.9 & & 0.2 & 4.6 & & & 32.1 \\
\hline $16 \mathrm{X}-2,55-57$ & 145.56 & 4.11 & & 2.2 & & 0.7 & 0.0 & & 0.0 & & & 2.2 & & 2.2 & 5.7 & 0.7 & & 36.6 \\
\hline $16 \mathrm{X}-4,30-32$ & 148.31 & 4.14 & 0.3 & 4.3 & & 3.7 & 0.3 & & 3.1 & & & 2.6 & 0.3 & 0.9 & 5.1 & & & 21.1 \\
\hline $16 \times-4,55-57$ & 148.56 & 4.14 & 0.6 & 3.8 & & 1.1 & & & & & & 3.4 & 0.2 & 0.6 & 6.8 & & 0.2 & 15.1 \\
\hline $16 \mathrm{X}, \mathrm{CC}$ & 153.10 & 4.19 & 1.0 & 6.8 & & 2.9 & 0.2 & & 0.2 & & & 3.5 & & 0.2 & 3.3 & 0.2 & 0.4 & 28.6 \\
\hline $17 X-2,55-57$ & 155.16 & 4.22 & 0.3 & 10.9 & & 2.3 & 1.0 & & 3.8 & & & 1.3 & & 2.6 & 5.3 & & & 17.2 \\
\hline $17 X-4,55-57$ & 158.16 & 4.25 & 0.6 & 2.3 & & 2.9 & & & 0.3 & & & 1.2 & 0.3 & 1.2 & 5.8 & & & 17.7 \\
\hline $17 \mathrm{X}, \mathrm{CC}$ & 162.80 & 4.31 & 1.0 & 7.4 & & 2.7 & 1.0 & & & & & 1.0 & & & 3.4 & 0.8 & & 42.0 \\
\hline $18 X-2,55-57$ & 164.86 & 4.33 & & 7.3 & & 5.5 & 0.2 & & & & 0.2 & 0.9 & & 0.9 & 3.7 & & & 19.6 \\
\hline $18 X-4,55-57$ & 167.86 & 4.36 & & 9.4 & & 3.5 & & & & & 0.2 & 1.2 & & 0.2 & 3.5 & & & 16.9 \\
\hline $18 \mathrm{X}, \mathrm{CC}$ & 172.50 & 4.42 & 0.7 & 3.8 & & 0.2 & & & & & & 1.9 & & 0.5 & 5.8 & 1.7 & & 24.7 \\
\hline $19 X-2,55-57$ & 174.56 & 4.44 & & 1.7 & & 2.6 & 2.6 & & & & & 1.5 & & & 5.9 & 2.8 & & 33.6 \\
\hline
\end{tabular}

Rectuvigerina sp. 1.

Rosalina sp. 1.

Rutherfordoides tenuis $(\mathrm{Phleger}$ and Parker) $=$ Cassidulinoides tenuis Phleger and Parker, 1951, Geol. Soc. Am., Mem., vol. 46, pt. 2, p. 27, pl. 14, figs. 14-17.

Saracenaria latifrons $($ Brady) $=$ Cristellaria latifrons Brady, 1884, Challenger-Exped., Rept. Zool., vol. 9, p. 544, pl. 68, fig. 19, fig. 11a-b.

Saracenaria sp. 1.

Sigmoilina edwardsi (Schlumberger) = Planispirina edwardsi Schlumberger, 1887, Soc. Zool. France, Bull., vol. 12, p. 113, pl. 7, figs. $15-18$, text-fig. 8.

Sigmoilinopsis schlumbergeri $($ Silvestri) $=$ Sigmoilina schlumbergeri Silvestri, 1904, Accad, Pont. Romana Nuovi Lincei, Mem., vol. 22, p. 267,269 , text-figs. $6-7$.
Siphonina pulchra Cushman, 1919, Carnegie Inst., Publ., vol. 291, p. 42, pl. 14, fig. 7.

Siphonodosaria abyssorum (Brady) = Nodosaria abyssorum Brady, 1884, Challenger-Exped., Rept. Zool., vol. 9, p. 504, pl. 63, figs. 8-9.

Siphonodosaria lepidula (Schwager) $=$ Nodosaria lepidula Schwager, 1866, Novara-Exped., Geol. Teil, vol. 2, p. 210, pl. 5, figs. 27-28.

Siphotextularia curta (Cushman) = Textularia flintii var. curta Cushman, 1922, U.S. Natl. Mus., Bull., vol. 104 (pt. 3), p. 14, pl. 2, figs. 2-3.

Sphaeroidina bulloides d'Orbigny, 1826, Ann. Sci. Nat., ser. 1, vol. 7, p. 267 , mod. 65 .

Spiroloculina communis Cushman and Todd, 1944, Cushman Lab. Foram. Res., Spec. Publ., vol. 11, p. 63. 


\begin{tabular}{|c|c|c|c|c|c|c|c|c|c|c|c|c|c|c|c|c|c|c|}
\hline $\begin{array}{l}\text { Core, section, } \\
\text { interval }(\mathrm{cm})\end{array}$ & $\begin{array}{l}\text { Depth } \\
\text { (mbsf) }\end{array}$ & $\begin{array}{l}\text { Age } \\
(\mathrm{Ma})\end{array}$ & 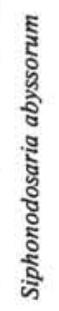 & 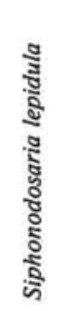 & 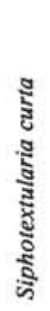 & 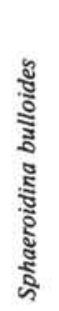 & 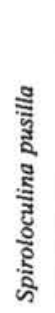 & 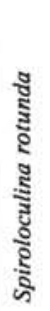 & 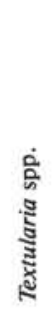 & 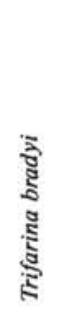 & 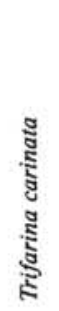 & 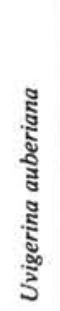 & 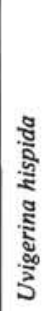 & 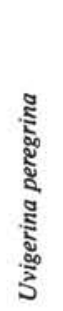 & 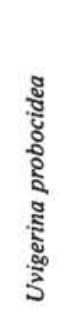 & 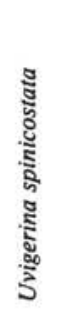 & 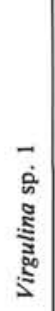 & 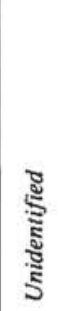 \\
\hline $19 \times-4,55-57$ & 177.56 & 4.47 & & 4.7 & & 2.1 & 0.3 & & & & & 4.5 & & 0.3 & 8.9 & 0.3 & & 20.5 \\
\hline $19 \mathrm{X}, \mathrm{CC}$ & 182.20 & 4.53 & & 7.0 & & 1.5 & 0.7 & & & & & 2.2 & 0.2 & 1.1 & 4.8 & 0.7 & & 26.0 \\
\hline $20 \times-2,55-57$ & 184.26 & 4.55 & & 5.4 & & 1.0 & 0.6 & & & & & 1.0 & & 0.8 & 6.3 & 1.0 & & 29.2 \\
\hline $20 \times-4,55-57$ & 187.26 & 4.59 & & 4.6 & & 0.3 & 0.3 & & & & & 2.5 & 0.9 & 0.9 & 7.4 & 2.8 & & 35.3 \\
\hline $20 \mathrm{X}, \mathrm{CC}$ & 191.80 & 4.64 & 0.3 & 8.2 & & 1.9 & 1.9 & & & & & 1.6 & & 0.8 & 3.2 & 1.6 & & 34.7 \\
\hline $21 X-2,55-57$ & 193.86 & 4.66 & & 2.1 & & 0.8 & 1.9 & & & & & 1.3 & & 1.1 & 5.3 & 3.4 & 0.2 & 38.1 \\
\hline $21 \times-4,55-57$ & 196.86 & 4.70 & & 3.9 & & 0.8 & 0.8 & & & & & 0.8 & 0.8 & 0.8 & 5.3 & 6.1 & 0.3 & 31.1 \\
\hline $21 \mathrm{X}, \mathrm{CC}$ & 201.50 & 4.75 & & 11.4 & & 2.1 & 2.5 & & & & & 3.9 & & 3.9 & 3.2 & & & 30.0 \\
\hline $22 X-2,55-57$ & 203.56 & 4.77 & & 4.2 & & 0.7 & & & & & & 1.5 & & 1.0 & 12.0 & 8.5 & & 22.7 \\
\hline $22 \times-4,55-57$ & 206.56 & 4.81 & & 2.6 & & 1.3 & 2.2 & & & & & 1.5 & 0.4 & 1.5 & 6.0 & 2.6 & & 23.1 \\
\hline $22 \mathrm{X}, \mathrm{CC}$ & 211.10 & 4.86 & 0.2 & 9.2 & & 0.6 & 0.2 & & & & & 1.4 & & 1.2 & 3.9 & 0.4 & & 26.4 \\
\hline $23 \mathrm{X}-2,55-57$ & 213.16 & 4.88 & & 6.8 & & 0.6 & & & & & & 1.4 & & 0.6 & 7.6 & 3.4 & & 26.6 \\
\hline $23 \times-4,55-57$ & 216.16 & 4.92 & & 4.9 & & 0.8 & 0.3 & & & & & 1.1 & 0.6 & 0.7 & 6.2 & 0.6 & & 35.1 \\
\hline $23 \mathrm{X}, \mathrm{CC}$ & 220.80 & 4.97 & & 4.0 & & 0.4 & 0.4 & & & & & 2.5 & & 4.0 & 2.2 & 0.4 & & 33.6 \\
\hline $24 X-2,55-57$ & 222.86 & 5.00 & & 2.7 & & 1.6 & 0.2 & & & & 0.5 & 0.8 & 0.2 & 3.0 & 1.9 & 5.3 & & 26.4 \\
\hline $24 \mathrm{X}, \mathrm{CC}$ & 230.50 & 5.08 & & 6.8 & & 1.4 & & & & & & 1.8 & & 1.1 & 6.8 & 3.9 & & 35.5 \\
\hline $25 X-2,55-57$ & 232.56 & 5.11 & & 4.7 & & 1.1 & 0.8 & & & & & 0.8 & & 1.4 & 10.4 & 3.8 & & 25.8 \\
\hline $25 X-4,55-57$ & 235.56 & 5.14 & & 1.5 & & 2.5 & 2.2 & & & & & 1.2 & 0.2 & 0.2 & 11.9 & 2.2 & & 29.3 \\
\hline $25 \mathrm{X}, \mathrm{CC}$ & 240.10 & 5.19 & & 7.8 & & 1.9 & 0.8 & & & & & 4.3 & 0.5 & 2.6 & 8.9 & 0.8 & & 33.6 \\
\hline $26 \mathrm{X}-2,55-57$ & 242.16 & 5.22 & & 3.2 & & 1.4 & 0.4 & & & & & 1.8 & & 0.7 & 4.2 & 1.1 & & 27.9 \\
\hline $26 \times-4,55-57$ & 245.16 & 5.25 & & 4.8 & & 1.4 & 0.6 & & & & & 2.3 & & 3.7 & 5.4 & 5.6 & & 24.8 \\
\hline $26 \mathrm{X}, \mathrm{CC}$ & 249.80 & 5.31 & & 6.5 & & 2.3 & 0.4 & & & & & 6.0 & 0.2 & 2.9 & 8.8 & 2.9 & & 21.9 \\
\hline $27 X-2,55-57$ & 251.86 & 5.33 & & 3.1 & & 0.5 & 0.8 & & & & & 3.1 & 0.3 & 3.7 & 9.4 & 6.8 & & 27.7 \\
\hline $27 X-4,55-57$ & 254.86 & 5.36 & & 4.6 & & 0.9 & 0.3 & & & & & 4.9 & & 0.9 & 9.5 & & & 23.0 \\
\hline $27 \mathrm{X}, \mathrm{CC}$ & 259.50 & 5.42 & & 8.4 & & 0.5 & 1.0 & & & & & 4.1 & & 1.0 & 5.9 & 2.8 & 0.3 & 34.3 \\
\hline $28 \times-2,55-57$ & 261.56 & 5.44 & & 3.4 & & 0.5 & 1.0 & & & & & 1.0 & 0.2 & 5.6 & 10.2 & 4.9 & & 19.2 \\
\hline $28 X-4,55-57$ & 264.56 & 5.47 & 0.6 & 7.8 & & 0.9 & 1.3 & & & & & 2.8 & & 0.9 & 9.4 & 7.2 & & 26.3 \\
\hline $28 \mathrm{X}, \mathrm{CC}$ & 269.10 & 5.53 & & 13.6 & & 3.9 & 1.9 & & & & & 5.5 & & 4.2 & 6.1 & 0.3 & & 34.0 \\
\hline $29 X-2,55-57$ & 271.16 & 5.55 & 0.5 & 7.6 & & 1.8 & 0.5 & & & & & 3.7 & & 5.1 & 9.9 & 0.7 & & 30.5 \\
\hline $29 \mathrm{X}, \mathrm{CC}$ & 278.80 & 5.64 & & 9.8 & & 3.9 & & & & & & 1.2 & & 3.1 & 2.3 & 1.6 & & 33.2 \\
\hline $30 \times-2,55-57$ & 280.86 & 5.66 & 0.3 & 4.7 & & & 4.7 & & & & & 1.4 & 0.3 & 2.4 & 4.7 & 1.7 & & 23.6 \\
\hline $30 \times-4,55-57$ & 283.86 & 5.70 & 0.3 & 6.2 & & 2.0 & 1.0 & & & & & 2.6 & 0.3 & 2.9 & 8.5 & 1.6 & & 20.5 \\
\hline $30 \mathrm{X}, \mathrm{CC}$ & 288.50 & 5.87 & & 8.3 & & 1.3 & 2.3 & & & & & 1.6 & & 6.2 & 5.7 & 2.9 & & 32.5 \\
\hline $31 \times-2,55-57$ & 290.56 & 5.95 & 0.4 & 6.1 & & 1.9 & 3.0 & & & & & 4.2 & & & 5.7 & 1.1 & & 20.8 \\
\hline $31 X-4,55-57$ & 293.56 & 6.06 & & 5.8 & & 3.3 & & & & & & 2.8 & & 8.9 & 11.9 & 9.4 & & 13.6 \\
\hline $31 X, C C$ & 298.10 & 6.24 & & 6.9 & & 1.9 & & & & & & 5.5 & & 0.4 & 8.6 & 8.2 & & 27.3 \\
\hline $32 X-2,55-57$ & 300.16 & 6.32 & 0.4 & 4.3 & & 1.7 & & & & & 0.4 & 5.6 & 0.4 & 7.7 & 9.8 & 3.4 & & 12.8 \\
\hline $32 \times-4,55-57$ & 303.16 & 6.43 & 0.6 & 4.5 & & 1.1 & & & & & 0.6 & 2.3 & 0.6 & 9.7 & 21.0 & 5.1 & & 13.6 \\
\hline $32 \mathrm{X}, \mathrm{CC}$ & 307.80 & 6.61 & & 13.4 & & 0.3 & 0.5 & & & & & 2.4 & 0.3 & 26.8 & 6.7 & 3.2 & & 16.1 \\
\hline $33 \mathrm{X}-1,55-57$ & 308.36 & 6.63 & 0.7 & 2.1 & & 2.3 & & & & & 0.2 & 2.3 & 0.5 & 22.2 & 7.5 & 1.9 & & 16.3 \\
\hline $33 X-4,55-57$ & 312.86 & 6.81 & & 9.3 & & 0.6 & & & & 0.6 & 0.6 & 1.8 & 0.3 & 2.4 & 3.0 & 1.8 & & 20.2 \\
\hline $33 \mathrm{X}, \mathrm{CC}$ & 317.50 & 6.99 & & 10.1 & & 1.1 & 0.4 & & & & & 4.7 & 0.4 & 7.6 & 11.5 & 6.5 & & 20.9 \\
\hline $34 X-2,55-57$ & 319.56 & 7.07 & & 3.3 & & 3.9 & & & & & & 1.5 & 0.3 & 9.3 & 6.0 & 3.9 & & 19.2 \\
\hline $34 X-4,55-57$ & 322.56 & 7.18 & 0.3 & 4.0 & & 5.1 & & & & & & 7.4 & 0.9 & & 2.0 & & & 22.6 \\
\hline $34 \mathrm{X}, \mathrm{CC}$ & 327.20 & 7.36 & 5.2 & 2.1 & & & & & 1.0 & & & 2.1 & & 11.5 & 3.1 & & & 9.4 \\
\hline $35 \times-2,55-57$ & 329.26 & 7.44 & 0.7 & 5.8 & & 0.7 & 0.4 & & 0.7 & & & 1.4 & & 0.4 & 2.5 & & & 11.2 \\
\hline $35 X-4,55-57$ & 332.26 & 7.55 & 0.4 & 2.5 & & 1.8 & & & 0.4 & & & 1.8 & & 8.8 & 6.0 & 1.4 & & 4.2 \\
\hline $35 \mathrm{X}, \mathrm{CC}$ & 336.80 & 7.73 & & 1.3 & & 1.1 & 0.2 & & 4.3 & & & 1.8 & 0.7 & 4.9 & 2.2 & 1.3 & & 22.9 \\
\hline $36 \times-2,55-57$ & 338.86 & 7.81 & 1.1 & 5.6 & & & & & & & & 0.4 & & 7.0 & 3.3 & 0.7 & & 5.2 \\
\hline $36 \times-4,55-57$ & 341.86 & 7.92 & 3.1 & 7.6 & & 0.4 & & & & & & 0.9 & & 4.0 & 1.8 & 1.8 & & 3.1 \\
\hline
\end{tabular}

Spiroloculina rotundata d'Orbigny, 1826, Ann. Sci. Nat., ser. 1, vol. 7 , p. 299.

Textularia spp.

Trifarina bradyi Cushman, 1923, U.S. Natl. Mus., Bull., vol. 104 (pt. 4), p. 99 , pl. 22 , figs. $3-9$.

Trifarina tricarinata d'Orbigny, 1826, Ann. Sci. Nat., ser. 1, vol. 7, p. 299, mod. 7.

Triloculina trigonula d'Orbigny, 1826, Ann. Sci. Nat., ser. 1, vol. 7, p. 299 , mod. 94.

Uvigerina auberiana d'Orbigny, 1839, Foraminifères, in de la Sagra, Historie Physique et Naturelle de l'Ile de Cuba, vol. 8, p. 106, pl. 2, figs. 23-24.
Uvigerina excellens Todd, in Cushman and McCulloch, 1948, Allan Hancock Pacific Exped., Repts., vol. 6, no. 5, p. 258, pl. 33, fig. 2. Uvigerina hispida Schwager, 1866, Novara-Exped., Geol. Teil, vol. 2, p. 249 , pl. 7 , fig. 95 .

Uvigerina peregrina Cushman, 1923, U.S. Natl. Mus., Bull., vol. 104 (pt. 4), p. 166, pl. 42, figs. 7-10.

Uvigerina probocidea Schwager, 1866, Novara-Exped., Geol. Teil, vol. 2, p. 250 , pl. 7, fig. 96 .

Uvigerina spinicostata Cushman and Jarvis, 1929, Cushman Lab. Foram. Res., Contr., vol. 5, no. 1, p. 12, pl. 3, figs. 9-10.

Virgulina sp. 1.

Virgulinella pertusa (Reuss) $=$ Virgulina pertusa Reuss, 1861, K. Akad. Wiss. Wien, Math-Naturwiss. Kl., Sitzungsber., vol. 42, p. 362. 
Table 4. Stratigraphic list of faunal event and paleomagnatic reversals for ODP Holes $725 \mathrm{C}, 726 \mathrm{~A}$, and 728A."

\begin{tabular}{|c|c|c|c|c|c|c|}
\hline & Event & Hole $725 \mathrm{C}$ & Hole $726 \mathrm{~A}$ & Hole 728A & Age (Ma) & $\begin{array}{c}\text { Source } \\
\text { of age }\end{array}$ \\
\hline FAD & Emiliania huxleyi & $25.78-28.50$ & $11.08-14.08$ & $10.78-13.78$ & 0.27 & 1,2 \\
\hline LAD & Pseudoemiliania lacunosa & $50.18-51.68$ & $25.08-26.98$ & $19.10-20.28$ & 0.46 & 1,2 \\
\hline B & Jaramillo & & $38.75-39.45$ & $40.70-42.20$ & 0.98 & 3 \\
\hline LAD & Calcidiscus macintyrei & & $61.28-63.60$ & $51.68-54.68$ & 1.45 & 4,5 \\
\hline LAD & Globigerinoides extremus ${ }^{*}$ & & & $57.00-66.50$ & 1.80 & $2,6,7$ \\
\hline \multirow[t]{2}{*}{ LAD } & Discoaster brouweri & & $63.60-64.71$ & $64.25-66.50$ & 1.89 & 4 \\
\hline & Matuyama/Gauss & & & $72.20-73.70$ & 2.47 & 8 \\
\hline $\mathrm{T}$ & Kaena & & & $78.80-80.30$ & 2.92 & 3 \\
\hline \multirow[t]{2}{*}{ B } & Mammoth & & & $84.80-86.90$ & 3.18 & 3 \\
\hline & Gauss/Gilbert & & & $92.90-94.40$ & 3.40 & 8 \\
\hline LAD & Sphenolithus abies & & $73.20-74.38$ & $95.30-96.48$ & 3.47 & 9,10 \\
\hline LAD & Reticulofenestra pseudoumbilica & & $77.12-77.85$ & $96.48-99.48$ & 3.56 & 7 \\
\hline FAD & Spongaster tetras tetras & & & $114.40-120.95$ & $3.83-3.85$ & 11 \\
\hline $\mathrm{T}$ & Cochiti & & & $121.70-125.35$ & 3.88 & 3 \\
\hline B & Cochiti & & & $131.40-132.89$ & 3.97 & 3 \\
\hline $\mathrm{T}$ & Nunivak & & & $142.60-144.70$ & 4.10 & 3 \\
\hline B & Nunivak & & & $152.08-155.80$ & 4.24 & 3 \\
\hline $\mathrm{T}$ & Sidufjall & & & $167.08-168.41$ & 4.40 & 3 \\
\hline B & Sidufjall & & & $176.75-178.22$ & 4.47 & 3 \\
\hline $\mathrm{T}$ & Thvera & & & $184.67-186.32$ & 4.57 & 3 \\
\hline FAD & Discoaster quinqueramus & & & $230.50-231.68$ & 4.98 & 12 \\
\hline FAD & Globorotalia tumida tumida & & $97.55-102.10$ & $240.10-249.80$ & 5.20 & 7,13 \\
\hline \multirow[t]{2}{*}{ LAD } & Stichocorys johnsoni** & & & $278.80-284.15$ & $5.70-5.80$ & 14 \\
\hline & Stichocorys delmontensis $\rightarrow S$. peregrina & & & $298.10-307.80$ & $6.10-6.70$ & 14 \\
\hline LAD & Calocycletta caepa & & & $303.45-307.80$ & $6.20-6.60$ & 14 \\
\hline LAD & Diartus hughesi & & & $322.85-327.20$ & $7.10-7.20$ & 14 \\
\hline LAD & Botryostrobus miralestensis & & & $342.15-346.40$ & $8.10-8.20$ & 14 \\
\hline
\end{tabular}

a Note: FAD, first appearance datum; LAD, last appearance datum; T, top; B, bottom; $\rightarrow$ evolutionary transition; * $=$ G. obliquus extremus in Berggren et al. (1985); ** $=E$. cf. diaphanes in Johnson and Nigrini (1985).

b References for age; 1, Thierstein et al. (1977); 2, Berggren et al. (1980); 3, Berggren et al. (1985); 4, Backman and Shackleton (1983); 5, Backman et al. (1983); 6, Thompson and Sciarrillo (1978); 7, Keigwin (1982); 8, Mankinen and Dalrymple (1979); 9, Monechi et al. (1985); 10, Rio (1982); 11, Johnson et al. (1989); 12, Backman (pers. comm., March 1989); 13, Saito et al. (1975); 14, Johnson and Nigrini (1985).

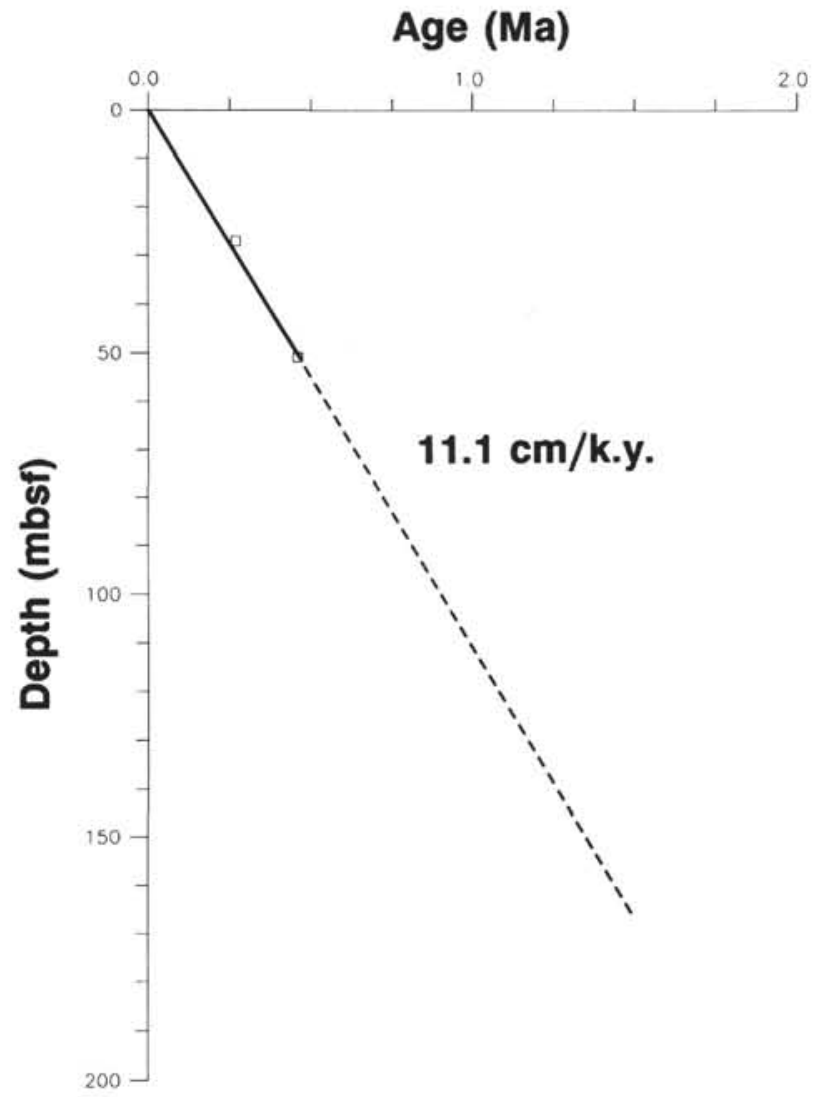

Figure 3. Age/depth relationships for biostratigraphic events in ODP Hole 725C. For a detailed listing of events, see Table 4.

\section{REFERENCES}

Backman, J., and Shackleton, N. J., 1983. Quantitative biochronology of Pliocene and early Pleistocene calcareous nannofossils from the Atlantic, Indian and Pacific oceans. Mar. Micropaleontol., 8:141170.

Backman, J., Shackleton, N. J., and Tauxe, L., 1983. Quantitative nannofossil correlation to open ocean deep-sea sections from Plio-Pleistocene boundary at Vrica, Italy. Nature, 304(5922):156-158.

Berggren, W. A., Burckle, L. H., Cita, M. B., Cooke, H. B. S., Funnell, B. M., Gartner, S., Hays, J. D., Kennett, J. P., Opdyke, N. D., Pastouret, L., Shackleton, N. J., and Takayanagi, Y., 1980. Towards a Quaternary time scale. Quat. Res., 13:277-302.

Berggren, W. A., Kent, D. V., and Van Couvering, J. A., 1985. The Neogene: Part 2. Neogene geochronology and chronostratigraphy. In Snelling, N. J. (Ed.), The Chronology of the Geological Record: Geol. Soc. Mem. (London), 10:211-260.

Boltovskoy, E., 1978. Late Cenozoic benthonic foraminifera of the Ninetyeast Ridge (Indian Ocean). Mar. Geol., 26:139-175.

Chang, Y., 1967. Accuracy of fossil percentage estimation. J. Paleontol., 41:500-502.

Corliss, B. H., 1979a. Recent deep-sea benthonic foraminiferal distributions in the southeast Indian Ocean: inferred bottom-water routes and ecological implications. Mar. Geol., 31:115-138. , 1979b. Taxonomy of Recent deep-sea benthonic foraminifera from the southeast Indian Ocean. Micropaleontology, 25:1-19.

Culver, S. J., and Buzas, M. A., 1980. Distribution of Recent benthic foraminifera off the North American Atlantic coast. Smithsonian Contr. Mar. Sci., 6:1-512.

1981. Distribution of Recent benthic foraminifera in the Gulf of Mexico: Volume II. Smithsonian Contr. Mar. Sci., 8:413-898.

, 1982. Distribution of Recent benthic foraminifera in the Caribbean Region. Smithsonian Contr. Mar. Sci., 14:1-382.

Currie, R. I., Fisher, A. E., and Hargreaves, P. M., 1973. Arabian Sea upwelling. In Zeitschel, B., and Gerlach, S. A., (Eds.), The Biology of the Indian Ocean, Ecological Studies, vol. 3: Berlin (SpringerVerlag), 37-52. 
Deuser, W. G., Ross, E. H., and Mlodzinska, Z. J., 1978. Evidence for and rate of denitrification in the Arabian Sea. Deep-Sea Res., 25: 431-445.

Hermelin, J.O.R., and Shimmield, G. B., 1990. The importance of the oxygen minimum zone and sediment geochemistry on the distribution of Recent benthic foraminifera from the NW Indian Ocean. Mar. Geol., 91:1-29.

Johnson, D. A., and Nigrini, C. A., 1985. Synchronous and time-transgressive radiolarian datum levels in the equatorial Indian and Pacific Oceans. Mar. Micropaleontol., 9:489-523.

Johnson, D. A., Schneider, D. A., Nigrini, C. A., and Caulet, J. P., 1989. Pliocene-Pleistocene radiolarian events and magnetostratigraphic calibrations for the tropical Indian Ocean. Mar. Micropaleontol., 14:33-66.

Keigwin, L. D., Jr., 1982. Neogene planktonic foraminifera from Deep Sea Drilling Project Sites 502 and 503. In Prell, W. L., Gardner, J. V. et al., Init. Repts. DSDP, 68: Washington (U.S. Govt. Printing Office), 269-288.

Krey, J, and Babenerd, B., 1976. Phytoplankton Production: Atlas of the International Indian Ocean Expedition. Intergovermental Oceanographic Commission, UNESCO.

Kuz'menko, L. O., 1974. Primary productivity of the northern Arabian Sea. Oceanology, 20:164-167.

Mankinen, E. A., and Dalrymple, G. B., 1979. Revised geomagnetic polarity time scale for the interval 0-5 m.y.B.P. J. Geophys. Res., 84: 615-26.

Monechi, S., Bleil, U., and Backman, J., 1985. Magnetobiochronology of late Cretaceous-Paleogene and late Cenozoic pelagic sedimentary sequences from the northwest Pacific (DSDP Leg 86, Site 577). In Heath, G. R., Burckle, L. H., et al., Init. Repts. DSDP, 86: Washington (U.S. Govt. Printing Office), 787-797.

Mountain, G. S., and Prell, W. L., 1989. Geophysical reconnaissance for ODP Leg 117 in the northwest Indian Ocean. in Prell, W. L., Niitsuma, N., et al., Proc. ODP, Init. Repts., 117: College Station, TX (Ocean Drilling Program), 51-64.

Naqvi, S.W.A., Noronha, R. J., and Gangadhara Reddy, C. V., 1982. Denitrification in the Arabian Sea. Deep-Sea Res., 29:459-469.

Pflum, C., and Frerichs, W., 1976. Gulf of Mexico deep-water foraminifers. Cushman Found. Foram. Res., Spec. Publ., 14:1-122.

Prell, W. L., and Curry, W. B., 1981. Faunal and isotopic indices of monsoonal upwelling: western Arabian Sea. Oceanol. Acta, 4:9198.

Prell, W. L., Niitsuma, N., et al., 1989. Proc. ODP, Init. Repts., 117: College Station, TX (Ocean Drilling Program).

Prell, W. L., and Streeter, H. F., 1982. Temporal and spatial patterns of monsoonal upwelling along Arabia: A modern analogue for the interpretation of Quaternary SST anomalies. J. Mar. Res., 40:143155.

Price, N. B., and Shimmield, G. B., 1987. Cruise report for R/V Charles Darwin, Leg 17, NW Indian Ocean. Unpubl. report, Univ. Edinburgh.

Quasim, S. Z., 1982. Oceanography of the northern Arabian Sea. DeepSea Res., 29:1041-1068.

Rio, D., 1982. The fossil distribution of coccolithophore genus Gephyrocapsa Kamptner and related Plio-Pleistocene chronostratigraphic problem. In Prell, W. L., Gardner, J. V. et al., Init. Repts. DSDP, 68: Washington (U.S. Govt. Printing Office), 325-343.

Saito, T., Burckle, L. H., and Hays, J. D., 1975. Late Miocene to Pleistocene biostratigraphy of equatorial Pacific sediments. In Saito, T., and Burckle, L. H. (Eds.), Late Neogene epoch boundaries. Micropaleontology, Spec. Pap. 1:226-244.

Slater, R. D., and Kroopnick, P., 1984. Controls on dissolved oxygen distribution and organic carbon deposition in the Arabian Sea. In Haq, B. U., and Milliman, J. D. (Eds.), Marine Geology and Ocean- ography of Arabian Sea and coastal Pakistan. New York (Van Nostrand Reinhold Company), 305-313.

Stein, C. A., and Cochran, J. R., 1985. The transition between the Sheba Ridge and Owen Basin: rifting of old oceanic lithosphere. Geophys. J. Roy. Astr. Soc., 81:47-74.

Thierstein, H. R., Geitzenauer, K. R., Molfino, B., and Shackleton, N. J., 1977. Global synchroneity of late Quaternary coccolith datum levels: validation by oxygen isotopes. Geology, 5:400-404.

Thompson, P. R., and Sciarrillo, J. R., 1978. Planktonic foraminiferal biostratigraphy in the Equatorial Pacific. Nature, 276:29-33.

Whitmarsh, R. B., 1979. The Owen Basin off the south-east margin of Arabia and the evolution of the Owen Fracture Zone. Geophys. J. Roy. Astr. Soc., 58:441-470.

Wyrtki, K., 1971. Oceanographic Atlas of the International Indian Ocean Expedition. Washington (National Science Foundation).

1973. Physical oceanography of the Indian Ocean. In Zeitschel, B., and Gerlach, S. A., (Eds.), The Biology of the Indian Ocean, Ecological Studies, vol 3: Berlin (Springer-Verlag), 18-36.

Date of initial receipt: 2 October 1989

Date of acceptance: 21 March 1990

Ms 117B-130

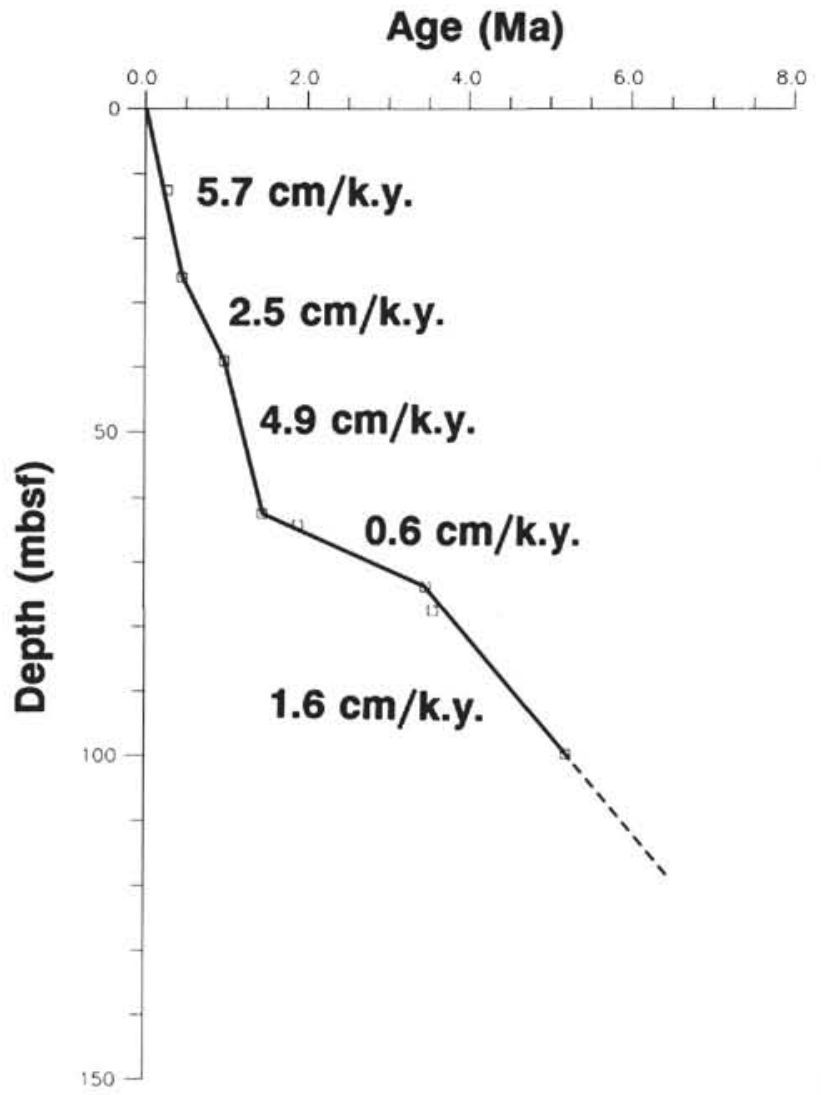

Figure 4. Age/depth relationships for biostratigraphic and magnetostratigraphic events in ODP Hole 726A. For a detailed listing of events, see Table 4. 


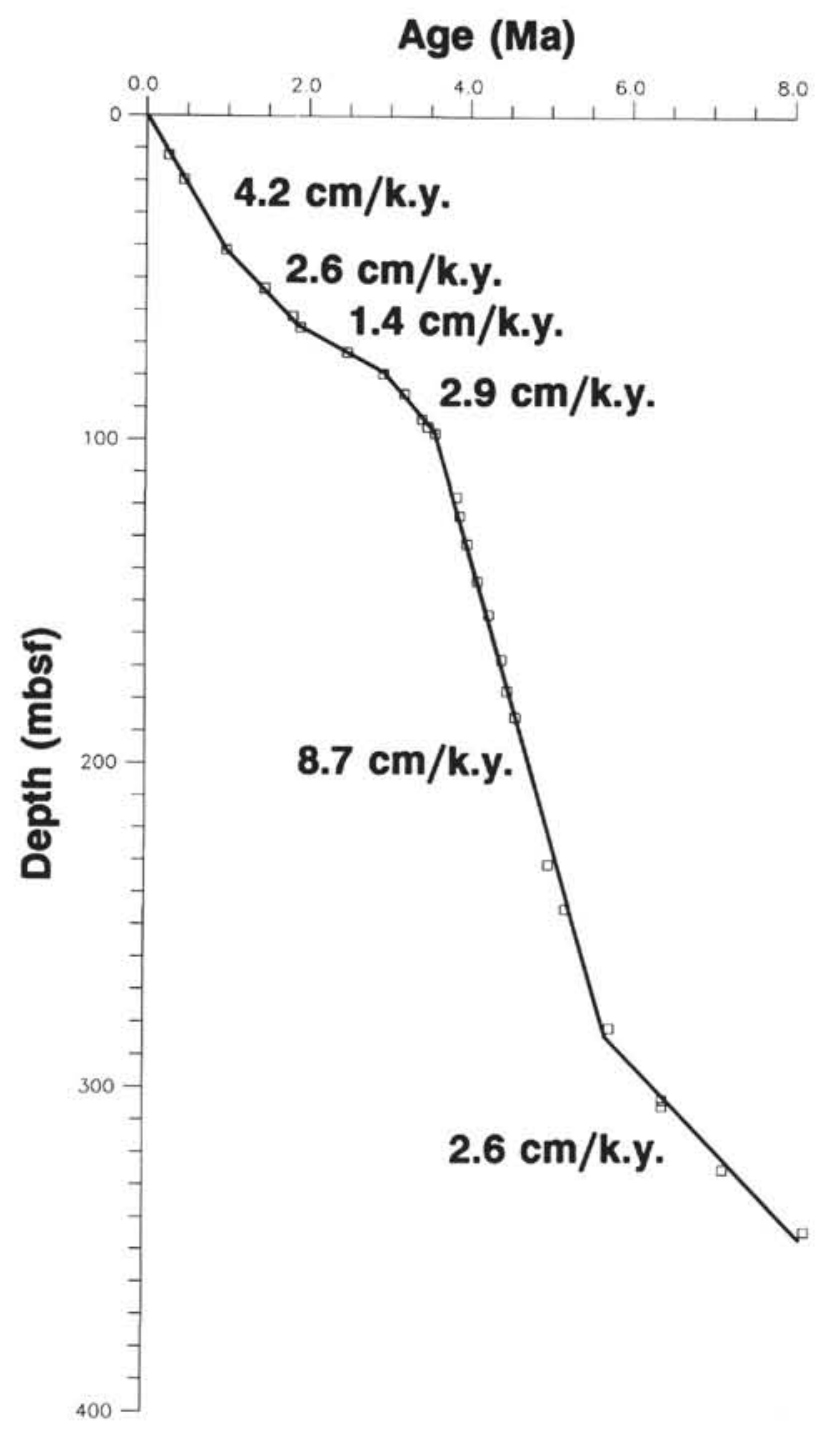

Figure 5. Age/depth relationships for biostratigraphic and magnetostratigraphic events in ODP Hole 728A. For a detailed listing of events, see Table 4.

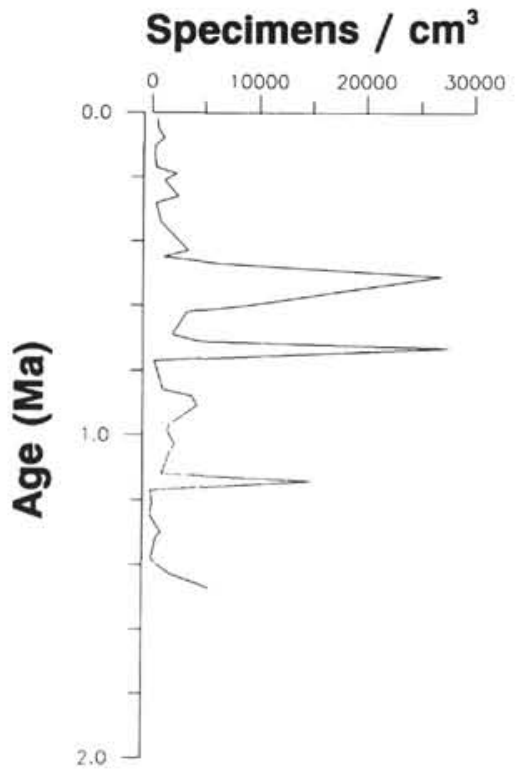

Figure 6. Calculated total number of benthic foraminifers per cubic centimeter of sediment plotted vs. time for ODP Hole $725 \mathrm{C}$. 

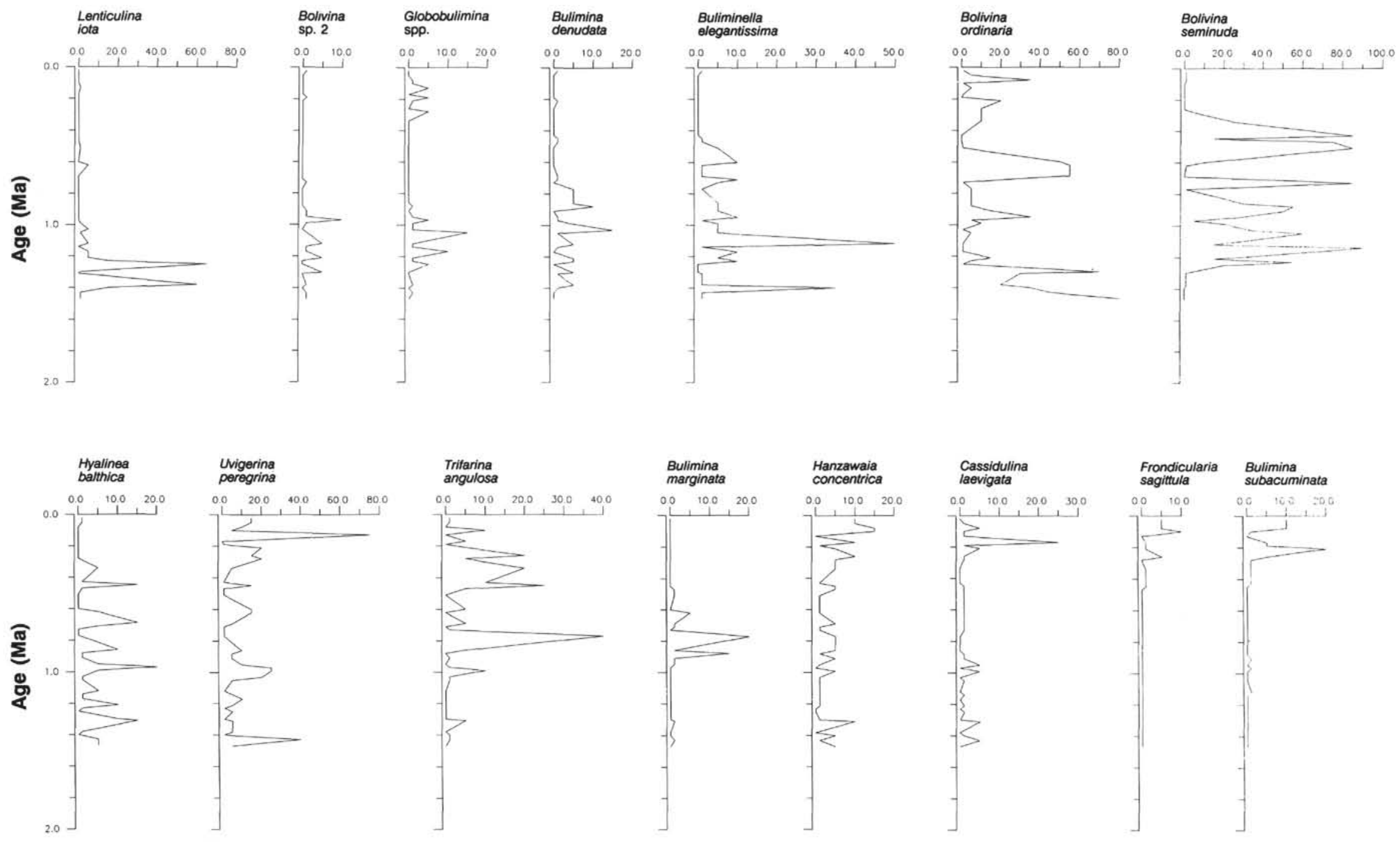

Figure 7. Relative abundance curves for selected benthic foraminiferal species from ODP Hole 725C. (Note the different percentage scale for Bolivina ordinaria, Bolivina seminuda, Lenticulina iota, and Uvigerina peregrina). 


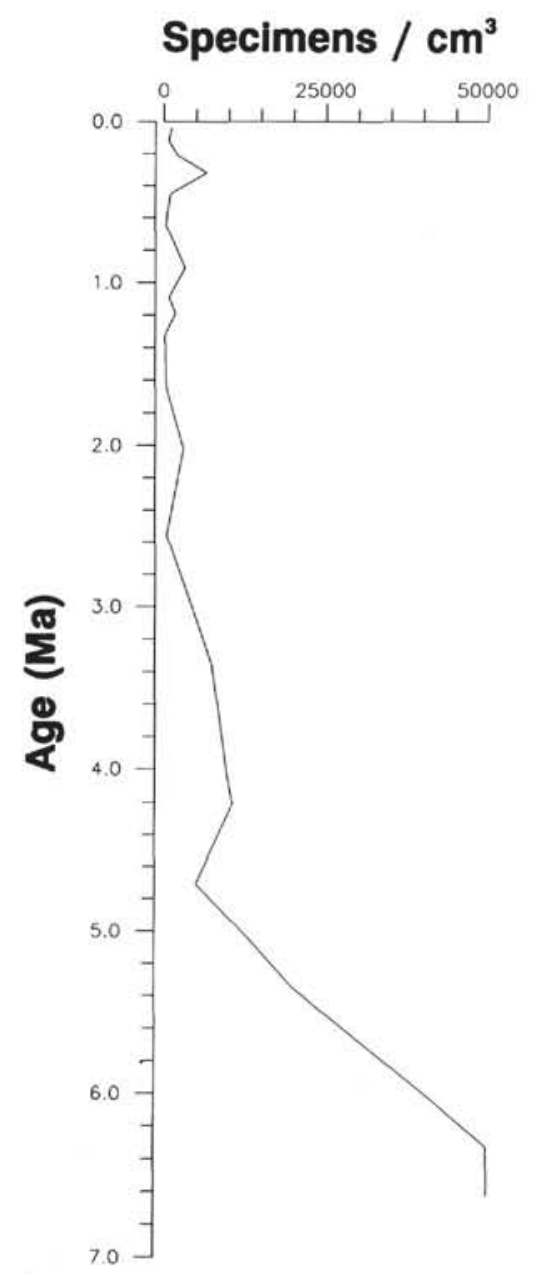

Figure 8. Calculated total number of benthic foraminifers per cubic centimeter of sediment plotted vs. time for ODP Hole 726A. 
BENTHIC FORAMINIFERAL FAUNAS, SITES 725, 726, 728
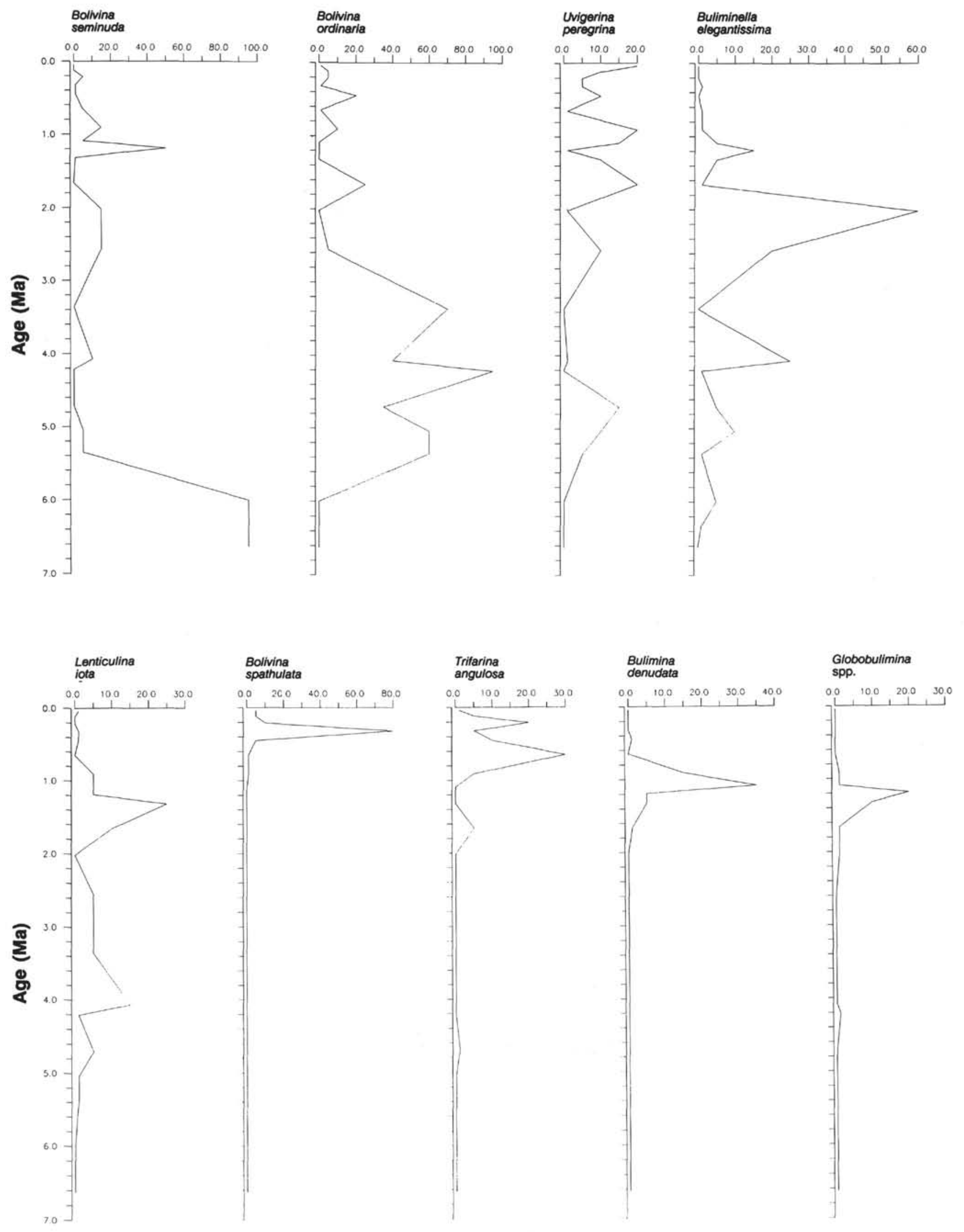

Figure 9. Relative abundance curves for selected benthic foraminiferal species from ODP Hole 726A. (Note the different percentage scale for Bolivina ordinaria, Bolivina seminuda, Bolivina spathulata, and Buliminella elegantissima). 


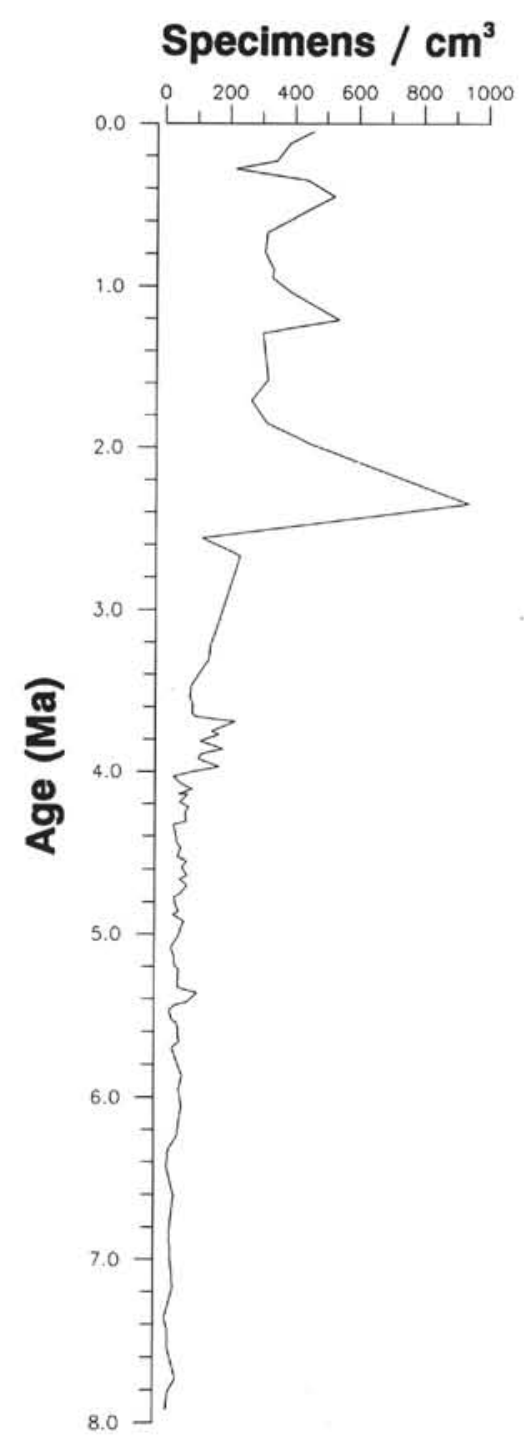

Figure 10. Calculated total number of benthic foraminifers per cubic centimeter of sediment plotted vs. time for ODP Hole 728A. 


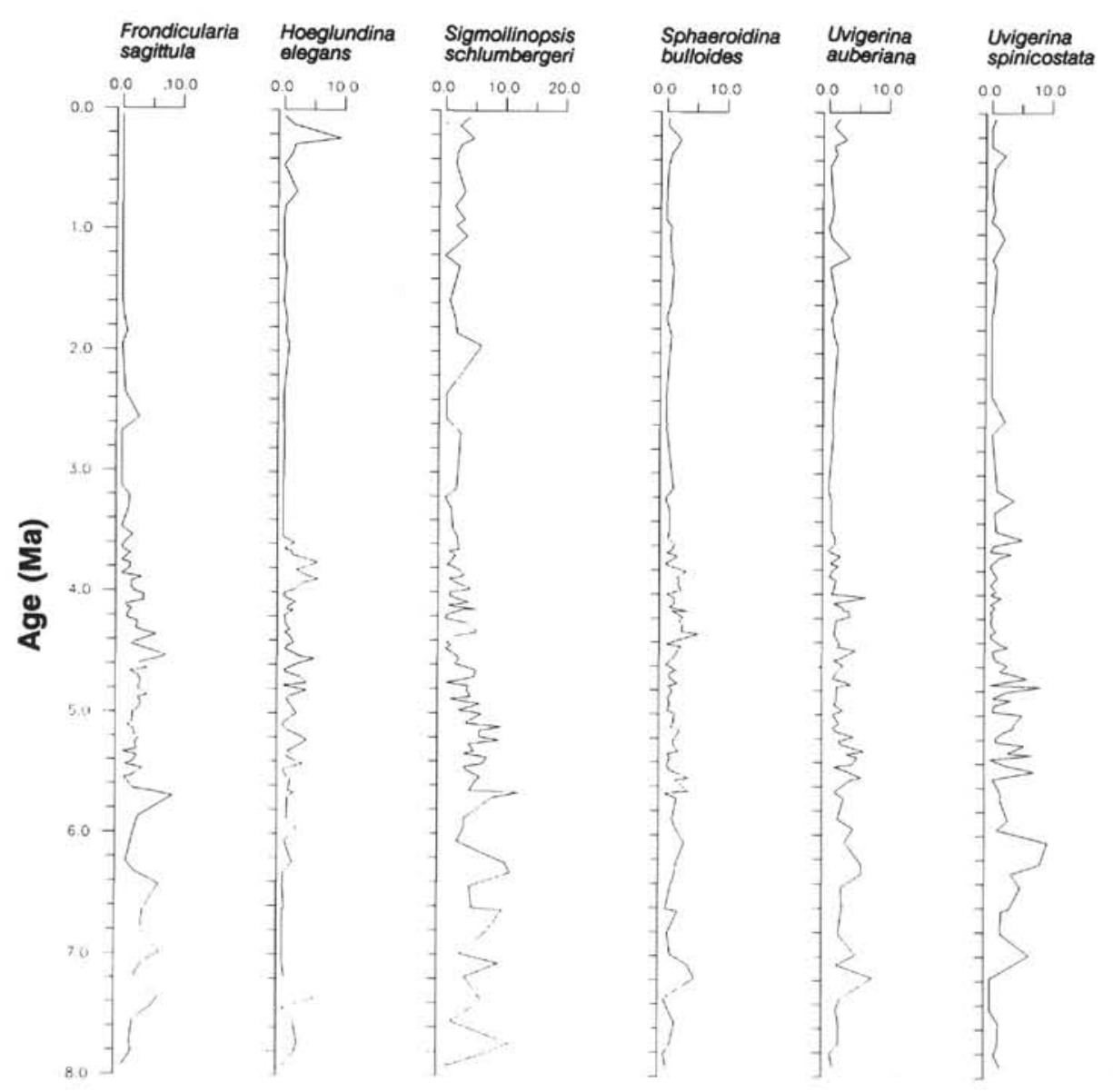

Figure 11. Relative abundance curves for benthic foraminiferal species belonging to the late Miocene/early Pliocene Assemblage of ODP Hole 728A. 

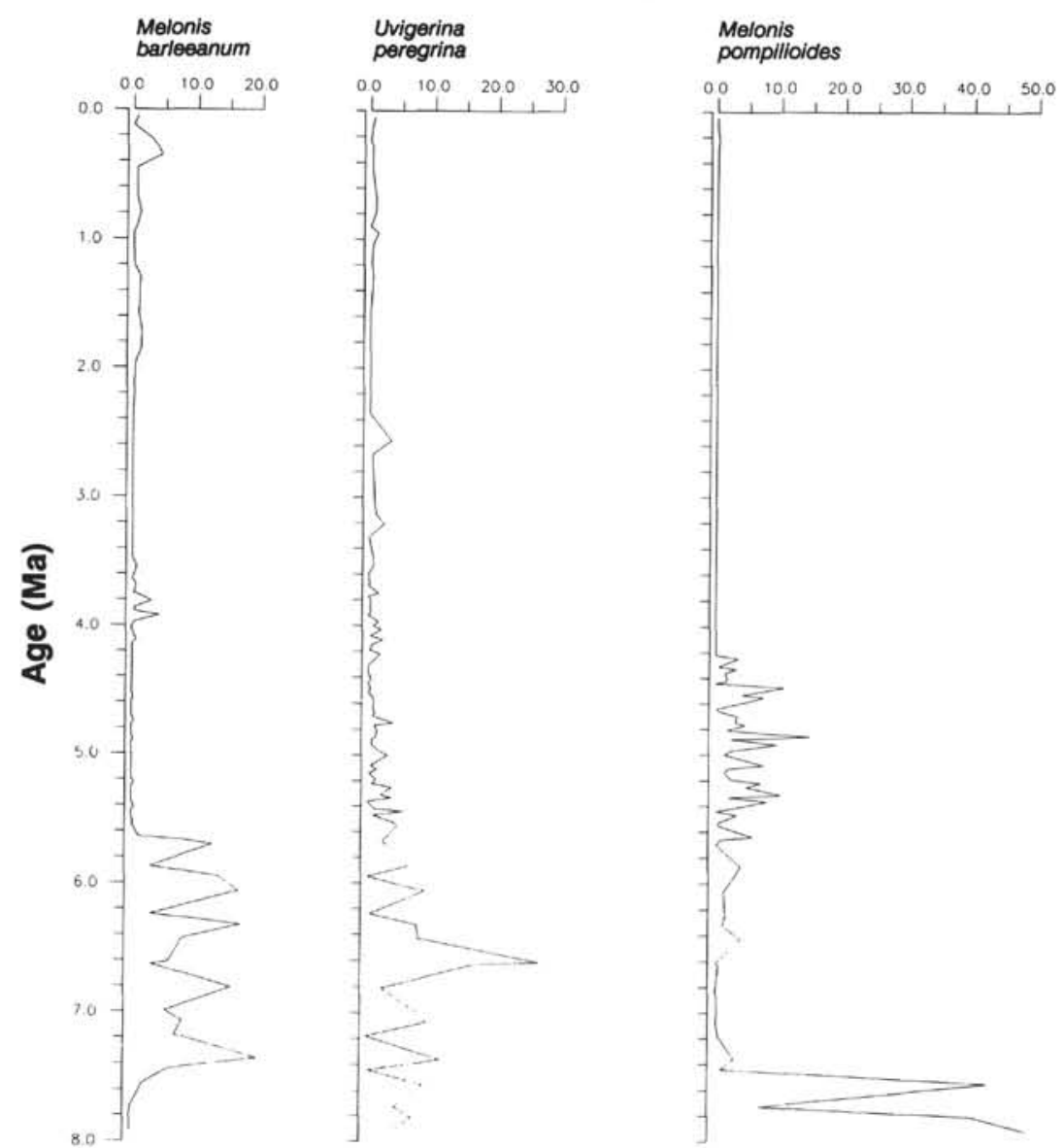

Figure 12. Relative abundance curves for benthic foraminiferal species belonging to the late Miocene Assemblage of ODP Hole 728A. 


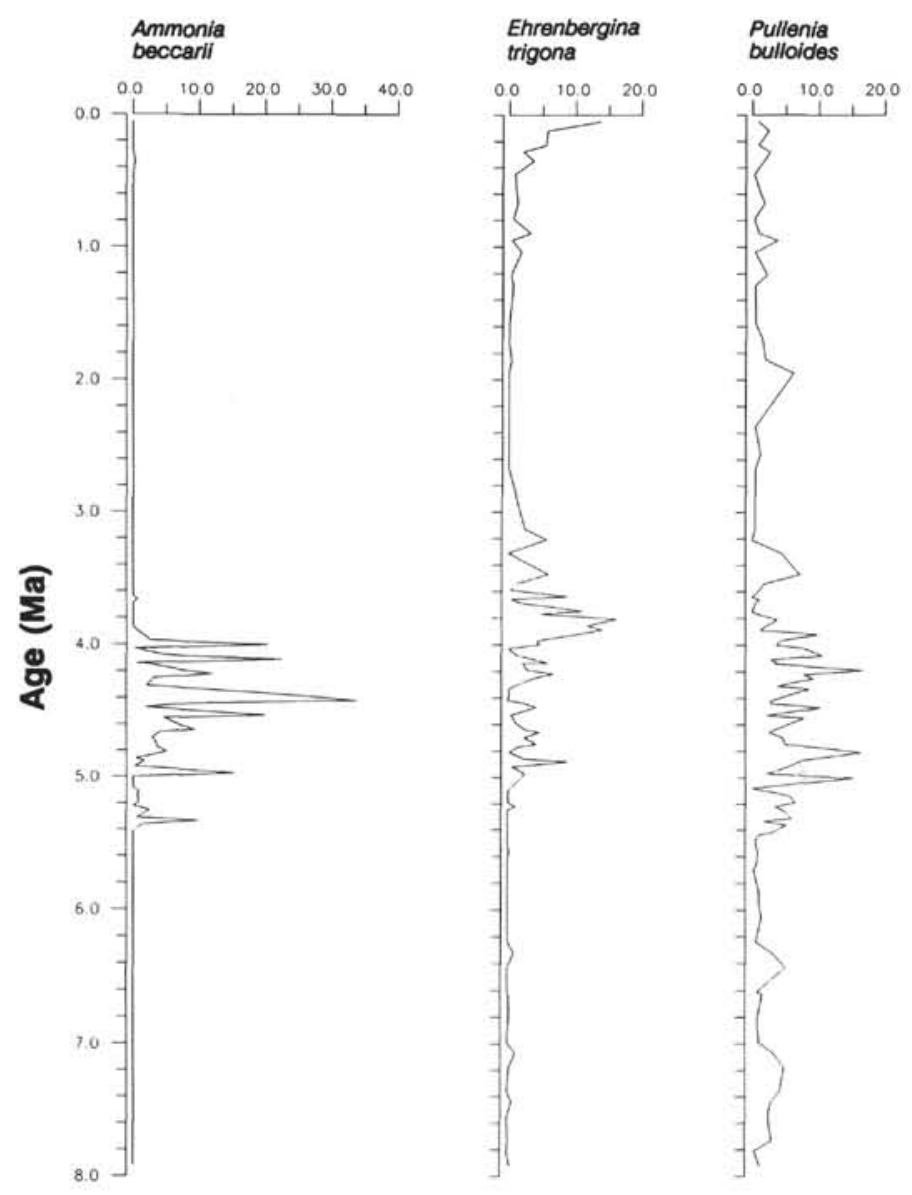

Figure 13. Relative abundance curves for benthic foraminiferal species belonging to the early Pliocene Assemblage of ODP Hole 728A. 

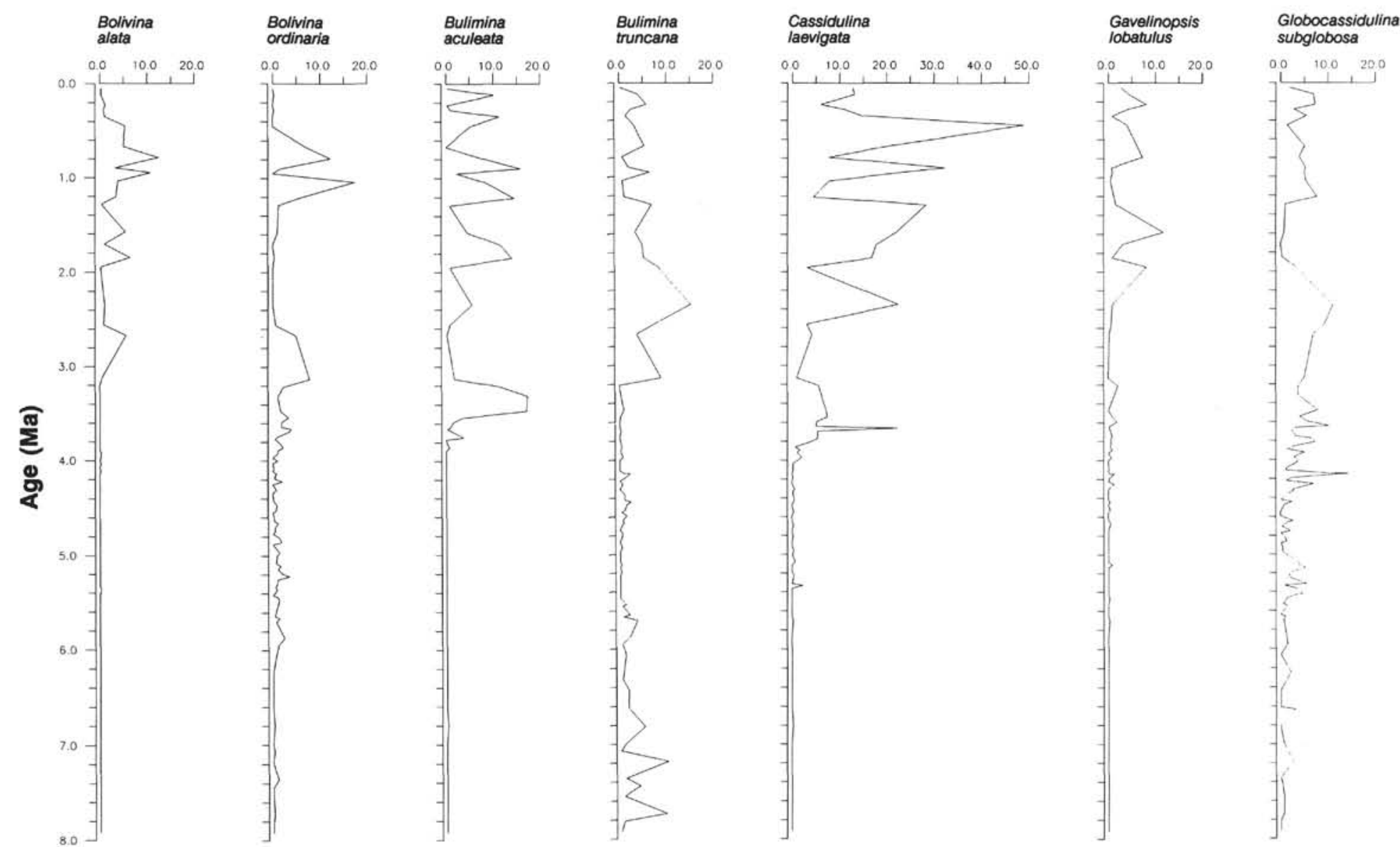

Hyalinea
balthica

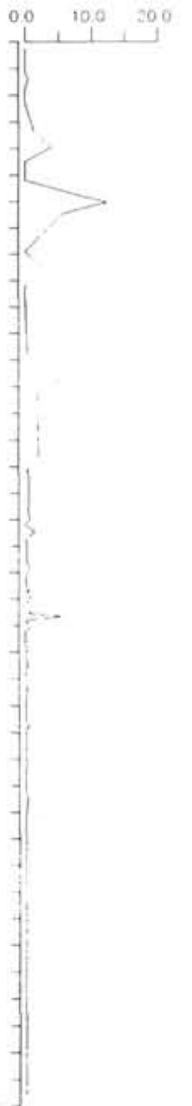

Figure 14. Relative abundance curves for benthic foraminiferal species belonging to the late Pliocene/Pleistocene Assemblage of ODP Hole 728A. 


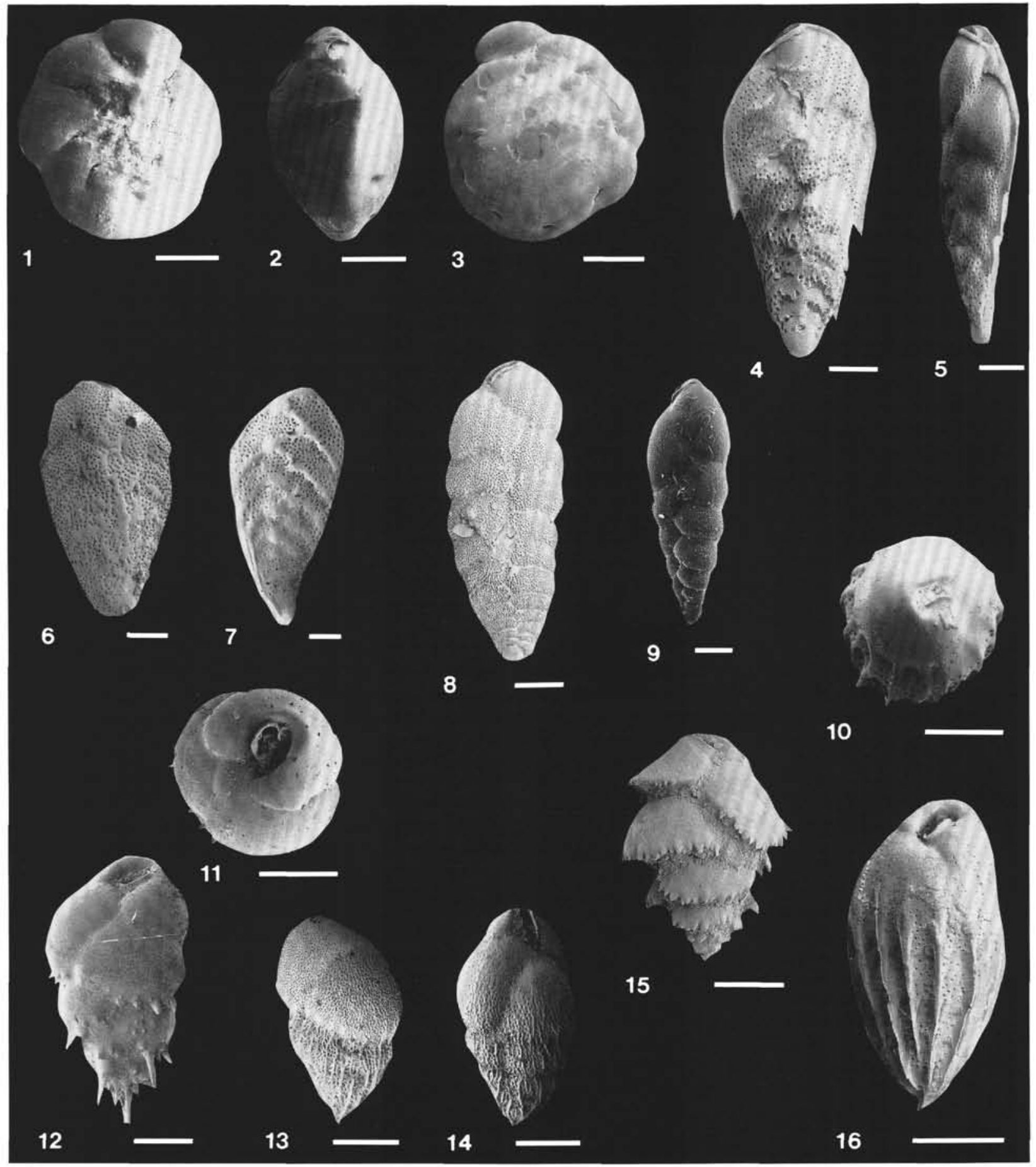

Plate 1. SEM micrographs. Scale bars equal $100 \mu \mathrm{m}$. 1-3. Ammonia beccarii (Linné), Section 117-728A-23X-CC. 4-5. Bolivina alata (Seguenza), Sample 117-728A-4H-4, 55-57 cm. 6-7. Bolivina ordinaria Phleger and Parker, Sample 117-726A-11X-4, 55-57 cm. 8-9. Bolivina seminuda Cushman, Sample 117-726A-6H-4, 55-57 cm. 10, 16. Bulimina truncana Guembel, 117-728A-8H-4, 55-57 cm. 11-12. Bulimina aculeata d'Orbigny, Sample 117-728A-1H-4, 55-57 cm. 13-14. Bulimina denudata Cushman and Parker, 117-725C-11X-2, 55-57 cm. 15. Bulimina marginata d'Orbigny, Sample 117-725C-11X-2, 55-57 cm. 


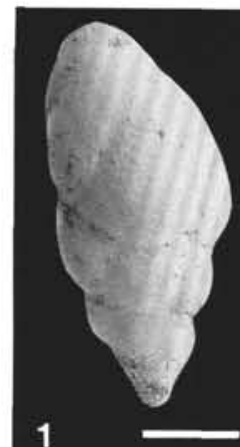

1

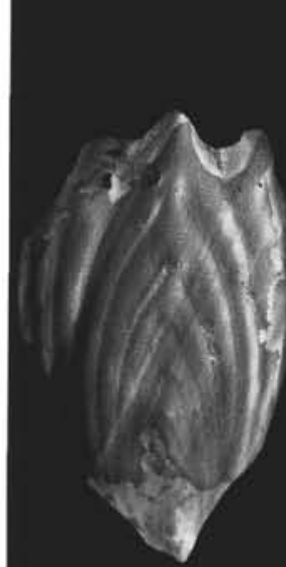

6

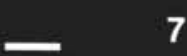

7
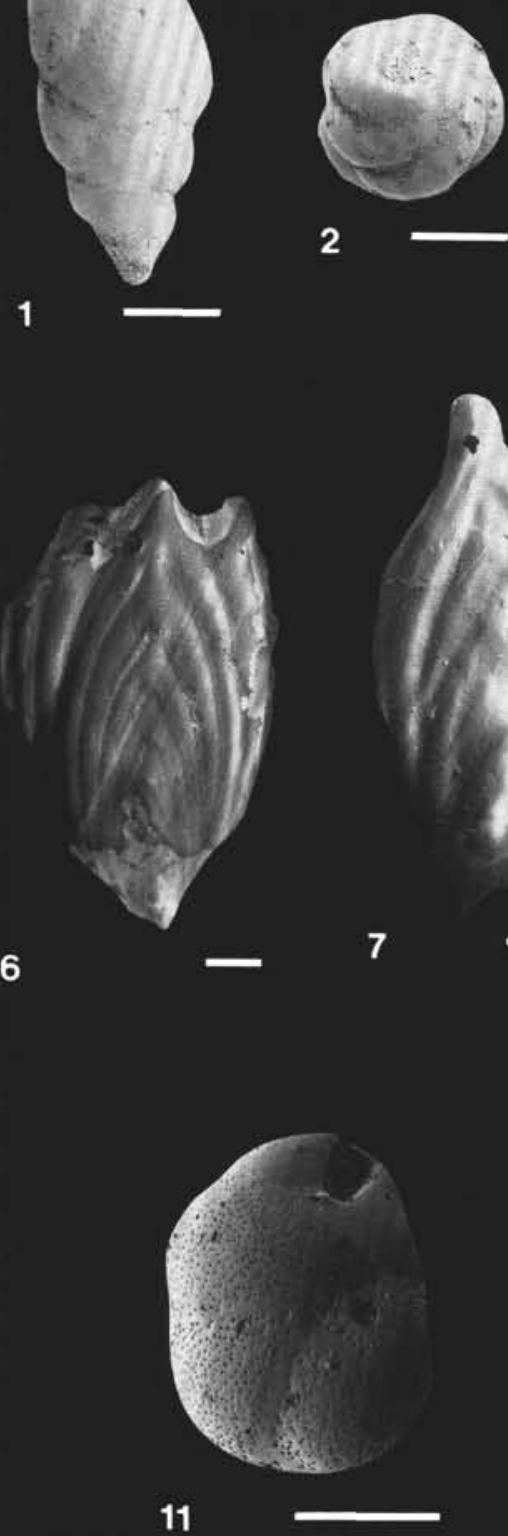

2

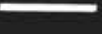

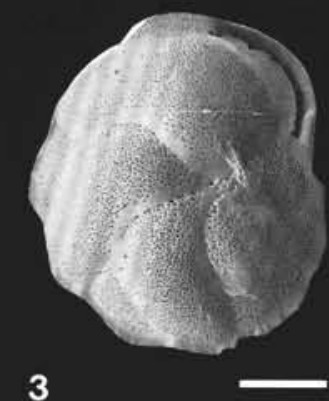

3
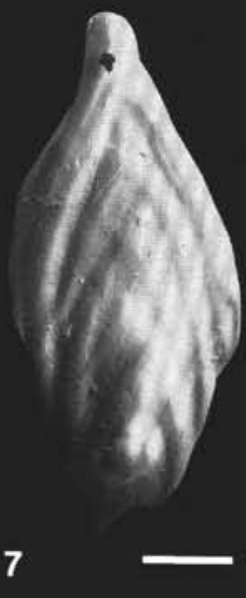

8
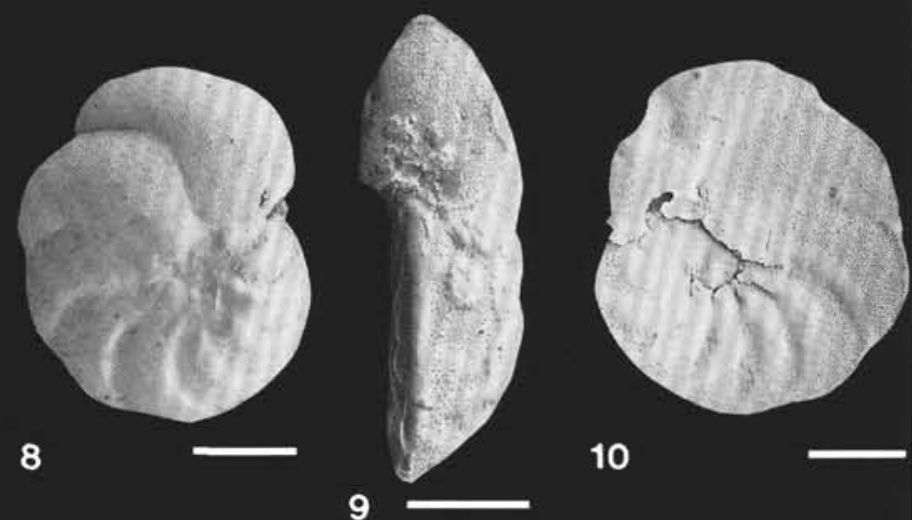

10

9
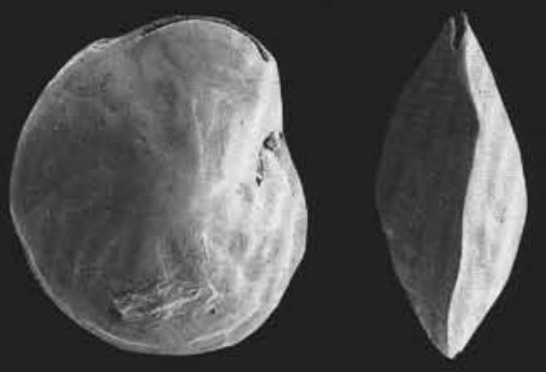

13

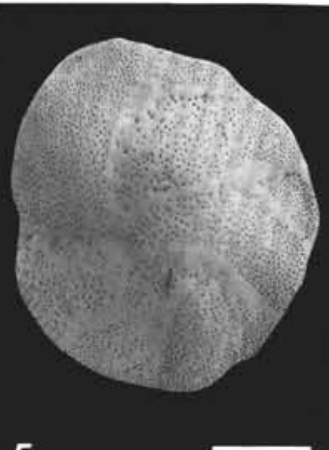

5
12
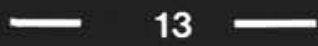

14

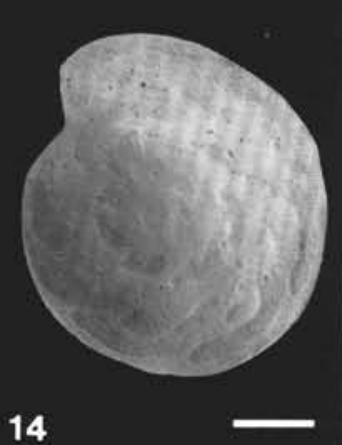

Plate 2. SEM micrographs. Scale bars equal $100 \mu \mathrm{m}$. 1-2. Buliminella elegantissima (d'Orbigny), Sample 117-728A-8H-4, 55-57 cm. 3-5. Cassidulina laevigata d'Orbigny, $117-728 \mathrm{~A}-2 \mathrm{H}-4,55-57 \mathrm{~cm}$. 6-7. Frondicularia sagittula van den Broeck, Section 117-728A-23X-CC. 8-10. Hanzawaia concentrica (Cushman), Sample 117-726A-2H-4, 55-57 cm. 11. Globocassidulina subglobosa (Brady), 117-728A-8H-4, 55-57 cm. 12-14. Hoeglundina elegans (d'Orbigny), Section 117-726A-1H-CC. 


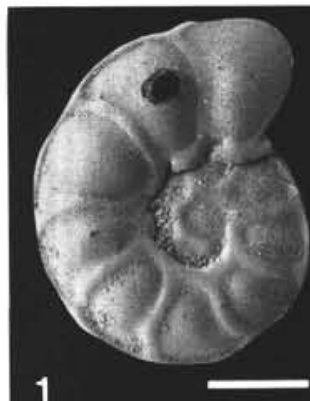

1

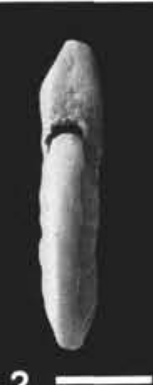

2

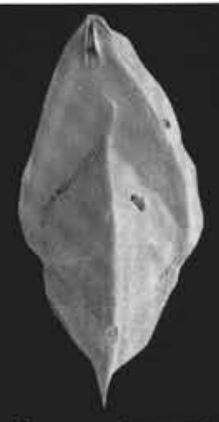

3
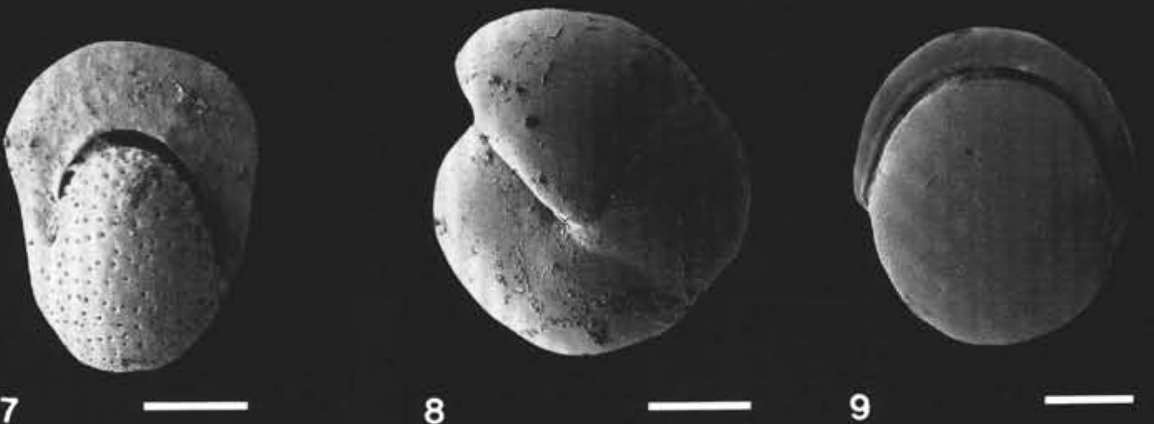

6
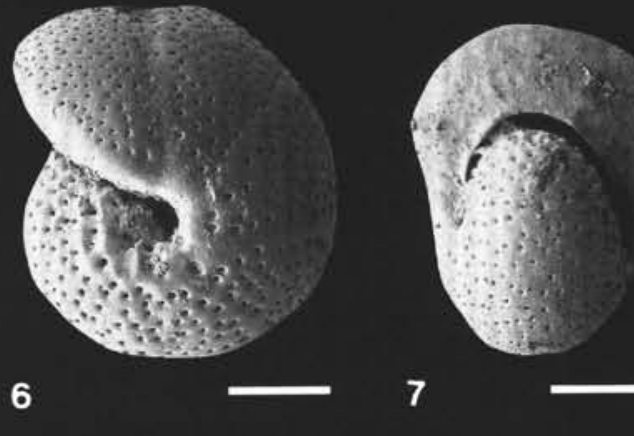

8

4
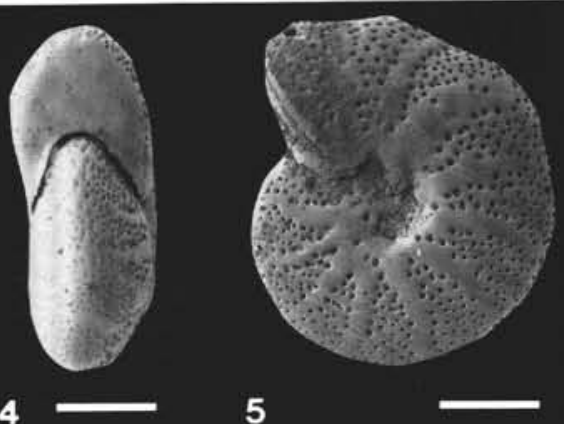

5

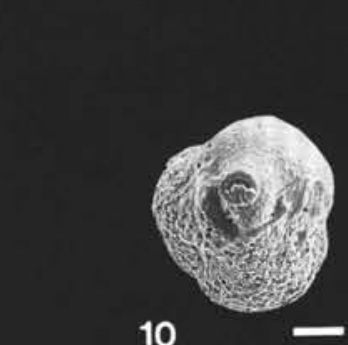

10

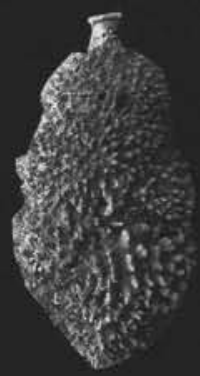

13

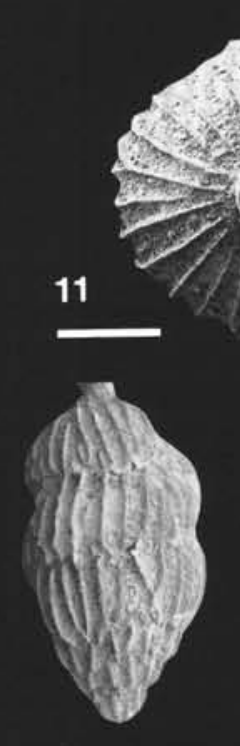

14

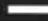

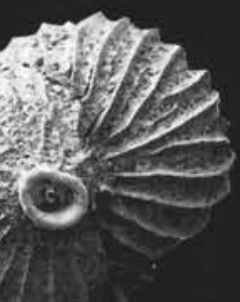

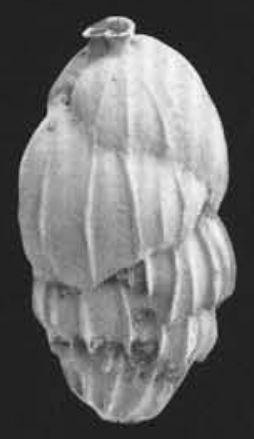

15

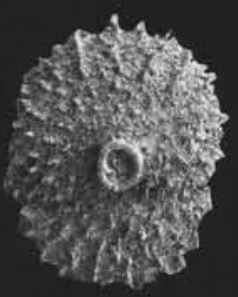

12

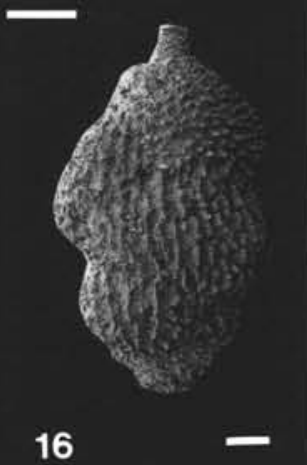

Plate 3. SEM micrographs. Scale bars equal $100 \mu \mathrm{m}$. 1-2. Hyalinea balthica (Schroeter), Sample 117-725C-6H-2, 55-57 cm. 3. Lenticulina iota (Cushman), Sample 117-725C-15X-4, 55-57 cm. 4-5. Melonis barleeanum (Williamson), Sample 117-728A-2H-4, 55-57 cm. 6-7. Melonis pompilioides (Fichtel and Moll), Sample 117-728A-36X-2, 55-57 cm. 8-9. Pullenia bulloides (d'Orbigny), Sample 117-728A-2H-4, 55-57 cm. 10, 13. Uvigerina auberiana d'Orbigny, Section 117-728-20X-CC. 11, 14-15. Uvigerina peregrina Cushman, Sample 117-728A-33X-1, 55-57 cm. $12,16$. Uvigerina spinicostata Cushman and Jarvis, Sample 117-728A-33X-1, 55-57 cm. 